\title{
Neuropilin-1 mediates lung tissue-specific control of ILC2 function in type 2 immunity
}

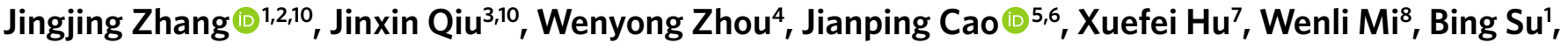 \\ Bin $\mathrm{He}{ }^{9 凶}$, Ju Qiu ${ }^{3}{ }^{\star}$ and Lei Shen ${ }^{1,2} \bowtie$
}

Group 2 innate lymphoid cells (ILC2s) are highly heterogeneous tissue-resident lymphocytes that regulate inflammation and tissue homeostasis in health and disease. However, how these cells integrate into the tissue microenvironment to perform tissue-specific functions is unclear. Here, we show neuropilin-1 (Nrp1), which is induced postnatally and sustained by lung-derived transforming growth factor beta-1 (TGFP1), is a tissue-specific marker of lung ILC2s. Genetic ablation or pharmacological inhibition of Nrp1 suppresses IL-5 and IL-13 production by ILC2s and protects mice from the development of pulmonary fibrosis. Mechanistically, TGF31-Nrp1 signaling enhances ILC2 function and type 2 immunity by upregulating IL-33 receptor ST2 expression. These findings identify Nrp1 as a tissue-specific regulator of lung-resident ILC2s and highlight Nrp1 as a potential therapeutic target for pulmonary fibrosis.

LC2s express transcription factor GATA3 and produce IL-5 and IL-13 in response to IL-33, IL-25, and thymic stromal lymphopoietin (TSLP $)^{1,2}$. They are considered tissue-resident lymphocytes and are especially enriched at barrier surfaces ${ }^{3}$. Within the local tissue, ILC2s not only establish a first-line defense system against infections, but also orchestrate tissue homeostatic functions through interactions with other tissue-resident cells, such as epithelial cells, stromal cells, and neurons ${ }^{4-7}$. Tissue-resident ILC2s are functionally heterogeneous in terms of their origins ${ }^{8}$. This is largely attributed to distinct transcriptional signatures acquired by tissue ILC2s under the influence of environmental cues, which endow ILC2s with expression of differential activating receptors and poised phenotypes under steady-state and inflammatory conditions $s^{9,10}$.

Studies have shown that ILC2s that are resident in different tissues including lung, adipose tissue, intestine, and skin, have distinct expression patterns of cytokine receptors, which consequently impact their cytokine production and therefore regulate the respective tissue-specific immunity ${ }^{11,12}$. The IL-33 receptor ST2 is expressed by almost all tissue ILC2s and is highly enriched in lung and adipose ILC2 $\mathrm{s}^{4}$. IL-33 is mainly produced by platelet-derived growth factor receptor alpha ${ }^{+}\left(\mathrm{PDGFR} \alpha^{+}\right)$stromal cells and preadipocytes in the adipose tissue, lung, and intestine ${ }^{13}$. Once engaged with ST2, IL-33 can strongly stimulate ILC2s proliferation and type 2 cytokines production. IL-33 signaling is also essential for generation of ILC2 memory ${ }^{14}$. The IL-25 receptor, IL-17RB, is highly expressed on ILC2s in the small intestine ${ }^{15}$. IL-25 is produced by specialized intestinal epithelial cells called tuft cells ${ }^{4,5}$. Upon IL-25 stimulation, ILC2s are activated and become inflammatory ILC2s that can secrete IL-17 in addition to IL-5 and IL-13. Inflammatory ILC2s are found in lung and mesenteric lymph nodes after worm infection or IL-25 injection ${ }^{16}$. By contrast, skin ILC2s express low levels of receptors for both IL-33 and IL-25, but highly express IL-18 receptor. IL-18 can activate skin ILC2s independent of IL-33 and IL-25 and has been demonstrated to influence activation of skin ILC2s in both the steady state and atopic dermatitis ${ }^{4}$. The diversity in receptor expression on ILC2s from different tissues ensures optimal ILC2 activation depending on the environmental need. Thus, identifying the tissue-specific features of ILC2s and how these features enable ILC2s to adapt to their tissue milieu to meet functional demands in the tissue microenvironment are the major challenges in the field.

Single-cell RNA sequencing (scRNA-seq) has been widely used to reveal tissue-specific signatures of tissue-resident immune cells ${ }^{17,18}$. Through mining the scRNA-seq data of tissue-resident ILC2s from both the public domain and our own group, we found that $\mathrm{Nrp1}$, the gene encoding Nrp1, is highly expressed in ILC2s from lung, but not in those from other tissue origins ${ }^{4,19}$. Nrpl is a transmembrane glycoprotein that acts as a co-receptor for extracellular ligands, including class 3 semaphorins (Sema3), isoforms of vascular endothelial growth factor (VEGF) and TGF $\beta$ (refs. ${ }^{20,21}$ ). Studies have shown that Nrp1 enhances the immunosuppressive function of natural regulatory $\mathrm{T}\left(\mathrm{T}_{\mathrm{reg}}\right)$ cells $\mathrm{s}^{22,23}$ and suppresses differentiation of tumor-infiltrated $\mathrm{CD}^{+}$memory $\mathrm{T}$ cells ${ }^{24,25}$. Hence, Nrp1 limits antitumor immune responses. Additionally, Nrp1 has been shown to be expressed by group 3 innate lymphoid cells (ILC3s) and to facilitate the migration of ILC3s in humans ${ }^{26}$. Beyond its role as an important regulator of immunity, whether Nrp1 is a tissue-specific

\footnotetext{
'Shanghai Institute of Immunology, Department of Immunology and Microbiology, and Key Laboratory of Cell Differentiation and Apoptosis of the Chinese Ministry of Education, Shanghai Jiao Tong University School of Medicine, Shanghai, China. ${ }^{2}$ Shanghai Key Laboratory of Tumor Microenvironment and Inflammation, Shanghai Jiao Tong University School of Medicine, Shanghai, China. ${ }^{3} \mathrm{CAS}$ Key Laboratory of Tissue Microenvironment and Tumor, Shanghai Institute of Nutrition and Health, University of Chinese Academy of Sciences, Chinese Academy of Sciences, Shanghai, China. ${ }^{4}$ Department of Thoracic Surgery, Shanghai Chest Hospital, Shanghai Jiao Tong University, Shanghai, China. ${ }^{5} \mathrm{NHC}$ Key Laboratory of Parasite and Vector Biology, National Institute of Parasitic Diseases, Chinese Center for Disease Control and Prevention, Shanghai, China. ${ }^{6}$ The School of Global Health, Chinese Center for Tropical Diseases Research, Shanghai Jiao Tong University School of Medicine, Shanghai, China. ${ }^{7}$ Department of Thoracic Surgery, Shanghai Pulmonary Hospital, Tongji University School of Medicine, Shanghai, China. ${ }^{8}$ Department of Integrative Medicine and Neurobiology, School of Basic Medical Science; Institutes of Integrative Medicine, Shanghai Medical College, Fudan University, Shanghai, China. 'Department of Critical Care Medicine, Shanghai Chest Hospital, Shanghai Jiao Tong University, Shanghai, China. ${ }^{10}$ These authors contributed equally Jingjing Zhang, Jinxin Qiu.
}

凶e-mail: bin_he@sjtu.edu.cn; qiuju@sibs.ac.cn; Ishen@shsmu.edu.cn 
feature of lung ILC2s, why it is expressed in lung ILC2s, and what the role of Nrp1 is in ILC2 and lung homeostasis remain unknown.

Here, using the high-parameter flow cytometry FACSymphony to screen the tissue-specific signatures at the protein level, we show that Nrp1 is distinctively expressed by lung ILC2s. Furthermore, we identify TGF $\beta 1$ from lung tissue as an important environmental signal to induce and sustain $\mathrm{Nrp} 1$ expression by lung ILC2s. Functionally, Nrp1 signaling enhances ILC2 activation in a cell-intrinsic manner. Ablation of Nrp1 specifically in ILC2s decreases type 2 cytokine production and protects mice from pulmonary fibrosis. These results indicate that Nrp1 is a potential therapeutic target for pulmonary fibrosis and provide direction for the improvement of therapeutic strategies to target tissue-specific pathology.

\section{Results}

Nrp1 is a tissue-specific marker of lung ILC2s. Studies have characterized the tissue heterogeneity of ILC2s at the transcriptomic level ${ }^{4,19}$. Through mining scRNA-seq data, we found that lung ILC2s highly expressed Nrp1 in both mice and humans (Fig. 1a and Extended Data Fig. 1a). To validate Nrp1 expression on lung ILC2s at the protein level, we used high-parameter flow cytometry to analyze ILC2s from lung, pancreas, large intestine, and small intestine. ILC2s were defined as $\operatorname{lin}^{-}\left(\mathrm{CD} 3^{-} \mathrm{B} 220^{-} \mathrm{CD} 5^{-} \mathrm{CD} 11 \mathrm{~b}^{-} \mathrm{CD} 11 \mathrm{c}^{-}\right)$ $\mathrm{CD} 5^{+} \mathrm{CD} 127^{+} \mathrm{GATA}^{+}$live cells (Extended Data Fig. 1b,c). $t$-distributed stochastic neighbor embedding ( $t$-SNE) analysis revealed that ILC2s from the same tissue were clustered together, and cells from different tissues formed separate clusters, suggesting that ILC2s exhibit striking cross-tissue heterogeneity (Fig. 1b). A heatmap of ten ILC2-related markers clearly separated ILC2s from different tissues on the basis of their expression levels. Pancreas ILC2s were characterized by high expression of CD25, MHC II, and ST2 (Fig. 1c). Lung ILC2s highly expressed CCR9, ST2, and Nrp1 (Fig. 1c). Large-intestine ILC2s were featured by enriched expression of PD-1 and CD69 (Fig. 1c). Small-intestine ILC2s highly expressed CCR9, IL-17RB, KLRG1, and SCA1 (Fig. 1c). To visualize the tissue expression landscape of ILC2s, feature plots were displayed for each tissue-specific marker. Most of the tissue-specific markers, including CD69, IL-17RB, KLRG1, SCA1, CD25, ST2, and Nrp1, were expressed in the vast majority of ILC2s, but PD-1, CCR9, and MHC II were strongly expressed only in a small subset of ILC2s from large intestine, lung, and pancreas, respectively (Fig. 1d).

Remarkably, Nrp1 stood out as a tissue-specific marker of lung ILC2s, whereas ILC2s from other tissues expressed marginal to undetectable levels of Nrp1 (Fig. 1d-g). Nrp1 was expressed by around $80 \%$ of lung ILC2s (Fig. 1g,h), and almost all Nrp1 $1^{+}$ILC2s co-expressed pan-ILC2 markers ST2, CD25, and SCA1 (Fig. 1h). In addition to mature, tissue-resident ILC2s, we also analyzed whether ILC2 precursors in bone marrow expressed Nrp1. As expected, Nrp1 was not expressed on ILC2 precursors (Fig. 1i). The expression of Nrp1 by lung ILC2s was also confirmed in mice with different genetic backgrounds in addition to those with the C57BL/6 background (Fig. 1j). Together, we conclude that Nrp1 is a tissue-specific feature of lung ILC2s. To rule out the possibility that the adaptive immune system affects Nrp1 expression in lung

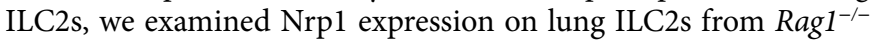
mice, which lack $\mathrm{T}$ and $\mathrm{B}$ cells. In accordance, with the observation in wild-type mice, lung ILC2s from Rag1 ${ }^{-/-}$mice highly expressed Nrp1 compared with those from the other tissues (Fig. 1k). These data suggest that Nrp1 expression in lung ILC2s is independent of the adaptive immune compartment.

Lung-derived signals sustain Nrp1 expression by ILC2s. To address when ILC2s acquire the Nrp1 signature in lung tissue, we delineated the temporal kinetics of Nrp1 expression on ILC2s from various tissues throughout ontogeny. Intriguingly, ILC2s expressed little or no Nrp1 in all the tissues from newborn mice (Day 1) (Fig. $2 a, b)$. The expression of Nrp1 was gradually increased in lung ILC2s with the development of mice and reached $80 \%$ of ILC2s in the adult stage (Fig. 2a,b). However, Nrp1 expression on ILC2s from other tissues remained marginal throughout the period of observation (Fig. 2a,b). This raised the possibility that Nrp1 expression is dependent on signals from the lung tissue microenvironment. To test this hypothesis, we transferred ILC2s isolated from either lung or intestine into immune-deficient $\mathrm{Rag}^{-/-} \mathrm{Il}_{2 \mathrm{rg}} \mathrm{r-}^{-/}$hosts, in which ILC2s from different tissues were analyzed for their Nrp1 expression level 3 weeks later. Lung-derived ILC2s downregulated the Nrp1 expression level dramatically upon reconstitution in the intestine, including both large and small intestine (Fig. 2c-f). By contrast, ILC2s isolated from intestine significantly upregulated Nrp1 expression following transfer to lung (Fig. $2 \mathrm{c}-\mathrm{f}$ ). Together, these data suggest that Nrp1 expression by ILC2s could be induced by lung environment and suppressed by the intestine environment in vivo.

Next, the adaption of tissue ILC2s to the environmental cues was investigated in vitro by co-culture with isolated mononuclear cells from different tissues identified with separate congenic leukocyte markers (Fig. 2g-i). We found that intestinal cells efficiently suppressed Nrp1 expression on lung ILC2s (Fig. 2g). Conversely, lung cells promptly shaped intestinal ILC2s to express Nrp1 within 24 hours of culture (Fig. 2h,i). The co-culture system enabled simulation of the rapid kinetics of ILC2 migration to tissue parenchyma in vivo and their tissue adaptability. Taken together, these data demonstrate that lung tissue signals are required to induce and maintain Nrp1 expression in ILC2s.

TGFß1 induces expression of Nrp1 by lung ILC2s. We next sought to identify the molecular signals in the lung environment that induce Nrp1 expression on ILC2s. Given that Nrp1 is a co-receptor for several growth factors, including Sema3a, VEGF $\alpha$, and TGF $\beta 1$, we reasoned that these ligands might potentiate the expression of Nrp1 on lung ILC2s. We first examined the expression levels of TGF $\beta 1$, VEGF $\alpha$, and Sema3a by quantitative RT-PCR (qRT-PCR) in the lung tissue. Compared with other tissues, lung had the highest expression level for all of these ligands (Fig. 3a-c). To further define the key environmental signals in the lung that support the expression of Nrp1, Nrp1-negative ILC2s were treated with TGF $\beta 1$,

Fig. 1 | Nrp1 is a tissue-specific marker of lung ILC2s. a, Dot plot shows the abundance and intensity of Nrp1 expression in various tissues. scRNA-seq data of mouse ILC2s were obtained from Gene Expression Omnibus (GEO) under accession code GSE117568. BM, bone marrow. b-h, Mononuclear cells isolated from lung $(\mathrm{L})$, pancreas (P), large intestine (LI), and small intestine (SI) of C57BL/6 mice were stained with a 15-color ILC panel and analyzed by FACSymphonyA3. b, $t$-SNE was performed after concatenating ILC2s (lin-CD45+CD127+ST2+CD $\left.25^{+}\right)$from different tissues by FlowJo VX. c, A heatmap analysis based on mean fluorescence intensity (MFI) of ten markers is displayed for various tissue-resident ILC2s. $\mathbf{d}, t-S N E$ feature plots are shown to highlight the expression level of ten markers. e, The expression level of Nrp1 on ILC2s from various tissues. f, MFI of Nrp1 on ILC2s from various tissues ( $n=5 /$ group). g, Frequency of $\mathrm{Nrp1}^{+}$ILC2s in different tissues ( $n=5 /$ group). $\mathbf{h}$, Flow cytometry was used to analyze lung ILC2s for the expression level of Nrp1, ST2, CD25, SCA1, KLRG1, and CCR9. i, Nrp1 expression was analyzed on BM ILC2 precursors (lin-CD45+CD127+ST2+CD25+) by flow cytometry. j, Frequency of $\mathrm{Nrp1}^{+}$ILC2s in lungs from mice with different genetic backgrounds ( $n=4 /$ group). k, Frequency of Nrp1+ ILC2s in P, L, LI, and SI from Rag1-/mice ( $n=4$ /group). Each symbol represents an individual mouse $(\mathbf{f}, \mathbf{g}, \mathbf{j}, \mathbf{k})$. Data are representative of three independent experiments and are presented as mean \pm s.e.m. For statistical analysis in $\mathbf{f}, \mathbf{g}$, and $\mathbf{k}$, a one-way analysis of variance (ANOVA) was used. 
VEGF164, Sema3a, and the ILC2-activating cytokine IL-33. Among these factors, TGF $\beta 1$ and IL-33 alone induced Nrp1 expression on ILC2s (Fig. 3d,e), but TGF $\beta 1$ exhibited a much stronger effect than did IL-33 (Fig. 3d,e). The messenger-RNA (mRNA) expression level of Nrp1 was also increased significantly in TGF $\beta 1$-treated ILC2s, indicating that Nrp1 expression in ILC2s is regulated at the transcriptional level by TGF $\beta 1$ (Extended Data Fig. 1d). Furthermore, TGF $\beta 1$ was shown to induce Nrp1 expression in a dose-dependent manner (Fig. 3f,g). Importantly, the effect of TGF $\beta 1$ on Nrp1 induction was observed in human ILC2s as well, suggesting a

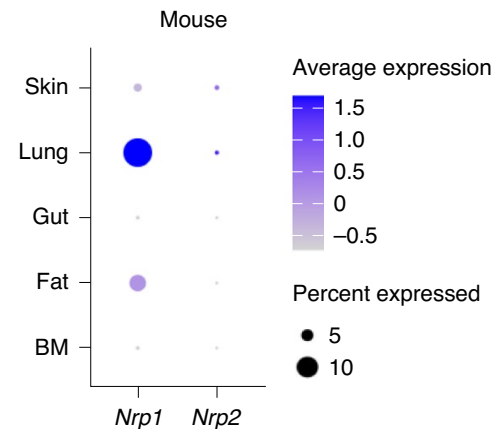

b

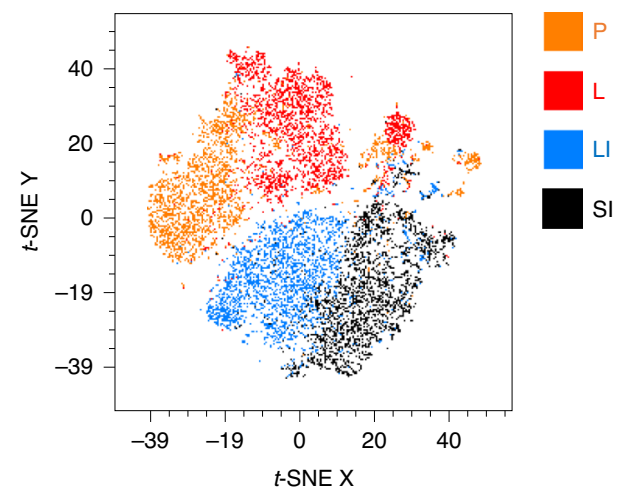

d
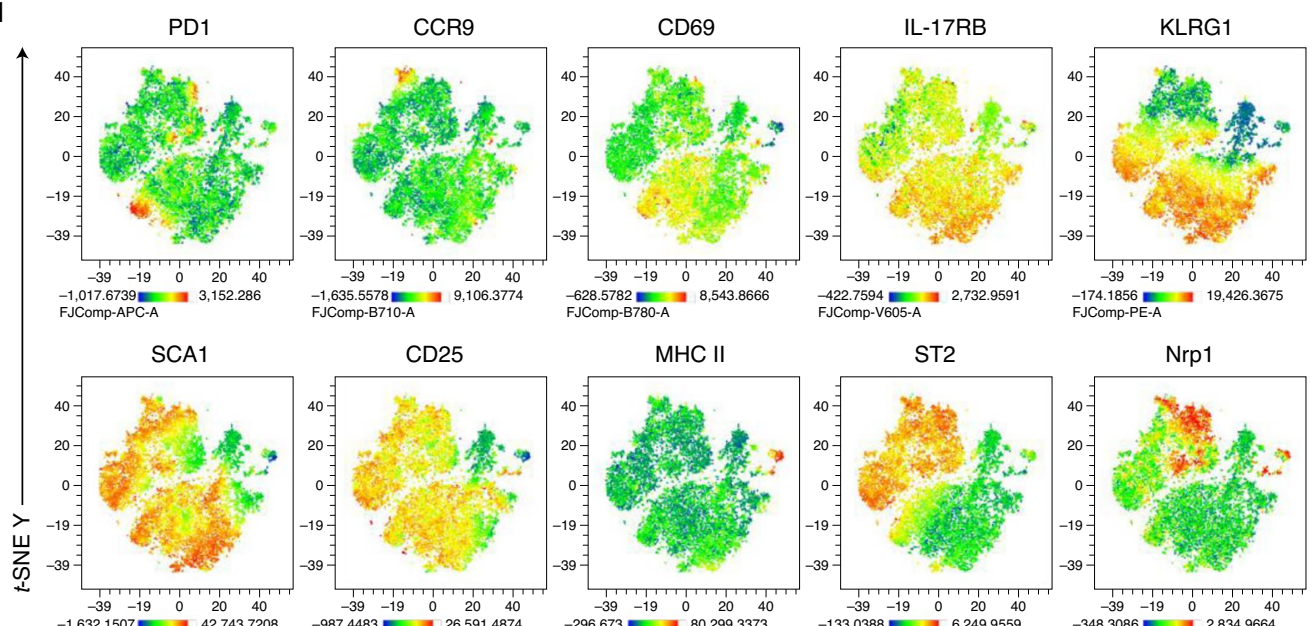

$\begin{array}{ccccc}-39 & -19 & 0 & 20 & 40 \\ -1,632.1507 & & 42,743.7208\end{array}$ FJComp-V650-A

$t$-SNE X
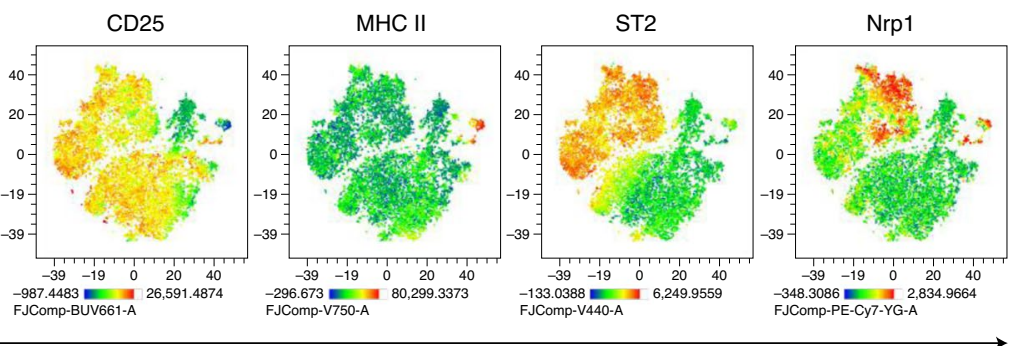

h
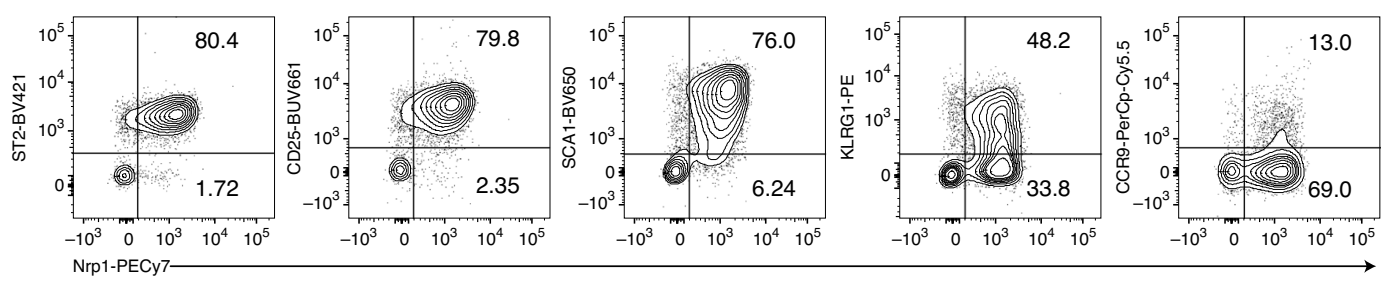
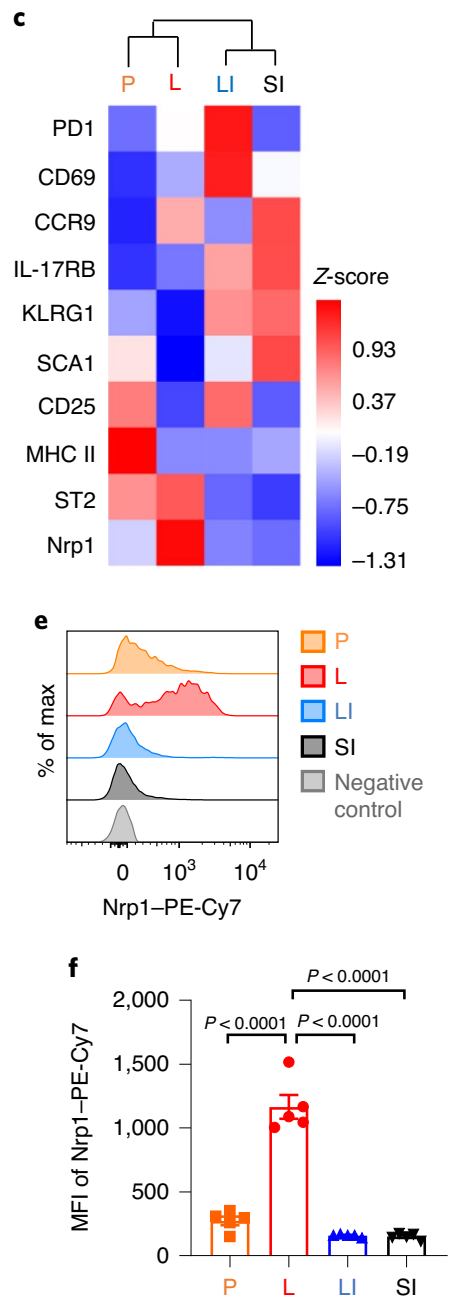

g

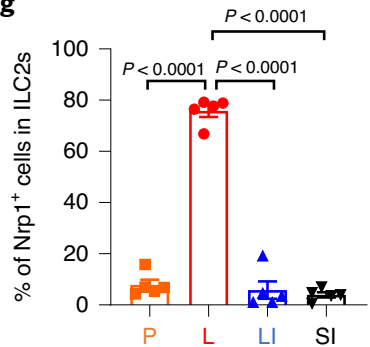

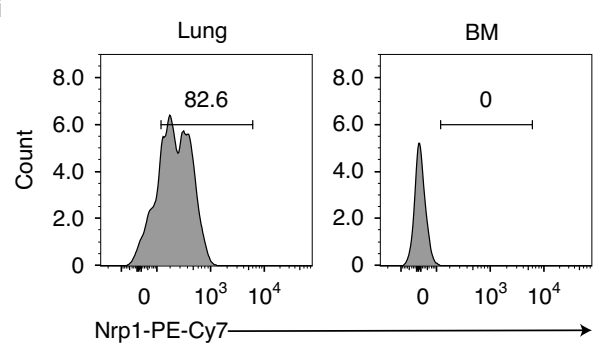

j

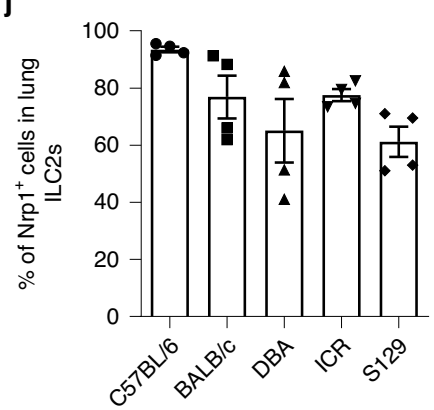

k

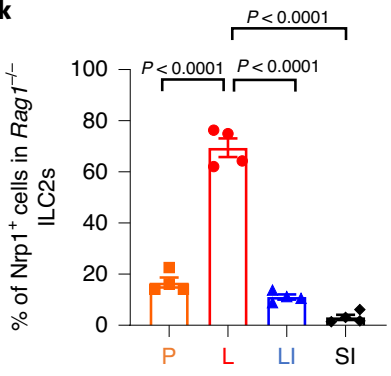


a

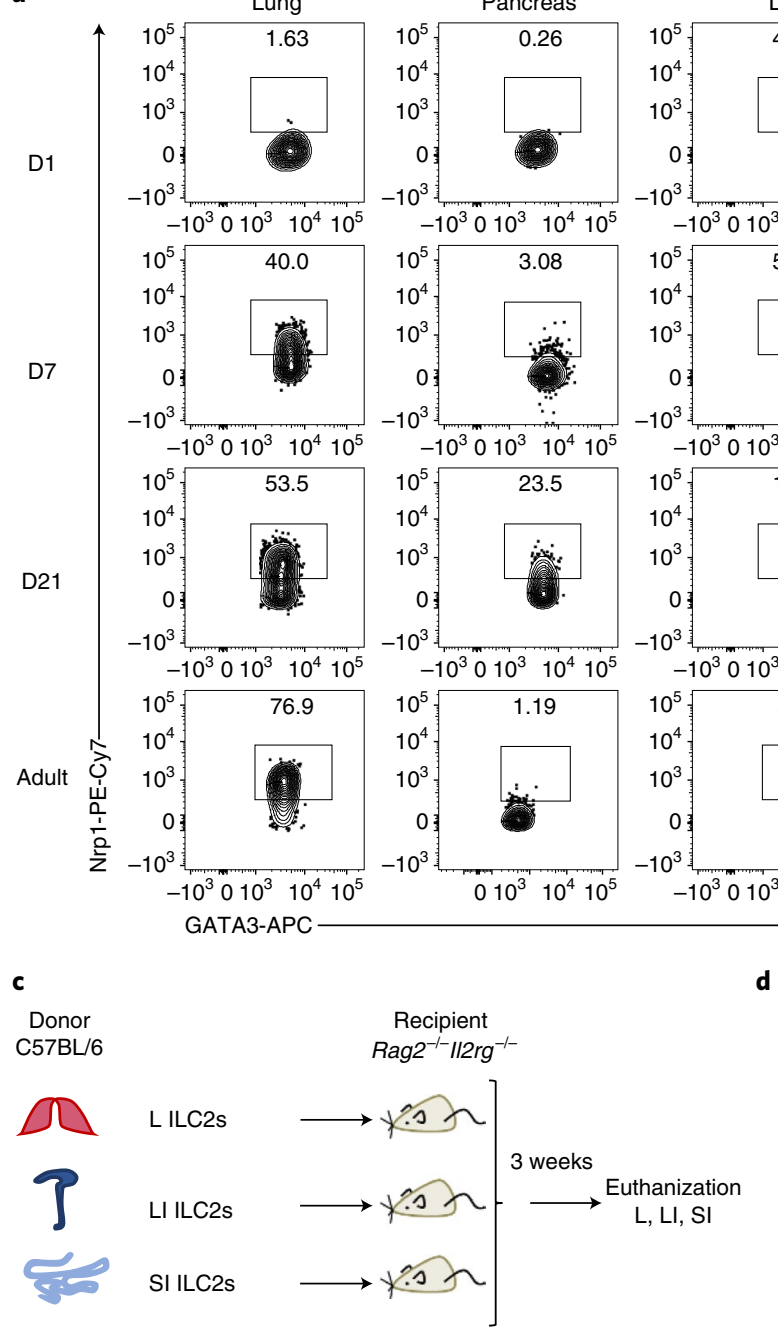

e

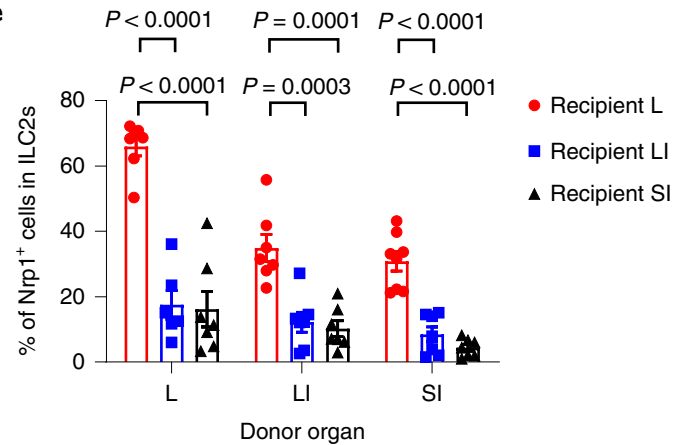

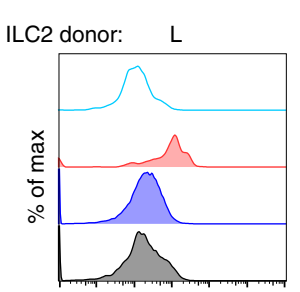
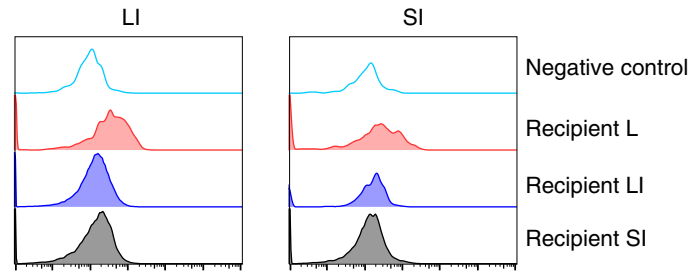

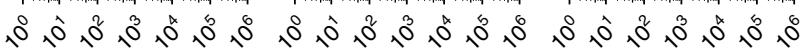
Nrp1-PE-Cy7
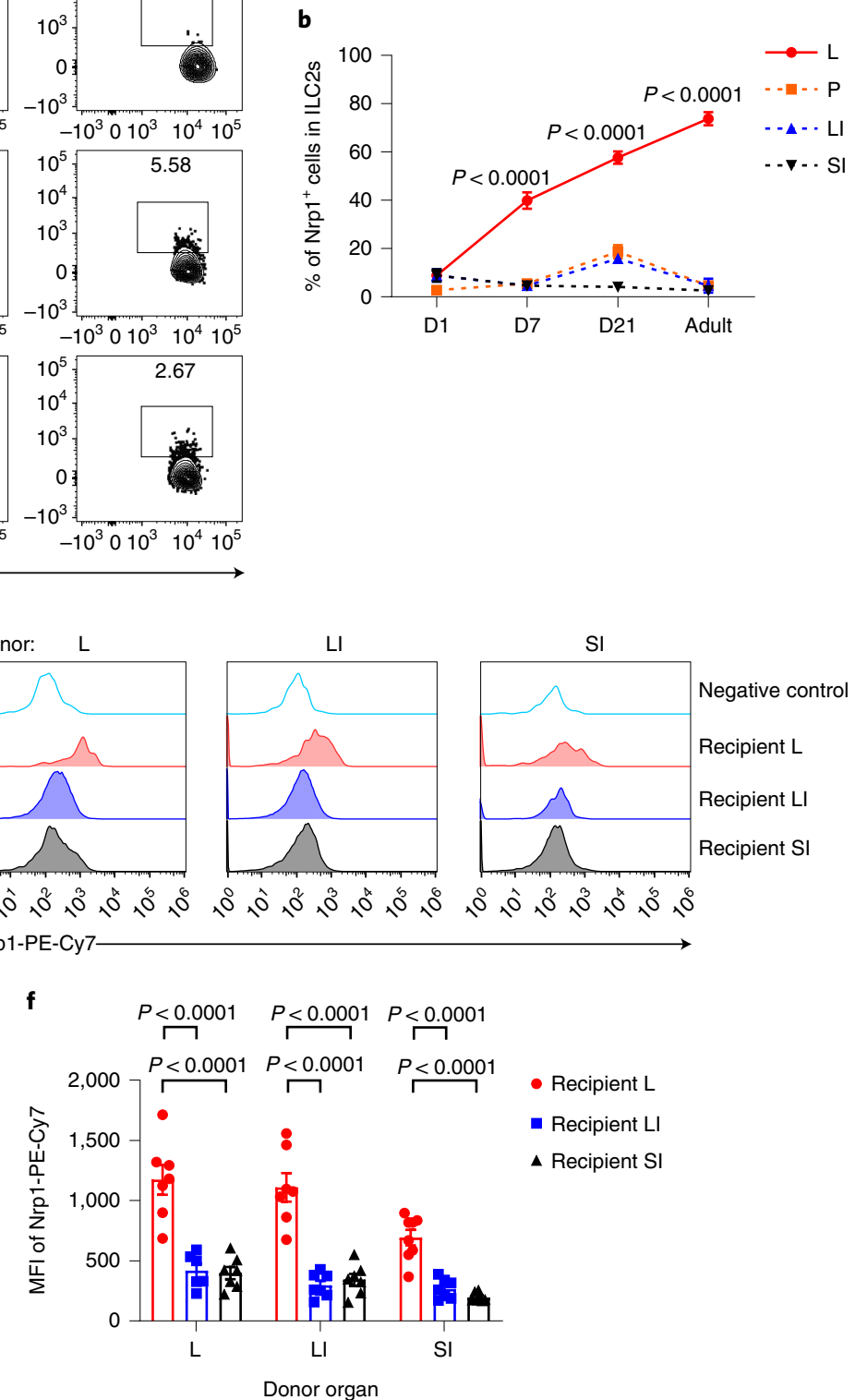

$\mathbf{g}$

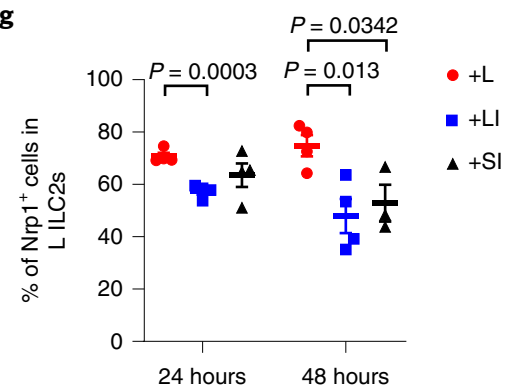

h

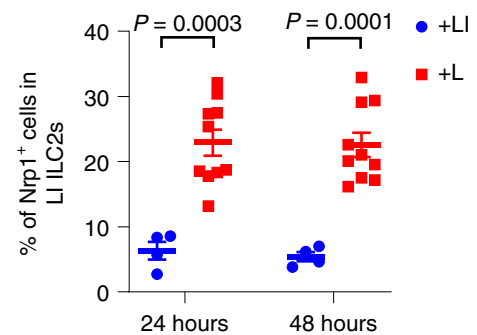

i

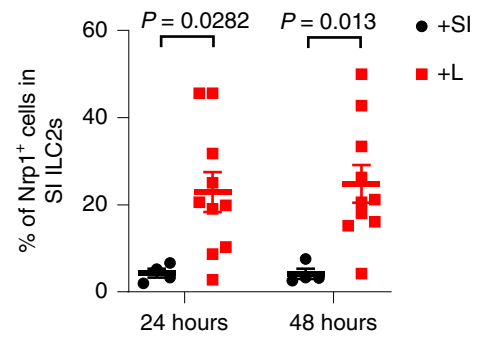

species-conserved regulation of Nrp1 by TGF $\beta 1$ (Fig. 3h). Because the expression level of TGF $\beta$ RII on large-intestine ILC2s was similar to that in lung ILC2s (Fig. 3i), we therefore reasoned that the enhanced Nrp1 expression in lung ILC2s could be attributed to high levels of TGF $\beta 1$. Indeed, lung had the highest level of bioactive TGF $\beta 1$ compared with the levels in other tissues (Fig. 3j). To 
Fig. 2 | Lung-derived signals are required for Nrp1 expression by ILC2s. a,b, Nrp1 expression level on ILC2s from various tissues at the indicated time points. Cells were gated on live ILC2s (lin $\left.{ }^{-} \mathrm{CD}_{4} 5^{+} \mathrm{CD} 127^{+} \mathrm{ST} 2^{+} \mathrm{CD} 25^{+}\right)(n=10,3,8,8$ for D1; $n=11,4,8,8$ for D7; $n=7,6,7,7$ for D21; $n=6,4,6,7$ for

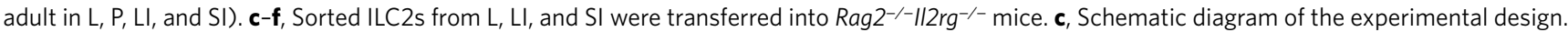
d, Representative expression level of Nrp1 on ILC2s from different tissues. e,f, Percentage of Nrp1+ ILC2s and MFI of Nrp1 on ILC2s in different tissues

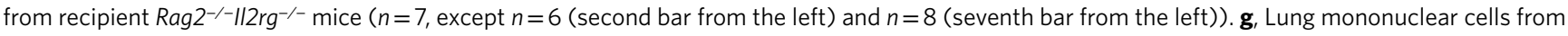
CD45.2 mice were co-cultured with lamina propria lymphocytes (LPLs) of LI and SI or L mononuclear cells from CD45.1 mice for 24 hours or 48 hours. The frequency of CD45.2+ $\mathrm{Nrp1}^{+}$ILC2s was analyzed ( $n=4$ except $n=3$ (first column from right)). $\mathbf{h}$, LPLs of LI from CD45.2 mice were co-cultured with $\mathrm{L}$ mononuclear cells from CD45.1 mice for 24 hours or 48 hours. The frequency of CD $45.2^{+} \mathrm{Nrp1}^{+} \mathrm{ILC} 2 \mathrm{~s}$ was analyzed $(n=4$ for the blue groups, $n=10$ for the red groups). $\mathbf{i}$, LPLs of SI from CD45.2 mice were co-cultured with L mononuclear cells from CD45.1 mice for 24 hours and 48 hours. The frequency of CD45. $2^{+} \mathrm{Nrp1}^{+}$ILC2s was detected ( $n=4$ for the black groups, $n=10$ for the red groups). Data are representative of 3-8 independent experiments and are presented as mean \pm s.e.m. For statistical analysis, the following tests were used. b,e,f, One-way ANOVA. g-i, Two-tailed unpaired Student's $t$ test.

test whether TGF $\beta 1$ plays a role in maintaining Nrp1 expression on lung ILC2s, anti-TGF $\beta 1$ blocking antibody was added to the co-culture system in vitro. Blockade of TGF $\beta 1$ reduced the frequency of $\mathrm{Nrp}^{+}$ILC2s in the culture of lung mononuclear cells (Fig. 3k). TGF $\beta 1$-blocking antibody also abolished the increase of Nrp1 expression on intestine ILC2s induced by lung mononuclear cells (Fig. 31,m). Collectively, these results identify TGF $\beta 1$ as a lung-derived signal that drives and maintains Nrp1 expression.

TGF $\beta 1$ has been reported to signal through a heterodimeric receptor in the plasma membrane consisting of TGF- $\beta$ receptor II (TGF $\beta$ RII) and TGF $\beta \mathrm{RI}^{27}$. LY2109761 and SB431542, specific inhibitors of TGF $\beta$ RII/TGF $\beta$ RI and TGF $\beta$ RI downstream signaling pathways, abolished Nrp1 induction by TGF $\beta 1$ in Nrp1-negative ILC2s sorted from large intestine (Fig. 3n). Meanwhile, phosphorylation of Smad2/Smad3, crucial mediators of the TGF $\beta$ RII/TGF $\beta$ RI downstream cascade, was increased in ILC2s treated with TGF $\beta 1$ (Fig. 3o). SIS3 HCl, a specific inhibitor of Smad3 phosphorylation, significantly suppressed the induction of Nrp1 by TGF $\beta 1$ (Fig. 3p). These data collectively suggest that TGF $\beta$ RII/TGF $\beta$ RI signaling is required for Nrp1 induction by TGF $\beta 1$.

Nrp1 ablation in ILC2s prevents development of fibrosis. To address whether Nrp1 plays a role in regulating the function of lung ILC2s, we generated $I d 2^{\text {cre-ERT2 }} N r p 1^{\text {f/f }}$ mice to selectively ablate Nrpl expression in the ILC lineage (Id $2^{\text {cre-ERT2 }} N r p 1^{\mathrm{f} /+}$ as WT, $I d 2^{\text {cre-ERT2 }} N r p 1^{\mathrm{f} / \mathrm{f}}$ as $\left.\mathrm{KO}\right)^{28}$. Although Id 2 has been reported to be expressed in $\mathrm{T}_{\text {reg }}$ cells, dendritic cells (DCs), and neutrophils ${ }^{29-31}$, neither the Nrp1 expression level on these cells nor the number of these cells was affected in $I d 2^{\text {cre-ERT2 }} N r p 1^{\mathrm{f} / \mathrm{f}}$ mice following administration of tamoxifen for 5 days (Extended Data Fig. 2a-f). The efficiency of Nrp1 depletion in ILC2s exceeded 80\% (Extended Data Fig. 2g). Compared with littermate WT controls, KO mice did not show any differences in the ILC2 compartment under steady state, as indicated by percentage and absolute number of ILC2s, production of IL-5 and IL-13, and lung histology (Extended Data Fig. $2 \mathrm{~h}-\mathrm{m})$. As ILC2s play critical roles in controlling worm infection, we next identified whether Nrp1 regulated ILC2 function in the setting of helminth infection. Despite normal function in the steady state, we observed a significant decrease of IL- 5 and IL-13 production by ILC2s in KO mice infected with Nippostrongylus brasiliensis (N. brasiliensis) (Extended Data Fig. 3a-e), suggesting that Nrp1 is essential to maintain ILC2 function under the condition of type 2 inflammation. Nonetheless, the absolute count of lung ILC2s was unaffected in $\mathrm{KO}$ mice, suggesting that Nrp1 is not necessary for ILC2 survival or proliferation (Extended Data Fig. 3f). Consistent with reduced type 2 cytokines, less eosinophil infiltration and higher worm burden were observed in KO mice (Extended Data Fig. $3 g-i$ ).

Both TGF $\beta 1$ and ILC2s have been shown to contribute to the pathogenesis of pulmonary fibrosis by promoting activation of myofibroblasts and the deposition of extracellular matrix ${ }^{32-35}$. In a mouse lung fibrosis model induced by bleomycin, we observed an increase of TGF $\beta 1$ release in bronchoalveolar lavage fluid (BALF) and an elevation of ILC2 cell numbers during the development of disease (Fig. 4a,b). Intriguingly, the expression levels of Nrp1 and ST2 were enhanced on ILC2s concomitantly (Fig. 4c,d). Thus, we hypothesized that Nrp1 might have a critical function in the development of pulmonary fibrosis. During the early phase of bleomycin-induced fibrosis, lung tissue exhibited an acute inflammatory response due to tissue damage (Fig. 4e). We observed reduced neutrophil infiltration in $\mathrm{KO}$ mice compared with the WT controls (Fig. 4e,f). Despite the lack of an effect on the frequency of ILC2s (Fig. 4g), IL-13 production was markedly decreased in KO ILC2s (Fig. 4h), and IL-5 production was only slightly reduced (Fig. 4i). With progression of the disease, we observed a dramatic decrease of lung collagen deposition in $\mathrm{KO}$ mice, as shown by Masson's trichrome staining at 21 days following bleomycin challenge (Fig. 4j). In accordance with the histology data, KO mice showed reduced expression of pulmonary fibrotic genes, including Col1a1, Fn1, and Timp1 (Fig. 4k), and decreased expression of the fibrotic marker alpha-smooth muscle actin ( $\alpha$-SMA) (Fig. 4l). At the cellular level, KO mice mounted defective ILC2 responses, including a marked reduction in IL-13

Fig. 3 | TGF $\beta 1$ induces and maintains Nrp1 expression by lung ILC2s. a-c, mRNA expression level of Tgfb1, Vegfa, and Sema3a in various tissues ( $n=3$ / group). d,e, Sorted ILC2s from LI were cultured for 48 hours in the presence of IL-33 (10 ng/ml), TGF 31 (1 ng/ml), VEGF164 (100 ng/ml), and Sema3a $(100 \mathrm{ng} / \mathrm{ml})$. d, The expression of Nrp1 on ILC2s. e, Percentage of Nrp1+ ILC2s out of total ILC2s ( $n=4 / \mathrm{group})$. $\mathbf{f}, \mathbf{g}$, Sorted ILC2s were stimulated with TGF $\beta 1$ at different concentrations for 48 hours. Frequency of Nrp1+ ILC2s (f) and MFI of Nrp1 on ILC2s (g), detected by flow cytometry $(n=9,8,7,6,6,4$, from the left to the right). $\mathbf{h}$, ILC2s sorted from human peripheral blood were cultured with TGF $\beta 1$ at $1 \mathrm{ng} / \mathrm{ml}$ for 48 hours. MFI of Nrp1 was analyzed ( $n=5 / \mathrm{group}$ ). i, TGF $\beta$ RII expression was analyzed on L and LI ILC2s. $\mathbf{j}$, The concentration of bioactive TGF $\beta 1$ in various tissues was determined by ELISA ( $n=4 / g r o u p$ ). k, Lung mononuclear cells were cultured with anti-TGF $\beta 1$ or isotype control antibodies, and the frequency of Nrp1+ILC2s was determined ( $n=8 /$ group). LPLs from $\mathrm{LI}(\mathbf{I})$ or $\mathrm{SI}(\mathbf{m})$ of CD45.2 mice were cultured with L mononuclear cells from CD45.1 mice for 48 hours in the presence of anti-TGF $\beta 1$ or isotype control antibodies. Frequency of CD 45.2+Nrp1+ILC2s was analyzed ( $n=8$ in $\mathbf{l}, n=6$ in $\mathbf{m}$ ). $\mathbf{n}$, ILC2s sorted from LI were cultured for 48 hours in the presence of TGF $\beta 1$ $(1 \mathrm{ng} / \mathrm{ml})$ with LY2109761 at $5 \mu \mathrm{M}$ or SB431542 at $1 \mu \mathrm{M}$. MFI of Nrp1 was determined by flow cytometry ( $n=4 / \mathrm{group})$. o, ILC2s were stimulated with TGF $\beta 1$ in RPMI 1640 complete medium without fetal bovine serum for 1 hour and the phosphorylation of Smad2 (S465/S467)/Smad3 (S423/S425) was determined by flow cytometry. $\mathbf{p}$, ILC2s sorted from LI were cultured for 48 hours in the presence of TGF $\beta 1(1 \mathrm{ng} / \mathrm{ml})$ with SIS3 HCl at $2 \mu \mathrm{M}$. MFI of Nrp1 was determined by flow cytometry ( $n=4 /$ group). Each symbol represents an individual mouse $(\mathbf{a}-\mathbf{c}, \mathbf{j})$. Data are representative of $2-4$ independent experiments and are presented as mean \pm s.e.m. For statistical analysis, the following tests were used. a-c,e,j,n,p, One-way ANOVA. h,k-m, Two-tailed paired Student's $t$ test. 
and IL-5 production by lung ILC2s (Fig. $4 \mathrm{~m}-\mathrm{p}$ ), despite the lack of change in the number of ILC2s (Fig. 4q). In addition, a statistically significant decrease in the number of $\alpha-\mathrm{SMA}^{+}$myofibroblasts, a key component of fibrosis, was observed in KO mice (Fig. 4r). Together, these data demonstrate that Nrp1 is critical in regulating lung ILC2 function and the development of pulmonary fibrosis.

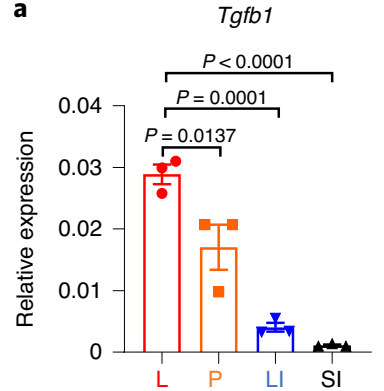

d

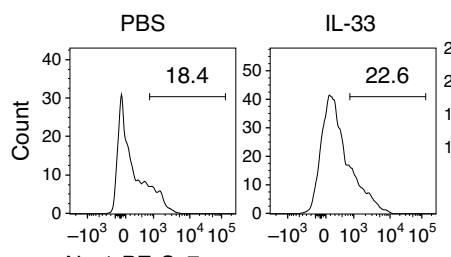
Nrp1-PE-Cy7

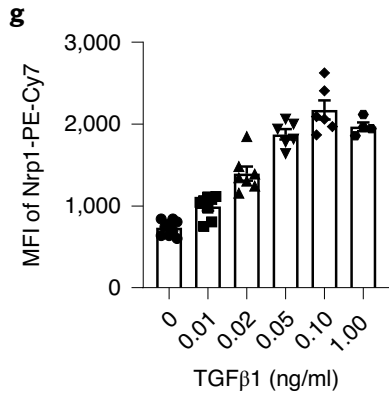

j

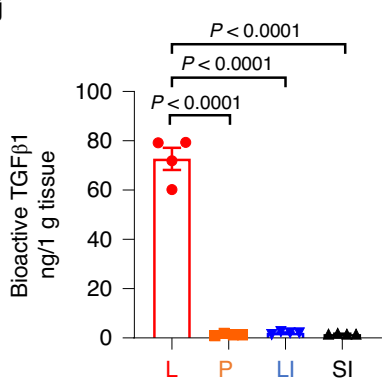

n

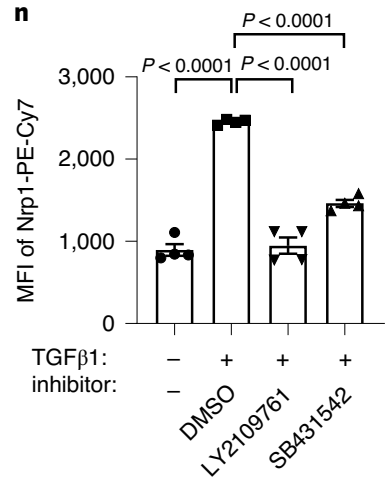

k b

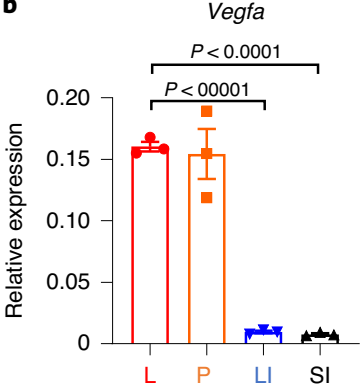

C Sema3a

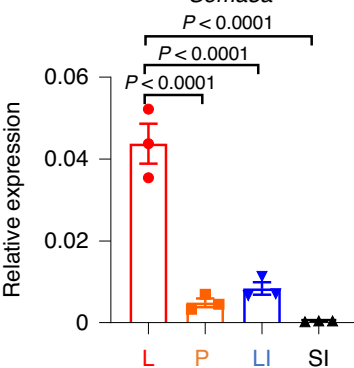

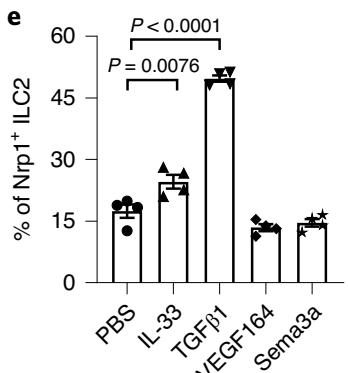

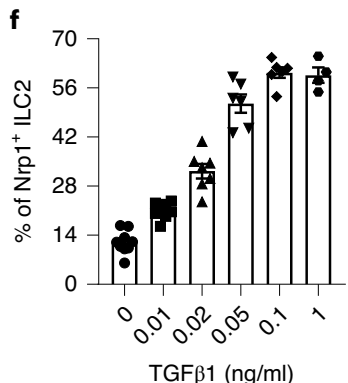

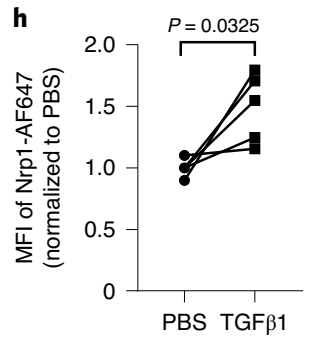

i
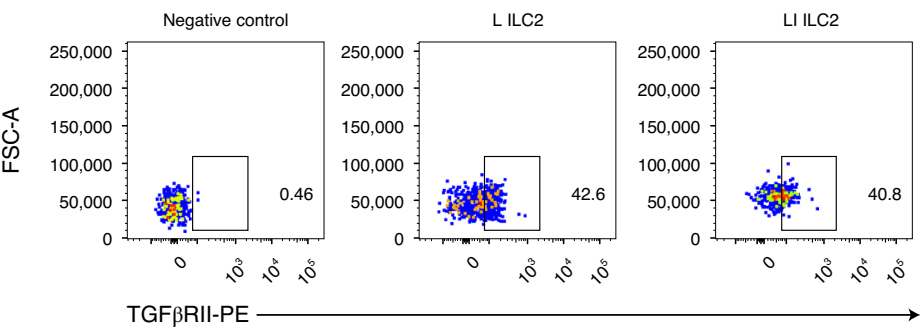

I

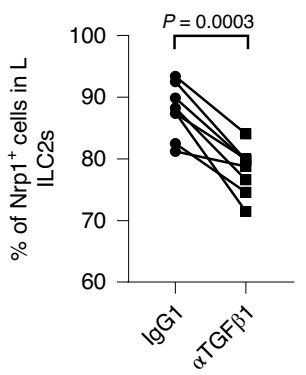

TGF $\beta$ RIII-PE
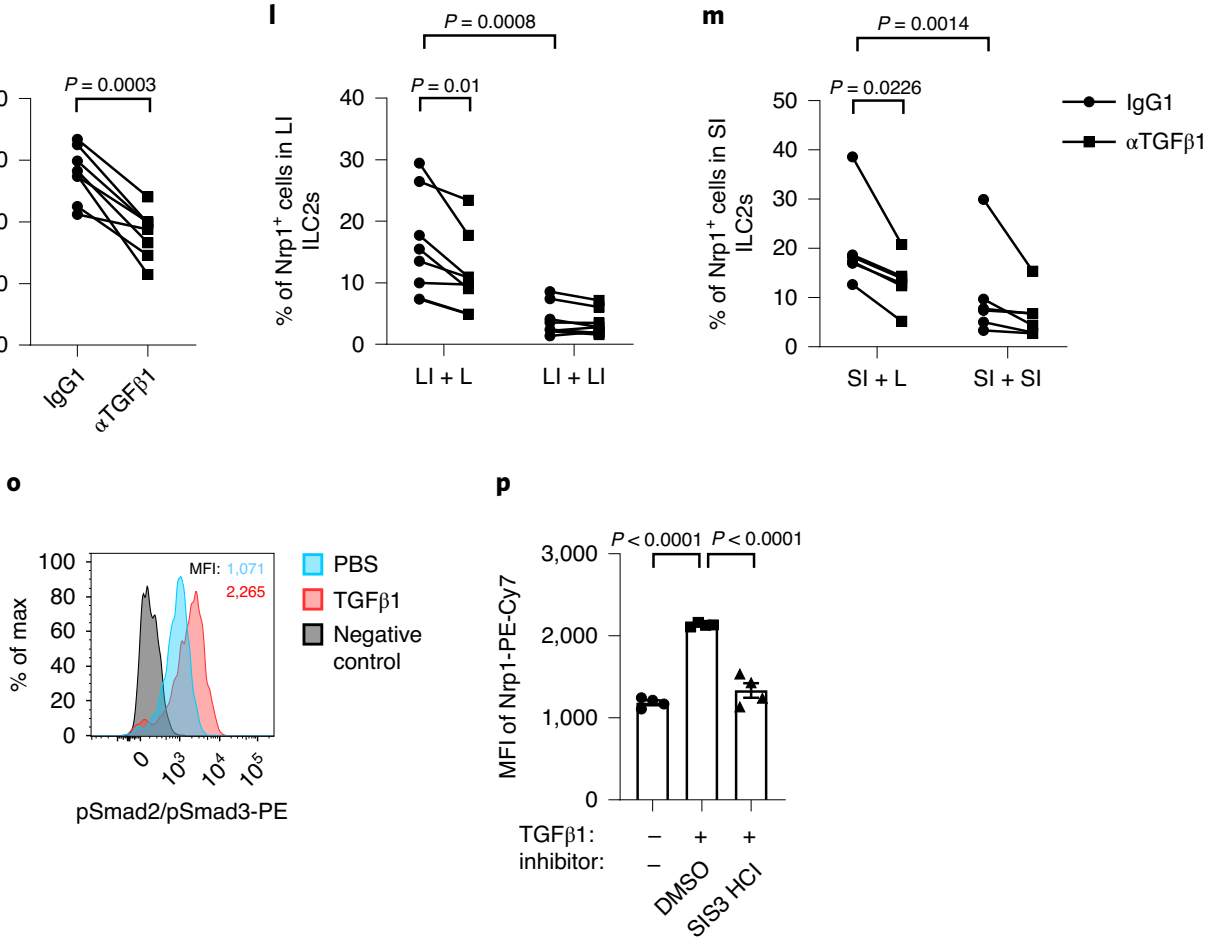


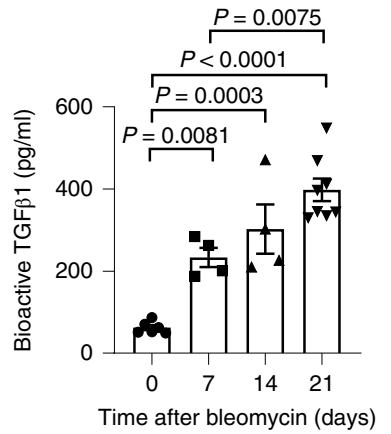

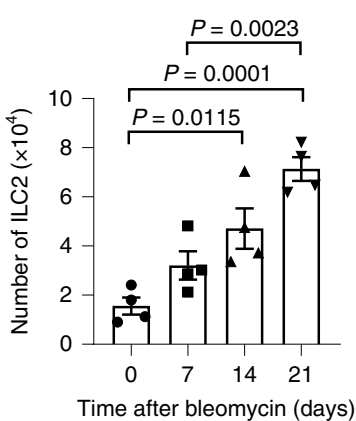

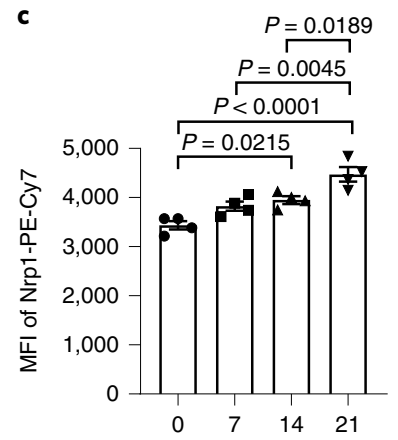

Time after bleomycin (days) d

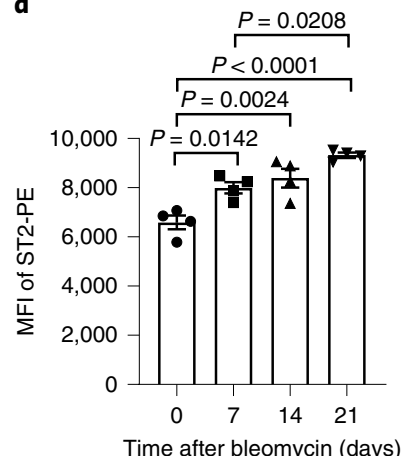

e

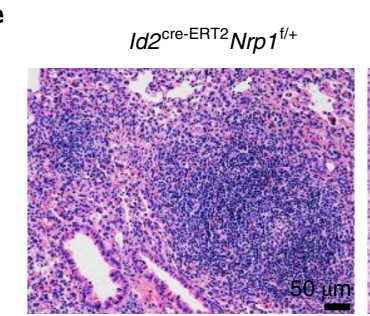

j

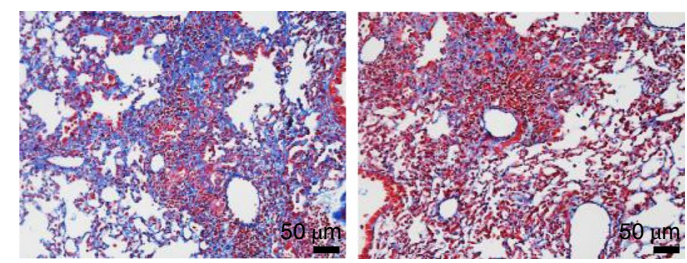

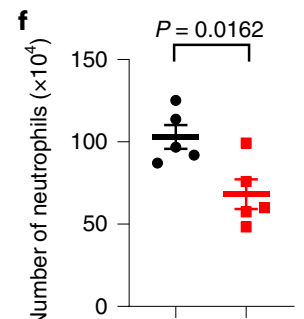

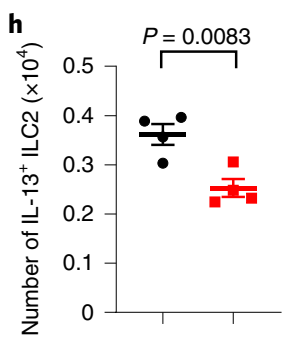

Timp1
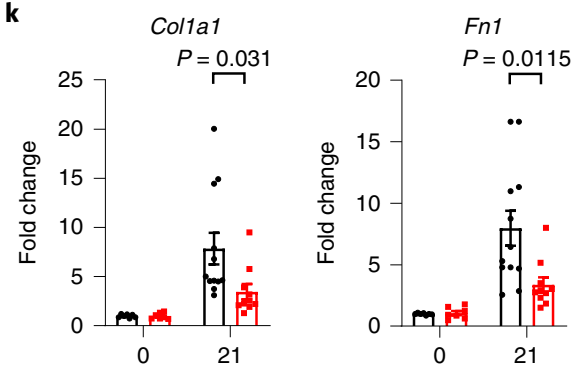

Time after bleomycin (days)
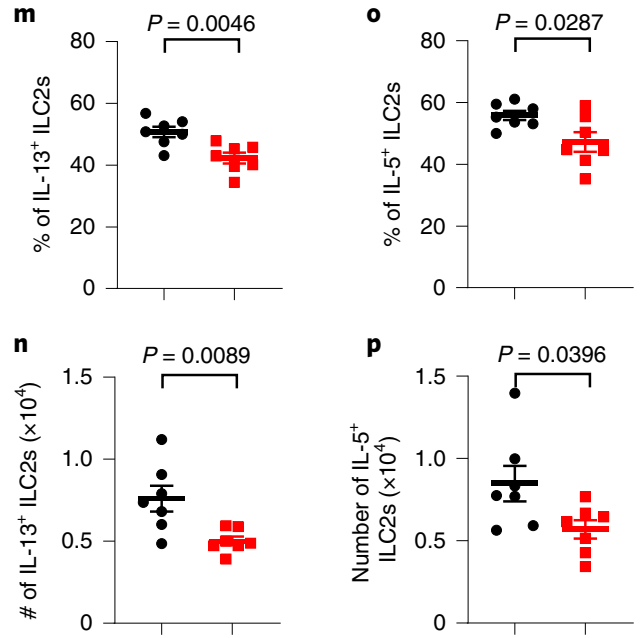

Nrp1 regulates lung ILC2 function in a cell-intrinsic manner. ILC2s are the predominant cells for IL-5 production in the lung, and Il5RFP-cre $(\mathrm{R} 5 /+)$ mice have been used for selective deletion

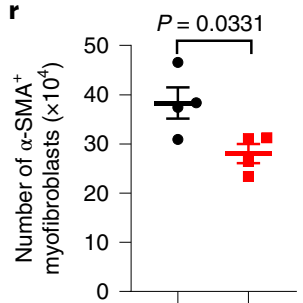

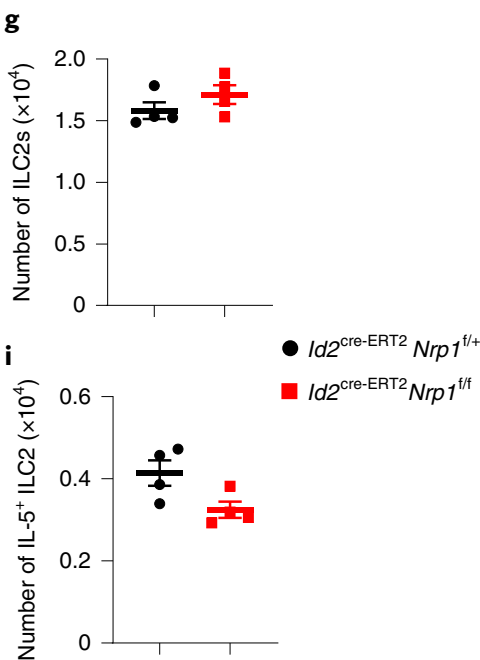
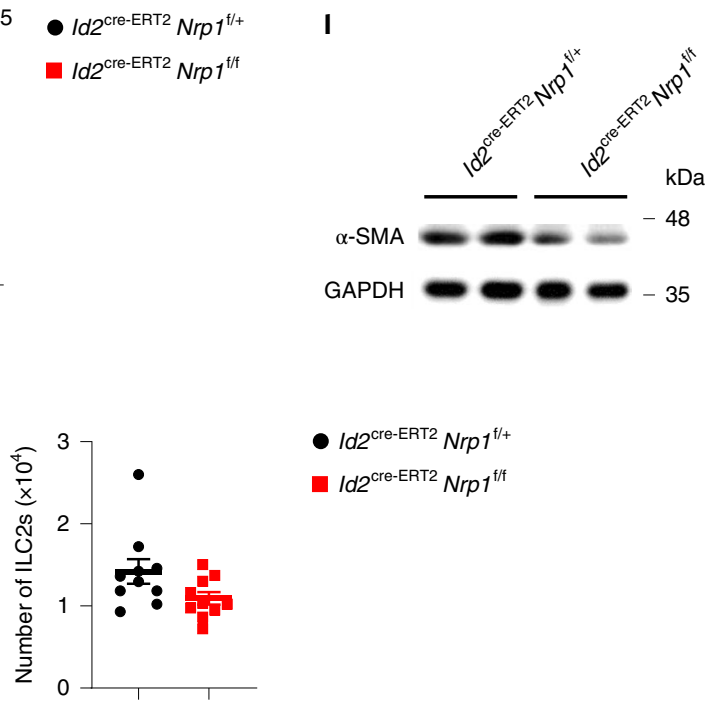

- $\operatorname{ld} 2^{\mathrm{cre}-\mathrm{ERT} 2} \mathrm{Nrp} 1^{\mathrm{f} /+}$

Id $2^{\text {cre-ERT2 }} \mathrm{Nrp1}^{\mathrm{f} / \mathrm{f}}$

of genes in ILC2 $\mathrm{s}^{36}$. Therefore, to validate the cell-intrinsic role of Nrp1 in ILC2s, we generated $\mathrm{R} 5 /+N r p 1^{\mathrm{f} / \mathrm{f}}$ mice. ILC2s from $\mathrm{R} 5 /+N r p 1^{\mathrm{f} / \mathrm{f}}$ mice showed efficient deletion of Nrp1 (Extended Data 
Fig. 4 | Ablation of Nrp1 in ILC2s ameliorates pulmonary fibrosis. a-d, Wild-type mice were treated with bleomycin (BLM) (2 mg/kg body weight) intranasally to induce lung fibrosis. BALF and lungs were collected at the indicated time points. $\mathbf{a}$, The concentration of TGF $\beta 1$ in the BALF was determined by ELISA ( $n=6,4,4,8$ in D0, D7, D14, D21.). b, Absolute count of lung ILC2s ( $n=4 / g r o u p)$. c,d, MFI of Nrp1 and ST2 on lung ILC2s ( $n=4 / g r o u p)$. e-r, $I d 2^{\text {cre-ERT2 }} \mathrm{Nrp}^{t / /+}$ and $/ d 2^{\text {cre-ERT2 }} \mathrm{Nrp}^{7 / /}$ mice were injected peritoneally with tamoxifen ( $2 \mathrm{mg} /$ day) for 5 days to knock down Nrp1 expression in the ILC lineage. After 3 days, mice were treated with $2 \mathrm{mg} / \mathrm{kg}$ bleomycin intranasally. $\mathbf{e}-\mathbf{i}$, Lung tissues were collected and analyzed on day 7. e, H\&E staining of lung tissue. $\mathbf{f}-\mathbf{i}$, Absolute counts of neutrophils, ILC2s, IL-13+ ILC2s, and IL- $5^{+}$ILC2s in L ( $n=5 /$ group in $\mathbf{f}, n=4 /$ group in $\mathbf{g}-\mathbf{i}$ ). $\mathbf{j}-\mathbf{r}$, L tissues were collected on day 21 after bleomycin treatment. $\mathbf{j}$, Masson's trichrome staining of $L$ tissue. $\mathbf{k}$, mRNA level of the indicated fibrotic genes was determined by qRT-PCR $(n=7 / g r o u p$ on D0, $n=12$ (black group), 10 (red group) on D21). I, $\alpha$-SMA expression level by Immunoblot assay. m,n, Frequency and absolute count of IL-13+ lung ILC2s ( $n=7 /$ group). o,p, Frequency and absolute number of IL-5+ lung ILC2s ( $n=7 /$ group). q,r, Absolute counts of lung ILC2s ( $n=10 / g r o u p)$ and $\alpha-S M A^{+}$pulmonary myofibroblasts ( $n=4 /$ group). Each symbol represents an individual mouse (a-d,f-i, $\mathbf{m}-\mathbf{r})$. Data are representative of 3 independent experiments and are presented as mean \pm s.e.m. For statistical analysis, the following tests were used. $\mathbf{a}-\mathbf{d}$, One-way ANOVA. $\mathbf{f}-\mathbf{i}, \mathbf{k}, \mathbf{m}-\mathbf{r}$, Two-tailed unpaired Student's $t$ test.

Fig. 4a). R5/+Nrp1 $1^{\mathrm{f} / \mathrm{f}}$ mice did not show any defects in the lung ILC2 compartment under steady state (Extended Data Fig. 4b-f), whereas production of IL- 5 and IL-13 by ILC2s was reduced when $\mathrm{R} 5 /+N r p 1^{\mathrm{f} / \mathrm{f}}$ mice were infected with $N$. brasiliensis, accompanied by less eosinophil infiltration and higher worm burden in the lung (Extended Data Fig. 5a-i). These data suggest that the cell-intrinsic support of ILC2 function by Nrp1 during inflammation is important for anti-helminth immunity. When R5/+mice were treated with bleomycin to induce lung fibrosis, the percentage of lung ILC2s was increased significantly (Extended Data Fig. 6a). By contrast, the frequency of IL- $5^{+}$non-ILC2 cells remained unchanged (Extended Data Fig. 6a). Upon bleomycin challenge, R5/+Nrp1 $1^{\mathrm{f} / \mathrm{f}}$ mice displayed substantially reduced collagen accumulation in the lung compared with that in control mice (Fig. 5a). Consistently, Nrp1 deficiency resulted in substantially decreased expression of fibrosis-associated genes, including Col1a1, Fn1, and Timp1 (Fig. $5 b)$. The protein level of $\alpha$-SMA in the lung of Nrp1-deficient mice was also reduced (Fig. 5c). At the cellular level, we observed a reduction in the frequencies and total counts of IL-5- or IL-13-producing lung ILC2s in R5/+Nrp $1^{\mathrm{f} / \mathrm{f}}$ mice compared with in control mice (Fig. $5 \mathrm{~d}-\mathrm{g})$. The number of $\alpha-\mathrm{SMA}^{+}$myofibroblasts was also reduced in $\mathrm{R} 5 /+N r p 1^{\mathrm{f} / \mathrm{f}}$ mice (Fig. $5 \mathrm{~h}$ ).

To further corroborate the cell-intrinsic effect of Nrp1 on ILC2s, ILC2s isolated from R5/+ mice (WT) or R5/+Nrp ${ }^{\mathrm{f} / \mathrm{f}}$ mice (KO) were transferred to Rag2 $2^{-/-} I l 2 \mathrm{rg}^{-/-}$mice, which were subsequently challenged with bleomycin to induce fibrosis. Both WT and KO ILC2s reconstituted successfully in the lungs of recipient mice, and the total number of ILC2s was comparable between the two groups of recipients (Fig. 5i and Extended Data Fig. 6b,c). Strikingly, mice that received KO ILC2s developed greatly diminished pulmonary fibrotic responses, as indicated by reduced collagen deposition, decreased fibrotic gene expression, and reduced $\alpha-\mathrm{SMA}^{+}$myofibroblast activation (Fig. 5j-1). Consistently, a significant decrease of IL-13 and IL-5 production was observed in KO ILC2s (Fig. 5m,n and Extended Data Fig. 6d,e). Taken together, the results indicate that Nrp1 acts in a cell-autonomous manner in regulating the function of lung ILC2s in the progress of fibrosis.
Next, we analyzed the expression of NRP1 in human ILC2s isolated from peripheral blood, BALF, and lung tissue from people with lung cancer who had fibrosis. Lung tissues were collected from fibrotic lesions. Importantly, we found that NRP1 was highly expressed on ILC2s from BALF and lung tissue, but not on ILC2s from peripheral blood (Fig. 5o,p). In addition, compared with those in people without fibrosis, BALF ILC2s in people with fibrosis had higher levels of NRP1 expression, suggesting that NRP1 may be involved in the progression of human pulmonary fibrosis (Fig. 5q).

The TGFß1-Nrp1 axis enhances ILC2 function via ST2 upregulation. To gain insights into the molecular mechanisms responsible for Nrp1 regulation of lung ILC2s function, we performed RNA-seq analysis to compare the global transcriptome between Nrp1-deficient and WT lung ILC2s isolated from bleomycin-treated mice. Gene expression analysis identified 1,034 significantly changed genes (fold change $>1.2, P<0.05$ ) out of 10,928 expressed protein-coding genes in Nrp1-deficient ILC2s. Among these genes, 305 were upregulated and 729 were downregulated (Fig. 6a). Gene Ontology (GO) analysis revealed that genes that were decreased in Nrp1-deficient lung ILC2s showed enrichment in pathways involved in intracellular signal transduction, positive regulation of T cell activation, and cellular response to IL-4 (Fig. 6b). Gene Set Enrichment Analysis (GSEA) showed enrichment in pathways involved in the innate-immune-response-activating signaling pathway, IL-13 production, and regulation of wound healing, which were all associated with the pathogenesis of fibrosis (Fig. $6 \mathrm{c}-\mathrm{e})^{37-39}$. Together, these data suggest that Nrp1 targets multiple signaling pathways and programs to regulate ILC2 function. Consistently, we found the genes that are associated with ILC2 activation, function, and transcriptional control were broadly regulated by Nrp1 (Fig. 6f). To further assess the effect of Nrp1 on the expression of ILC2-associated genes, we conducted qRT-PCR in WT and KO ILC2s. Remarkably, Il1rl1 (encoding ST2, IL-33 receptor), Il4, Il5, and Il9r expression were decreased significantly in Nrp1-deficient ILC2s (Fig. 6g). Although the protein level of IL-13 was reduced in Nrp1-deficient ILC2s, the expression of Il13 genes remained statistically unchanged (Fig. $5 \mathrm{~d}, \mathrm{e}, \mathrm{m}, \mathrm{n}$

Fig. 5 | Nrp1 regulates ILC2s function in a cell-intrinsic manner during fibrosis. a-h, R5/+ and R5/+Nrp $7^{\text {t/f }}$ mice were challenged with bleomycin to induce fibrosis. Lung tissues were collected and analyzed on day 21 of the disease. $\mathbf{a}$, Masson's trichrome staining. $\mathbf{b}$, mRNA levels of the fibrotic genes ( $n=4 /$ group on D0, $n=13$ (black group), 8 (red group) on D21). c, $\alpha$-SMA expression level in L by immunoblot assay. d,e, Frequency and absolute count of IL$13^{+}$ILC2s ( $n=5 /$ group). $\mathbf{f}, \mathbf{g}$, Frequency and absolute count of IL-5+ ILC2s ( $n=5 /$ group). h, Total number of $\alpha-S M A+$ myofibroblast $(n=5 / g r o u p) . \mathbf{i}-\mathbf{n}$, We

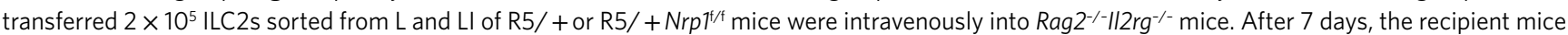
were given bleomycin intranasally. Lung tissues were collected and analyzed on day 21 of fibrosis. $\mathbf{i}$, The reconstitution efficiency of ILC2s in L. Cells were gated on CD45+lin- live cells. j, Masson's trichrome staining. $\mathbf{k}$, mRNA levels of the fibrotic genes ( $n=4 /$ group). I, Total number of $\alpha$-SMA ${ }^{+}$myofibroblasts ( $n=4$ /group). $\mathbf{m}, \mathbf{n}$, Frequency and absolute count of IL-13+ ILC2s ( $n=4 /$ group). (o) ILC2s in human peripheral blood, BALF, and lung tissue were analyzed

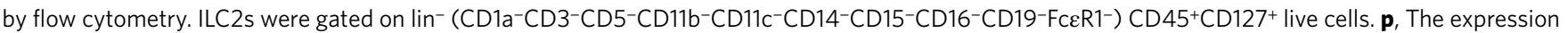
level of NRP1 on human ILC2s from various tissues. q, Frequency of NRP1+ILC2s in BALF from people with lung cancer with or without fibrosis ( $n=5$ (non-fibrosis), $n=3$ (fibrosis)). Each symbol represents an individual mouse (d-h,k-n). Data are representative of two or three independent experiments and are presented as mean \pm s.e.m. For statistical analysis, a two-tailed unpaired Student's $t$ test was used. 
and Fig. 6f,g). Among the significantly changed genes, Il1rl1 (ST2) downregulation was also confirmed at the protein level by flow cytometry (Fig. 6h).

a
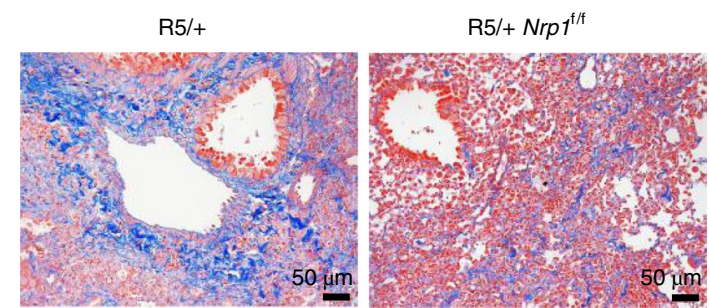

c

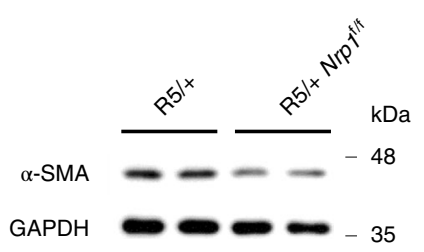

As the IL-33 receptor, ST2 is the key regulator that determines the magnitude of ILC2 activation and the level of type 2 cytokines. Hence the downregulation of ST2 presumably accounts for reduced type 2

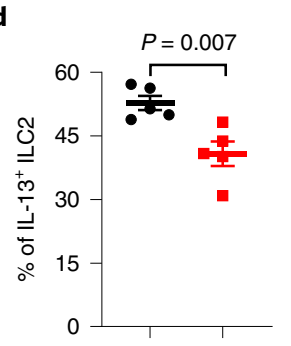

i

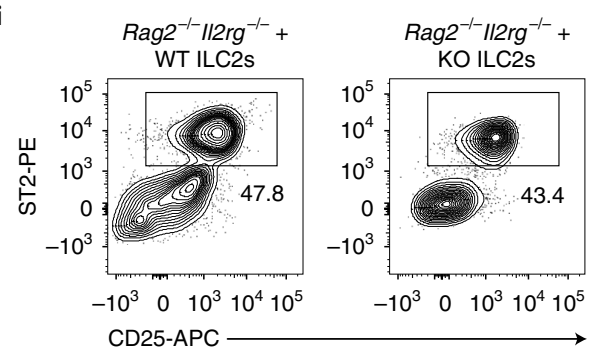

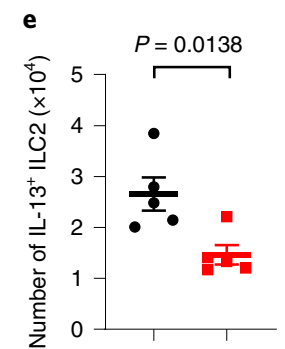

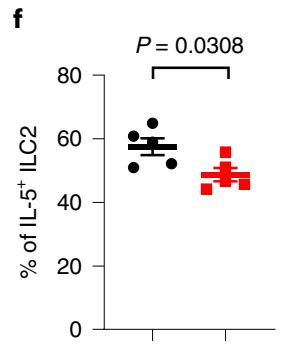

b

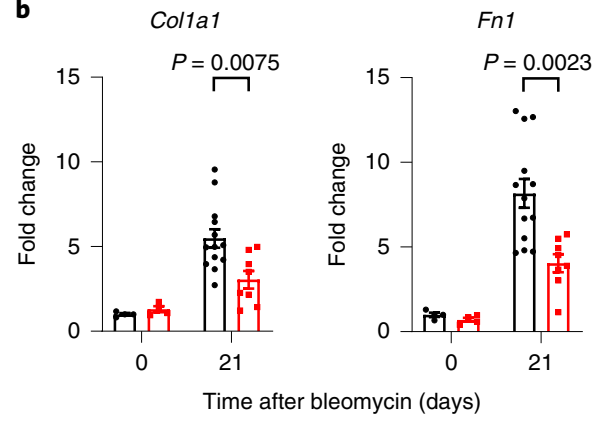

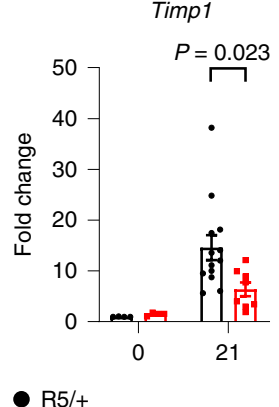

$\mathrm{R} 5 /+\mathrm{Nrp} 1^{\mathrm{f} / \mathrm{f}}$
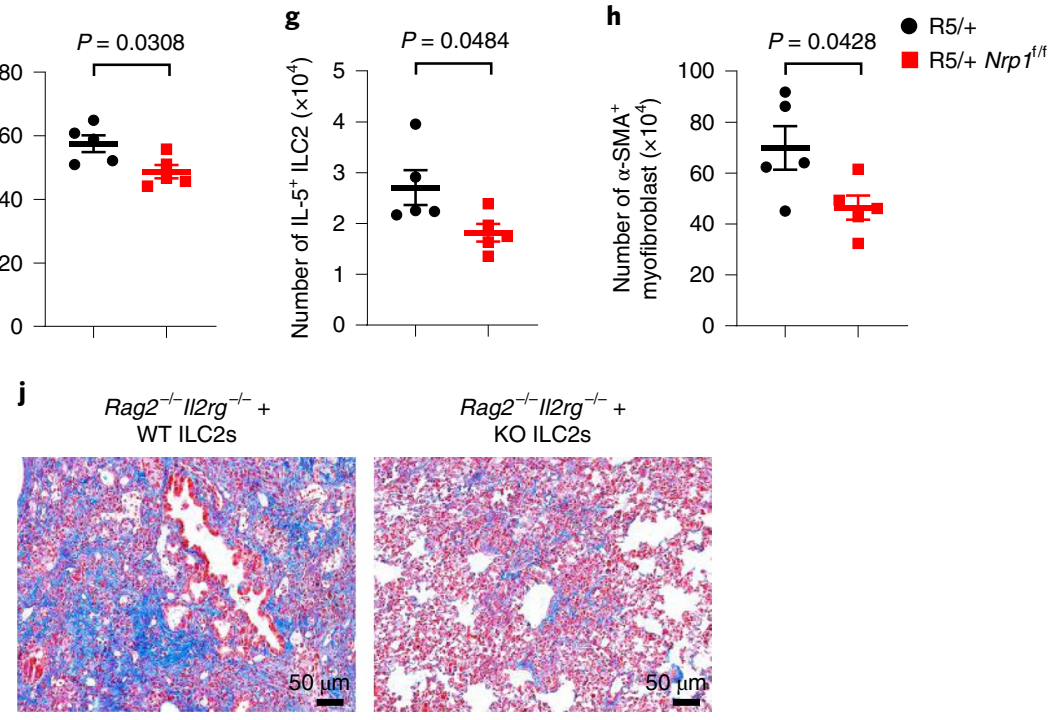

- $\operatorname{Rag}^{-1-} \| 2 \mathrm{rg}^{-1-}+$ WT ILC2s

- Rag2 $^{-1-} I I 2 \mathrm{rg}^{-/-}+\mathrm{KO}$ ILC2s
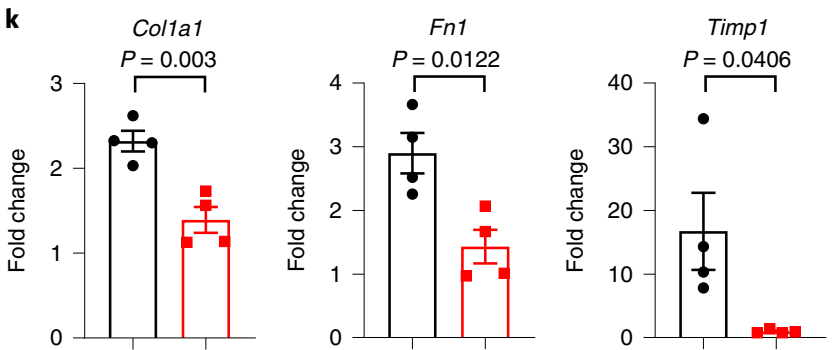

I

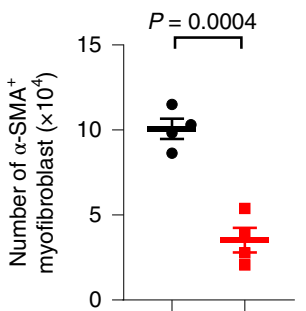

m
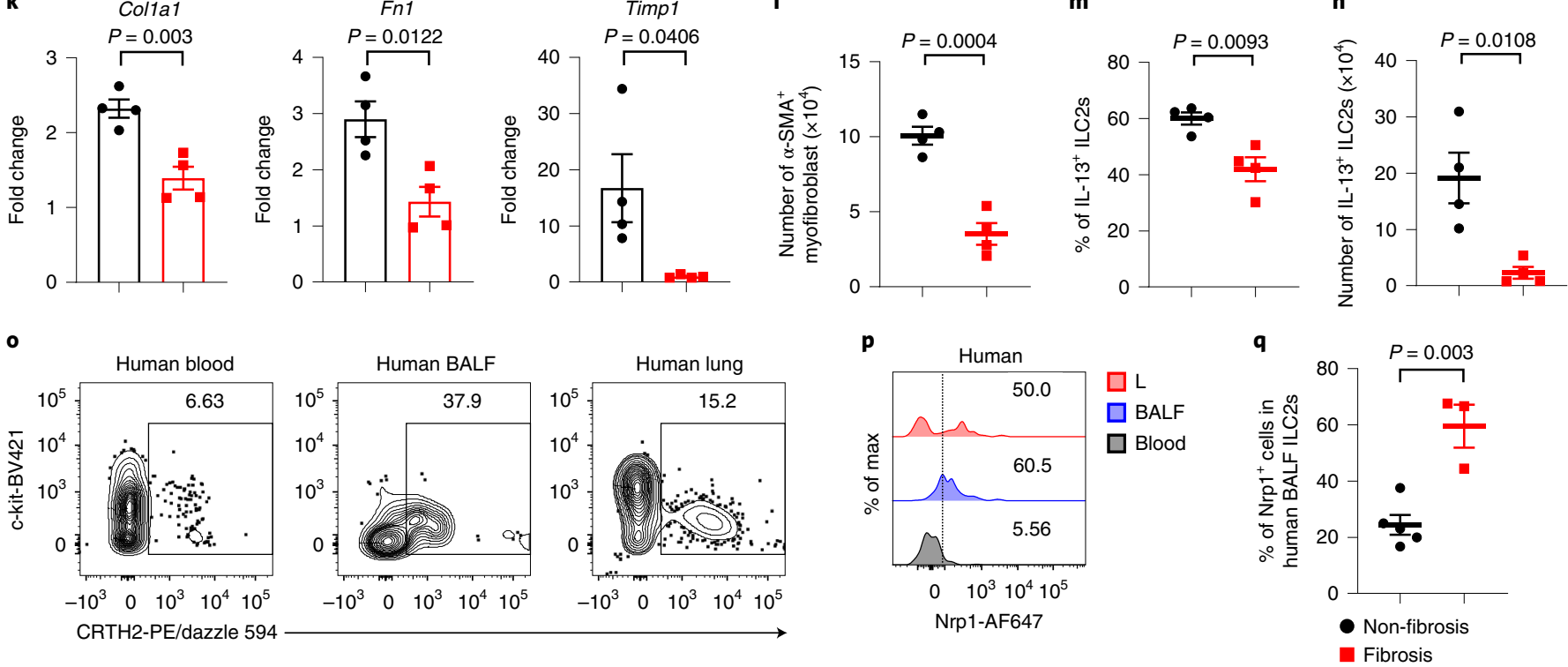
a

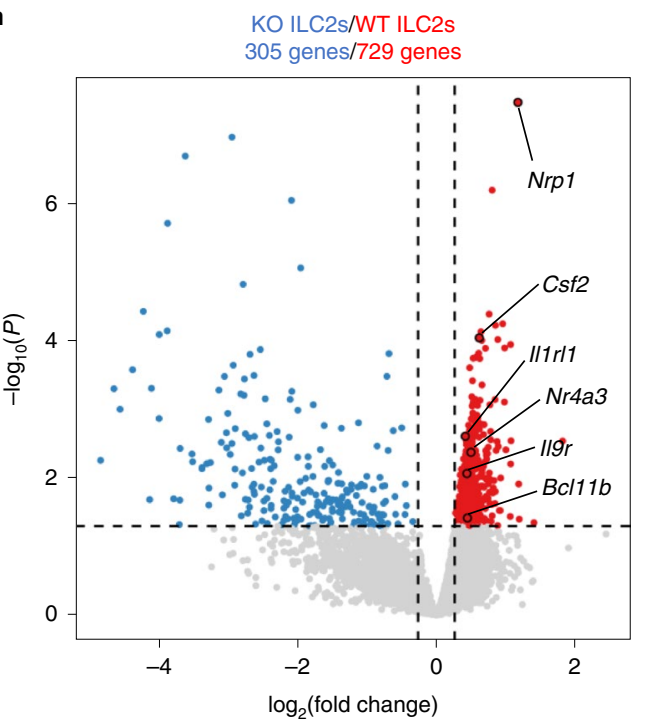

b

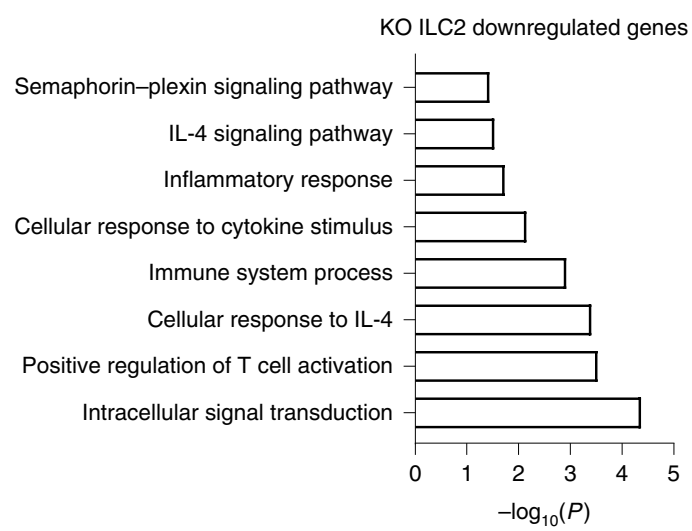

c

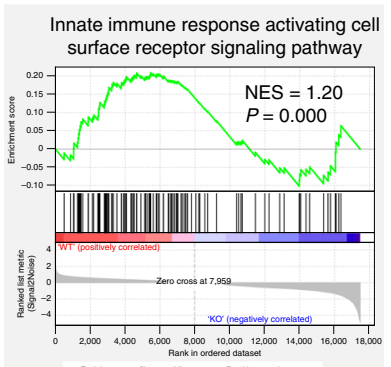

f

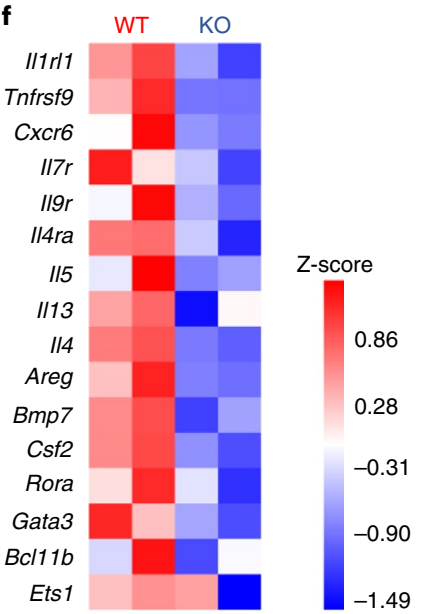

d

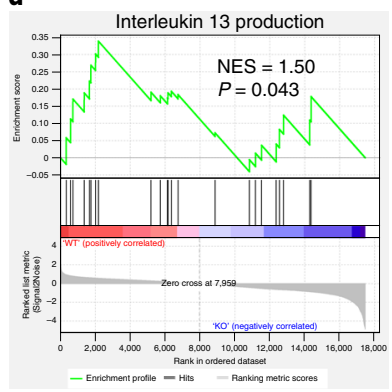

g

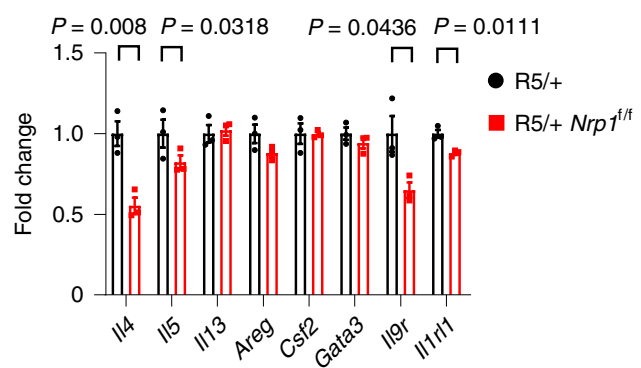

e

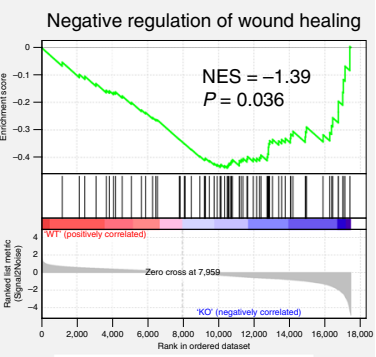

h

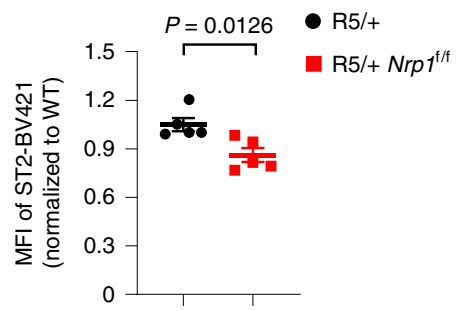

i

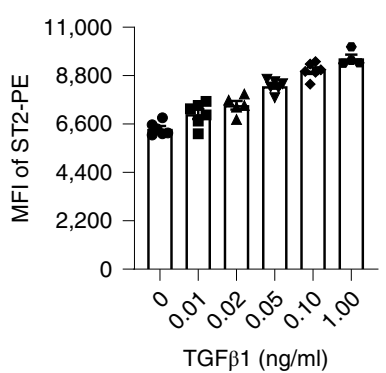

j

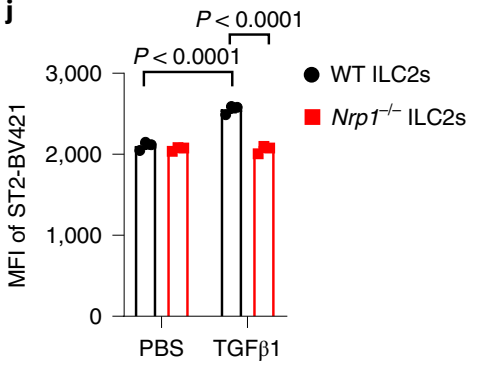

$\mathbf{k}$

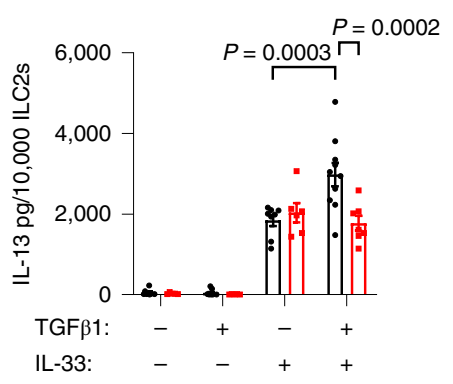

cytokines production in Nrp1-deficient ILC2s. To further determine the effect of Nrp1 on the regulation of ST2 in vitro, purified ILC2s were treated with TGF $\beta 1$ for 48 hours. We found that ST2 expression was upregulated upon TGF $\beta 1$ treatment in a dose-dependent manner (Fig. 6i). We next asked whether ST2 upregulation induced by TGF $\beta 1$ was dependent on Nrp1. Remarkably, we found the enhanced 
Fig. 6 | Nrp1 promotes ILC2 function through upregulation of ST2. a-f, RNA-seq of sorted ILC2s from bleomycin-treated R5/+ (WT) and R5/+Nrp7//f $(K O)$ mice. $\mathbf{a}$, Volcano plot of differentially expressed genes ( $\log _{2}$ (fold change) $>1.2 ; P<0.05$ ) in ILC2s between WT and KO mice. Upregulated genes and downregulated genes are highlighted in blue and red. b, GO analysis of KO ILC2 downregulated genes was performed using DAVID. c-e, GSEA was performed using the webtool from Broad Institute. NES, normalized enrichment score. f, Heatmap of ILC2-characteristic genes in WT and KO ILC2s. $\mathbf{g}$, mRNA expression of ILC2 function-related genes in WT and KO ILC2s ( $n=3 /$ group). $\mathbf{h}$, MFI of ST2 on lung ILC2s from bleomycin-treated R5/+ and $\mathrm{R} 5 /+\mathrm{Nrp}^{\mathrm{t} / \mathrm{f}}$ mice was detected by flow cytometry ( $n=5 /$ group). i, Sorted lung ILC2s were stimulated by TGF $\beta 1$ at different concentrations for 48 hours and the expression of ST2 was determined by flow cytometry $(n=6,6,5,6,6,4$ from left to right). j, Sorted lung ILC2s were cultured with TGF $\beta 1$ at $1 \mathrm{ng}$ / $\mathrm{ml}$ for 24 hours, and the MFI of ST2 on lung ILC2s was detected by flow cytometry ( $n=3 /$ group). k, Sorted ILC2s were primed with TGF $\beta 1$ at $1 \mathrm{ng} / \mathrm{ml}$ for 24 hours, and then were stimulated with IL-33 at $10 \mathrm{ng} / \mathrm{ml}$ for another 24 hours. IL- 13 in the supernatants was detected by ELISA ( $n=9,5,9,5,8,6,10,7$ from left to right). Each symbol represents an individual mouse (h). Data are representative of at least three independent experiments and are presented as mean \pm s.e.m. For statistical analysis, the following tests were used. $\mathbf{g}, \mathbf{h}$, Two-tailed unpaired Student's $t$ test. $\mathbf{j}, \mathbf{k}$, One-way ANOVA.

ST2 expression induced by TGF $\beta 1$ was abolished in Nrp1-deficient ILC2s (Fig. 6j). To address whether Nrp1-mediated ST2 upregulation is responsible for enhanced ILC2 activation, ILC2s were treated with TGF $\beta 1$ first, and subsequently stimulated with IL-33. Interestingly, ILC2s pretreated with TGF $\beta 1$ were more sensitive to IL-33-induced IL-13 upregulation than were those without pretreatment, presumably owing to the increased ST2 expression induced by TGF $\beta 1$ (Fig. $6 \mathrm{k})$. However, this TGF $\beta 1$-sensitized IL-13 upregulation by IL-33 was abolished in Nrp1-deificeint ILC2s (Fig. 6k). Therefore, our results support the view that the TGF $\beta 1-\mathrm{Nrp} 1$ axis enhances ILC2 function via upregulation of ST2.

Blockade of Nrp1 alleviates pulmonary fibrosis. Given that Nrp1 boosts lung-resident ILC2s activation and promotes pulmonary fibrosis, Nrp1 may be a potential therapeutic target for lung fibrosis. To determine whether blockade of Nrpl could ameliorate the development of pulmonary fibrosis, we administered EG00229, a selective NRP1 antagonist that binds to the b1 domain of Nrp1 (ref. ${ }^{40}$ ), to bleomycin-induced mice intraperitoneally (Fig. 7a). EG00229 administration markedly improved the survival rate compared with control DMSO treatment (Fig. 7b). Moreover, mice treated with EG00229 had reduced airway inflammation and less severe lung fibrosis than did the control group, as revealed by H\&E staining and Masson's trichrome staining (Fig. 7c and Extended Data Fig. 7a). The expression of fibrosis-associated genes and the fibrotic marker $\alpha$-SMA protein were also decreased in the treatment group (Fig. 7d,e). To identify the cells targeted by EG00229, we analyzed Nrp1 expression in lung leukocytes. We found that Nrp1 was highly expressed in lung ILC2s, macrophages, DCs, and $\mathrm{T}_{\text {reg }}$ cells, which was consistent with previous reports (Extended Data Fig. $7 \mathrm{~b})$. Although the frequency of these $\mathrm{Nrp}^{+}$cells was unaffected (Extended Data Fig. 7c-e), we observed a significant reduction in the frequency and total counts of IL-13- and IL-5-producing ILC2s in mice treated with EG00229, which was consistent with the changes in R5/+Nrp1 $1^{\mathrm{f} / \mathrm{f}}$ mice (Fig. $7 \mathrm{f}-\mathrm{i}$ ). To evaluate the efficacy of site-specific treatment and avoid unwanted effects of EG00229 on Nrp1-expressing cells outside the lung, EG00229 was administered intranasally to bleomycin-induced mice. Similar to the findings in mice treated with systemically administered EG00229, mice treated with EG00229 through the intranasal route had markedly ameliorated pulmonary fibrosis, as indicated by improved survival, reduced fibrotic responses, and decreased type 2 cytokine production by ILC2s (Extended Data Fig. 8a-i).

To further determine whether the therapeutic effect of EG00229 was dependent on ILC2s, we depleted total ILCs with anti-Thy1.2 antibody in bleomycin-induced $R a g 1^{-/-}$mice. Both lung ILCs and Thy $1.2^{+}$ILC2s were depleted efficiently (Fig. 7j). As expected, mice treated with anti-Thy1.2 antibody developed less severe fibrosis than did the control group of isotype-treated mice, as indicated by the reduced expression of fibrotic genes (Fig. 7k), which might be due to the loss of ILC2 cells. Similar to our findings in WT mice, treatment with EG00229 improved the disease compared with DMSO treatment in Rag1 ${ }^{-/-}$mice, as shown by Masson's trichrome staining (Extended Data Fig. 9a). However, the therapeutic effect of EG00229 disappeared in Rag1 $1^{-/-}$mice treated with anti-Thy1.2 antibody (Fig. 7k and Extended Data Fig. 9a), suggesting that the efficacy of EG00229 is dependent on ILC2s. Additionally, in vitro TGF $\beta 1$ pretreatment of human lung mononuclear cells significantly potentiated IL-33-induced production of IL-13 and IL- 5 by lung ILC2s (Fig. 71-n), which was abolished by EG00229. However, EG00229 had no effect on IL-33-induced IL-13 or IL-5 production by ILC2s without TGF $\beta 1$ treatment (Fig. 71-n). These data indicate that Nrp1 is essential for functional activation of human ILC2s by IL-33 in conjunction with TGF $\beta 1$. In combination, these findings point to Nrp1 as a promising target for treating pulmonary fibrosis.

\section{Discussion}

ILC2s play critical roles in regulating tissue homeostasis and inflammation in health and disease. But how they adapt to their local tissue milieu to regulate tissue-specific immunity remains poorly

Fig. 7 | Nrp1 inhibitor EG00229 alleviates pulmonary fibrosis. a-i, Bleomycin-challenged mice were treated with EG00229 (10 mg/kg body weight) or DMSO control intraperitoneally for 3 weeks. $\mathbf{a}$, Schematic of the experimental design. BLM, bleomycin. $\mathbf{b}$, Mice were challenged with bleomycin (15 mg/ $\mathrm{kg}$ ). The survival rate of mice treated with EG00229 or DMSO control was observed for a period of 28 days ( $n=11$ for DMSO, $n=14$ for EG00229). c. Masson's trichrome staining of $L$ tissues. $\mathbf{d}$, mRNA expression of the fibrotic genes in $L(n=6,11,9,6,14,13,6,14,13$ from left to right). e, $\alpha$-SMA expression level in L by Immunoblot assay. $\mathbf{f}, \mathbf{g}$, Frequency and absolute count of IL-13+ ILC2s ( $n=6$ for DMSO, $n=7$ for EG00229). $\mathbf{h , i}$, Frequency and absolute count of IL-5+ ILC2s ( $n=6$ for DMSO, $n=7$ for EG00229). j,k, Bleomycin-challenged Rag1 ${ }^{-1-}$ mice were treated with EG00229 (10 mg/kg body weight) or DMSO control intraperitoneally for 3 weeks. Each group of mice were also received $200 \mu \mathrm{g}$ of anti-Thy 1.2 antibody or isotype control antibody every 3 days throughout the treatment. $\mathbf{j}$, The depletion efficiency of total ILC $\left(C D 45^{+}\right.$lin ${ }^{-}$CD $\left.127^{+}\right)$and ILC2 $\left(C D 45^{+}\right.$lin ${ }^{-}$CD $127^{+}$CD $90^{+}$ST2 $2^{+}$CD $\left.25^{+}\right)$in L was determined by flow cytometry. The top row was gated on CD45+ live lymphocytes, and the bottom row was gated on CD45+CD127+lin-CD90+ live lymphocytes. $\mathbf{k}$, The expression of fibrotic genes was measured by qRT-PCR in each group of Rag1-/- mice $(n=4,6,4,5,4,6,4,5,4,6,4,5$ from left to right). I-n Human lung mononuclear cells were treated with EG00229 at $5 \mu \mathrm{M}$ under various stimulation condition for 48 hours: IL-33, IL-33+EG00229, TGF $\beta 1+$ IL-33, TGF $\beta 1+$ IL-33 + EG00229. I, IL-13 and IL-5 production by human lung ILC2s were determined by flow cytometry. Cells were stimulated with PMA and ionomycin and gated on total ILC2s. $\mathbf{m}, \mathbf{n}$, Frequency of IL-13+ ILC2s and IL-5+ ILC2s ( $n=5 /$ group). Each symbol represents an individual mouse $(\mathbf{d}, \mathbf{f}-\mathbf{i})$. Data are representative of at least three independent experiments and are presented as mean \pm s.e.m. For statistical analysis, the following tests were used. $\mathbf{b}$, ${ }^{\star} P \leq 0.05$ by log-rank test. $\mathbf{d}, \mathbf{f}-\mathbf{i}$, two-tailed unpaired Student's $t$ test. $\mathbf{k}$, One-way ANOVA. m,n, Two-tailed paired Student's $t$ test. 
understood. Here, we have shown that Nrp1 represents a tissuespecific signature for lung ILC2s, which is acquired postnatally by adaption to the lung microenvironment cue, TGF $\beta 1$. Nrp1 is a potent modulator of type 2 immune responses in lung via regulating ILC2 activation and therefore represents a promising therapeutic target for ILC2-associated pathology. a

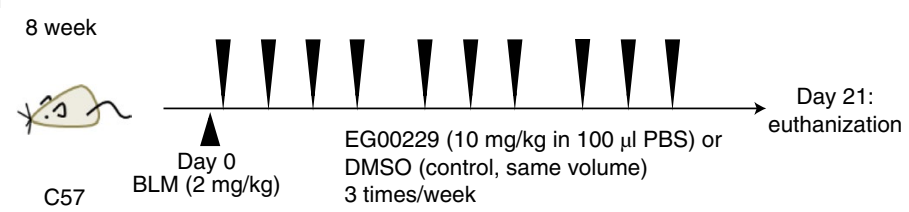

c

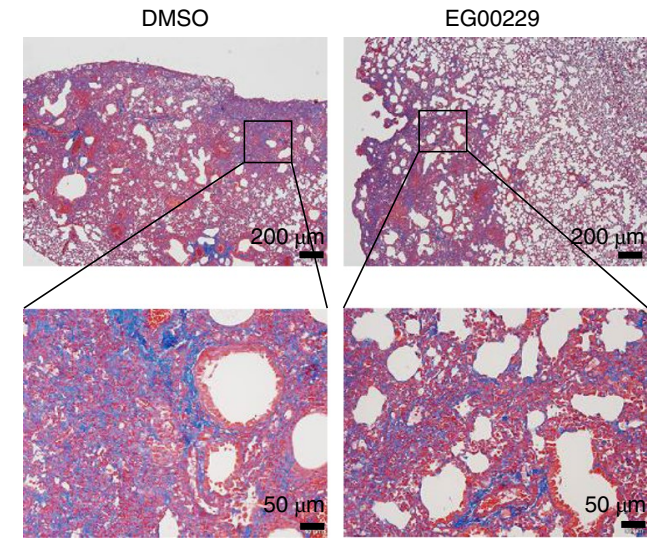

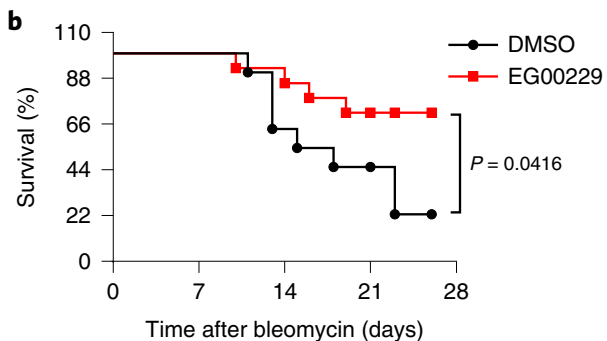

d

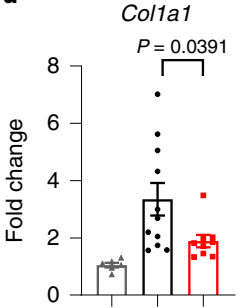

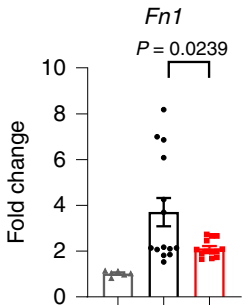
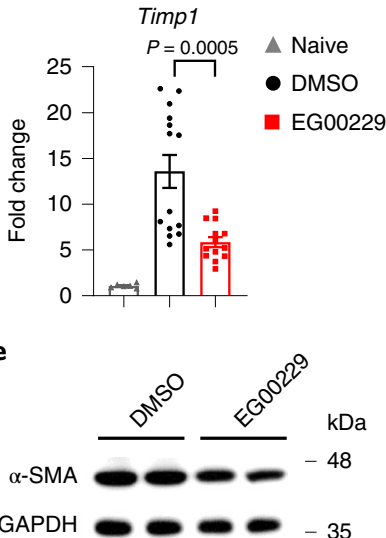

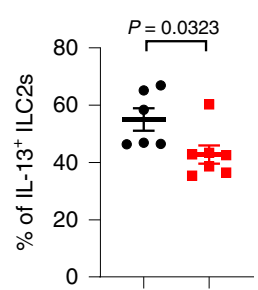

g
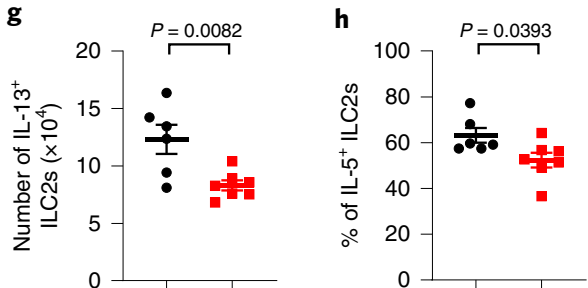

j
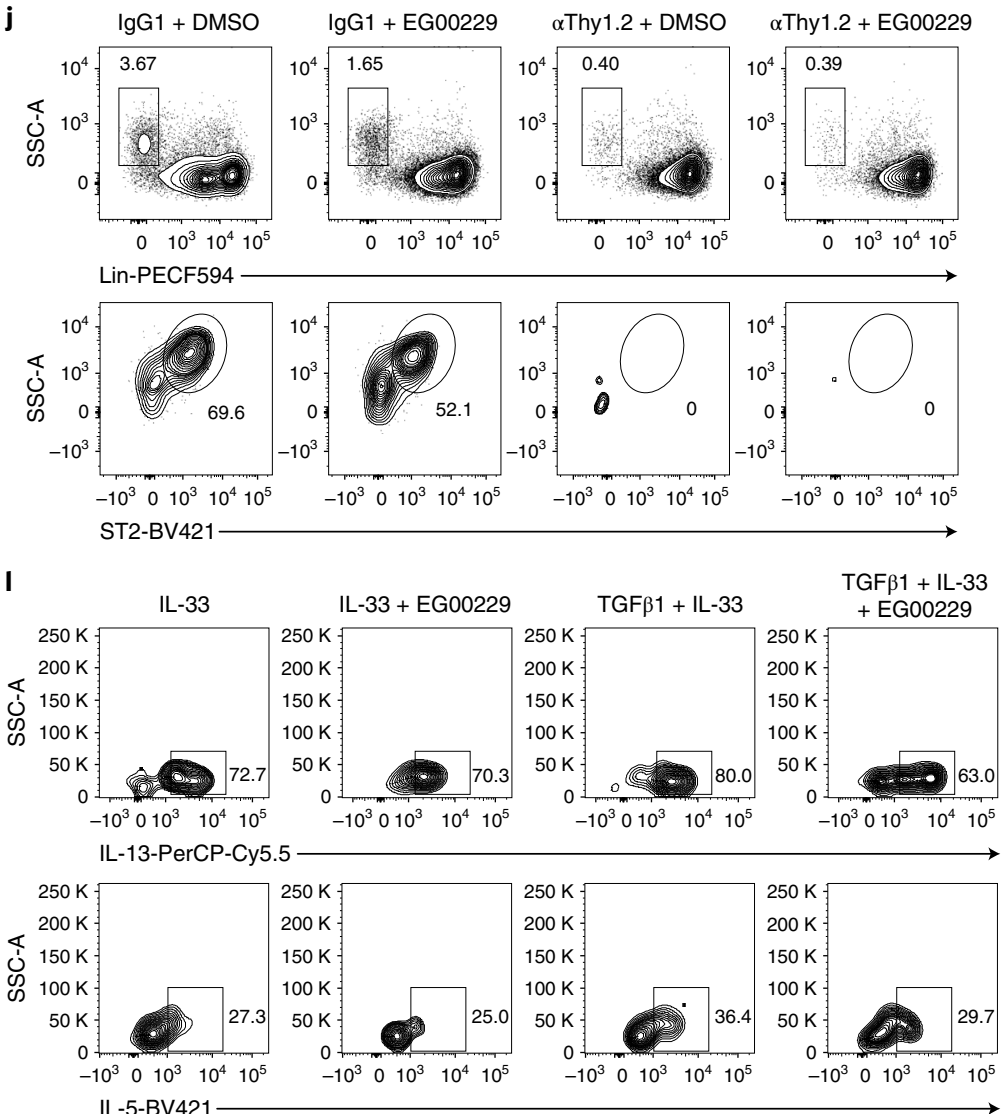

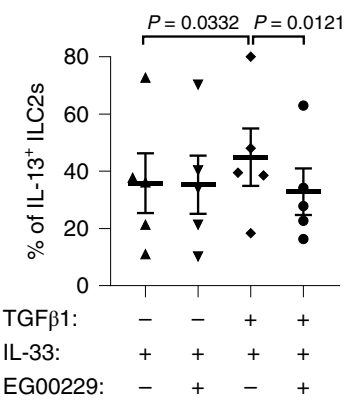

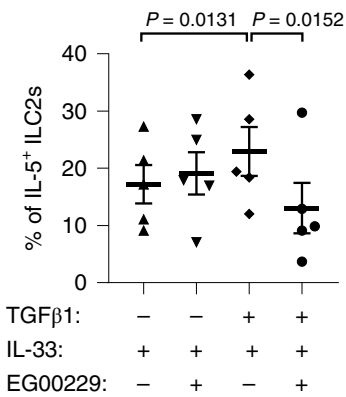


ILC2s establish their tissue residency from the seeding of ILC2 precursors into the tissues, where they acquire tissue-specific transcriptional programs that enable their expression of distinct receptors and development into mature tissue ILC2s. To determine when lung ILC2s acquire the Nrp1 signature during the developmental process, we performed a time-course experiment in mice at ages days $1,7,21$, and 42. Intriguingly, ILC2s from newborn mice did not express Nrp1. By day 7 after birth, Nrp1 expression was observed on around $40 \%$ of ILC2s. The expression level of Nrp1 kept rising and reached $80 \%$ of ILC2s by 6 weeks. These results support the hypothesis that environmental cues are responsible for promoting tissue-specific Nrp1 expression in lung ILC2s. We further found that Nrp1-negative ILC2s acquired Nrp1 expression once they arrived in the lung, whereas Nrp1-positive ILC2s reduced their expression after leaving the lung. These data further corroborate the hypothesis that Nrp1 expression on ILC2s is dependent on lung-derived signals.

TGF $\beta 1$ was identified as the key signal driving Nrp1 expression during the development of lung-resident ILC2s. TGF $\beta 1$ is constitutively expressed by a variety of lung-resident cells, including the epithelium, alveolar macrophages, and fibroblasts ${ }^{41-43}$. Indeed, we found that lung tissue had the highest level of TGF $\beta 1$ compared to the gut and pancreas under physiological conditions, which is in line with the lung-restricted Nrp1 expression. Previous studies have shown that TGF $\beta 1$ signaling plays critical roles in modulating immunity. TGF $\beta 1$ can regulate not only $\mathrm{T}$ cell proliferation and differentiation, but also ILC differentiation and function ${ }^{41,44-46}$. One study showed that TGF $\beta 1$ signaling can program the development and maturation of ILC2s from their bone marrow progenitors, because deficiency of TGF $\beta$ receptor II resulted in the defective generation of ILC2 $\mathrm{s}^{46}$. Given that Nrp1 is specifically expressed on mature lung ILC2s, but not ILC2 progenitors in bone marrow, we can rule out the possibility of Nrp1 involvement in the effect of TGF $\beta 1$ on ILC2 differentiation. Another study found that pulmonary epithelial-cell-derived TGF $\beta 1$ can enhance ILC2 migration and production of type 2 cytokines via TGF $\beta$ RII, and therefore can promote allergic lung inflammation ${ }^{41}$. Our data support the finding that TGF $\beta 1$ can enhance ILC2s activation, but provide additional evidence for the role of $\mathrm{Nrp} 1$ in mediating TGF $\beta 1$ signaling regulating ILC2 function. Taken together, these data suggest that engagement of both Nrp1 and TGF $\beta$ RII is required for TGF $\beta 1$-induced ILC2 activation.

Nrp1-deficient ILC2s did not exhibit any functional defects in the steady state. However, they displayed decreased production of type 2 cytokines under the disease conditions of bleomycin-induced fibrosis and $N$. brasiliensis infection. These data indicate that disease-specific signals are required to activate the downstream pathways of Nrp1. Despite the high expression level in steady state, we found that mice displayed a striking increase of TGF $\beta 1$ in lung tissue under pathological conditions. Lung epithelial cells are considered the major resource of TGF $\beta 1$ (refs. ${ }^{42,47}$ ). Upon exposure to bleomycin, lung epithelial cells release large amounts of both TGF $\beta 1$ and IL-33. IL-33 can directly induce TGF $\beta 1$ release by lung epithelium ${ }^{41}$, which promotes the accumulation of TGF $\beta 1$ in lung and leads to enhanced ILC2 activation via Nrp1. Together, these data suggest that Nrp1 serves as a lung-specific modulator for ILC2 activation.

RNA-seq analysis revealed that ST2 was downregulated in Nrp1-deficient ILC2s from bleomycin-challenged mice. As the IL-33 receptor, ST2 directly affects the magnitude of type 2 immune responses. Thus, the impairment of ILC2 function in Nrp1-deficient mice was presumably due decreased ST2 expression. Our in vitro data showed that TGF $\beta 1$ promoted ST2 expression on sorted lung ILC2s, whereas ablation of Nrp1 abolished the upregulation of ST2 induced by TGF $\beta 1$. In accord with our findings, the pathogenic role of IL-33/ST2 signaling and ILC2 in lung fibrosis has been reported in previous studies ${ }^{32,33}$. Our data from the bleomycin-induced fibrosis model indicate that the TGF $\beta 1-N r p 1$ axis enhances ILC2 function and exacerbates lung fibrosis through upregulation of ST2. One study found that TGF $\beta 1$-induced ST2 expression in BM ILC2 progenitors is mediated by the MEK1/2-dependent pathway ${ }^{46}$. Given the lack of Nrp1 expression in BM ILC2 progenitors, we speculate that a different downstream TGF $\beta 1$ signaling pathway for ST2 induction is involved.

Genetic ablation or pharmacological inhibition of Nrp1 suppressed ILC2 function and protected mice from the development of pulmonary fibrosis. Moreover, NRP1 inhibition also resulted in reduced activation of human lung ILC2s. This establishes a strong rationale for the translation of our mouse studies into human diseases. These data raise the possibility that targeting NRP1 may represent a promising approach to suppress ILC2 activation in the lung while leaving ILC2 function intact in other tissues. Notably, NRP1 is expressed in a variety of immune cells and plays multiple roles in these cells. Thus, targeting NRP1 could affect the host immune response in a complex manner. However, our data showed that the efficacy achieved by pharmacological inhibition of Nrp1 was dependent on ILC2s. Studies have shown that NRP1 is also expressed on lung epithelial cells and is required for severe acute respiratory syndrome coronavirus 2 (SARS-CoV-2) entry ${ }^{40,48}$. Blockade of NRP1 could reduce the entry of SARS-CoV-2 in cell culture. Because pulmonary fibrosis is a common complication of COVID-19, we speculate that NRP1 would be a potent therapeutic target for COVID-19 through its dual mechanism of action. Thus, NRP1 is a potential therapeutic target for a wide range of ILC2-based lung inflammatory diseases, such as fibrosis and allergic airway disease. Our findings provide insights into how tissue-resident immune cells integrate into local environmental niches to regulate tissue-specific immunity and reveal that targeting the tissue-specific signature is a novel strategy for precision medicine.

\section{Online content}

Any methods, additional references, Nature Research reporting summaries, source data, extended data, supplementary information, acknowledgements, peer review information; details of author contributions and competing interests; and statements of data and code availability are available at https://doi.org/10.1038/ s41590-021-01097-8.

Received: 30 March 2021; Accepted: 18 November 2021; Published online: 24 January 2022

\section{References}

1. Hoyler, T. et al. The transcription factor GATA3 controls cell fate and maintenance of type 2 innate lymphoid cells. Immunity 37, 634-648 (2012)

2. Kim, B. S. et al. TSLP Elicits IL-33-Independent innate lymphoid cell responses to promote skin inflammation. Sci. Transl. Med. 5, 170 (2013).

3. Gasteiger, G., Fan, X., Dikiy, S., Lee, S. Y. \& Rudensky, A. Y. Tissue residency of innate lymphoid cells in lymphoid and nonlymphoid organs. Science 350, 981-985 (2015).

4. Ricardo-Gonzalez, R. R. et al. Tissue signals imprint ILC2 identity with anticipatory function. Nat. Immunol. 19, 1093-1099 (2018).

5. von Moltke, J., Ji, M., Liang, H.-E. \& Locksley, R. M. Tuft-cell-derived IL-25 regulates an intestinal ILC2-epithelial response circuit. Nature 529, 221-225 (2016).

6. Dahlgren, M. W. et al. Adventitial stromal cells define group 2 innate lymphoid cell tissue niches. Immunity 50, 707-722 (2019).

7. Cardoso, V. et al. Neuronal regulation of type 2 innate lymphoid cells via neuromedin U. Nature 549, 277-281 (2017).

8. Schneider, C. et al. Tissue-resident group 2 Innate lymphoid cells differentiate by layered ontogeny and in situ perinatal priming. Immunity 50, 1425-1438 (2019).

9. Robinette, M. L. et al. Transcriptional programs define molecular characteristics of innate lymphoid cell classes and subsets. Nat. Immunol. 16, 306-317 (2015)

10. Simoni, Y. et al. Human innate lymphoid cell subsets possess tissue-type based heterogeneity in phenotype and frequency. Immunity 46, 148-161 (2017). 
11. Ricardo-Gonzalez, R. R. et al. Tissue-specific pathways extrude activated ILC2s to disseminate type 2 immunity. J. Exp. Med. 217, e20191172 (2020).

12. Yudanin, N. A. et al. Spatial and temporal mapping of human innate lymphoid cells reveals elements of tissue specificity. Immunity 50, 505-519 (2019).

13. Rana, B. M. J. et al. A stromal cell niche sustains ILC2-mediated type-2 conditioning in adipose tissue. J. Exp. Med. 216, 1999-2009 (2019).

14. Martinez-Gonzalez, I. et al. Allergen-experienced group 2 innate lymphoid cells acquire memory-like properties and enhance allergic lung inflammation. Immunity 45, 198-208 (2016).

15. Huang, Y. et al. S1P-dependent interorgan trafficking of group 2 innate lymphoid cells supports host defense. Science 359, 114-119 (2018).

16. Huang, Y. et al. IL-25-responsive, lineage-negative KLRG ${ }^{\text {hi }}$ cells are multipotential 'inflammatory' type 2 innate lymphoid cells. Nat. Immunol. 16, 161-169 (2015).

17. Evren, E. et al. Distinct developmental pathways from blood monocytes generate human lung macrophage diversity. Immunity 54, 259-275 (2021)

18. Seidman, J. S. et al. Niche-specific reprogramming of epigenetic landscapes drives myeloid cell diversity in nonalcoholic steatohepatitis. Immunity 52, 1057-1074 (2020).

19. Qiu, J. et al. Tissue signals imprint Aiolos expression in ILC2s to modulate type 2 immunity. Mucosal Immunol. 14, 1306-1322 (2021).

20. Kolodkin, A. L. et al. Neuropilin is a semaphorin III receptor. Cell $\mathbf{9 0}$, 753-762 (1997).

21. Soker, S., Takashima, S., Miao, H. Q., Neufeld, G. \& Klagsbrun, M. Neuropilin-1 is expressed by endothelial and tumor cells as an isoform-specific receptor for vascular endothelial growth factor. Cell $\mathbf{9 2}$, 735-745 (1998).

22. Glinka, Y. \& Prud'homme, G. J. Neuropilin-1 is a receptor for transforming growth factor beta-1, activates its latent form, and promotes regulatory $\mathrm{T}$ cell activity. J. Leukoc. Biol. 84, 302-310 (2008).

23. Delgoffe, G. M. et al. Stability and function of regulatory T cells is maintained by a neuropilin-1-semaphorin-4a axis. Nature 501, 252-256 (2013).

24. Leclerc, M. et al. Regulation of antitumour CD8 T-cell immunity and checkpoint blockade immunotherapy by neuropilin-1. Nat. Commun. 10, 3345 (2019).

25. Liu, C. et al. Neuropilin-1 is a T cell memory checkpoint limiting long-term antitumor immunity. Nat. Immunol. 21, 1010-1021 (2020).

26. Shikhagaie, M. M. et al. Neuropilin-1 Is expressed on lymphoid tissue residing LTi-like group 3 innate lymphoid cells and associated with ectopic lymphoid aggregates. Cell Rep. 18, 1761-1773 (2017).

27. Heldin, C. H., Miyazono, K. \& tenDijke, P. TGF- $\beta$ signalling from cell membrane to nucleus through SMAD proteins. Nature 390, 465-471 (1997).

28. Seillet, C. et al. Deciphering the innate lymphoid cell transcriptional program. Cell Rep. 17, 436-447 (2016).

29. Miyazaki, M. et al. Id 2 and Id 3 maintain the regulatory T cell pool to suppress inflammatory disease. Nat. Immunol. 15, 767-776 (2014).

30. Hacker, C. et al. Transcriptional profiling identifies Id 2 function in dendritic cell development. Nat. Immunol. 4, 380-386 (2003).
31. Buitenhuis, M. et al. Differential regulation of granulopoiesis by the basic helix-loop-helix transcriptional inhibitors Id1 and Id2. Blood 105, 4272-4281 (2005).

32. Li, D. et al. IL-33 promotes ST2-dependent lung fibrosis by the induction of alternatively activated macrophages and innate lymphoid cells in mice. J. Allergy Clin. Immunol. 134, 1422-1432 (2014).

33. Hams, E. et al. IL-25 and type 2 innate lymphoid cells induce pulmonary fibrosis. Proc. Natl Acad. Sci. USA 111, 367-372 (2014).

34. $\mathrm{Bi}$, J. et al. NK cells alleviate lung inflammation by negatively regulating group 2 innate lymphoid cells. J. Immunol. 198, 3336-3344 (2017).

35. Meng, X.-m, Nikolic-Paterson, D. J. \& Lan, H. Y. TGF- $\beta$ : the master regulator of fibrosis. Nat. Rev. Nephrol. 12, 325-338 (2016).

36. Nussbaum, J. C. et al. Type 2 innate lymphoid cells control eosinophil homeostasis. Nature 502, 245-248 (2013).

37. Passalacqua, G. et al. IL-13 and idiopathic pulmonary fibrosis: Possible links and new therapeutic strategies. Pulm. Pharmacol. Therapeutics 45, 95-100 (2017).

38. Lichtman, M. K., Otero-Vinas, M. \& Falanga, V. Transforming growth factor beta (TGF- $\beta$ ) isoforms in wound healing and fibrosis. Wound Repair Regeneration 24, 215-222 (2016)

39. Gieseck, R. L. 3rd, Wilson, M. S. \& Wynn, T. A. Type 2 immunity in tissue repair and fibrosis. Nat. Rev. Immunol. 18, 62-76 (2018).

40. Daly, J. L. et al. Neuropilin-1 is a host factor for SARS-CoV-2 infection. Science 370, 861-865 (2020).

41. Denney, L. et al. Pulmonary epithelial cell-derived cytokine TGF- $\beta 1$ Is a critical cofactor for enhanced innate lymphoid cell function. Immunity 43, 945-958 (2015)

42. de Boer, W. I. et al. Transforming growth factor beta(1) and recruitment of macrophages and mast cells in airways in chronic obstructive pulmonary disease. Am. J. Respiratory Crit. Care Med. 158, 1951-1957 (1998).

43. Sullivan, D. E., Ferris, M., Nguyen, H., Abboud, E. \& Brody, A. R. TNF- $\alpha$ induces TGF- $\beta(1)$ expression in lung fibroblasts at the transcriptional level via AP-1 activation. J. Cell. Mol. Med. 13, 1866-1876 (2009).

44. Jones, C. P., Gregory, L. G., Causton, B., Campbell, G. A. \& Lloyd, C. M. Activin A and TGF- $\beta$ promote $\mathrm{T}(\mathrm{H}) 9$ cell-mediated pulmonary allergic pathology. J. Allergy Clin. Immunol. 129, 1000-1010 (2012).

45. Laouar, Y., Sutterwala, F. S., Gorelik, L. \& Flavell, R. A. Transforming growth factor-beta controls $\mathrm{T}$ helper type 1 cell development through regulation of natural killer cell interferon-gamma. Nat. Immunol. 6, 600-607 (2005).

46. Wang, L. et al. TGF- $\beta$ induces ST2 and programs ILC2 development. Nat. Commun. 11, 35 (2020).

47. Khalil, N., O’Connor, R. N., Flanders, K. C. \& Unruh, H. TGF- $\beta-1$, but not TGF- $\beta-2$ or TGF- $\beta-3$, is differentially present in epithelial cells of advanced pulmonary fibrosis: An immunohistochemical study. Am. J. Respir. Cell Mol. Biol. 14, 131-138 (1996)

48. Cantuti-Castelvetri, L. et al. Neuropilin-1 facilitates SARS-CoV-2 cell entry and infectivity. Science 370, 856-860 (2020).

Publisher's note Springer Nature remains neutral with regard to jurisdictional claims in published maps and institutional affiliations.

(c) The Author(s), under exclusive licence to Springer Nature America, Inc. 2022 


\section{Methods}

Mice. Wild-type mice, including those with C57BL/6, BALB/c, DBA, ICR, and S129 backgrounds, were purchased from Shanghai Laboratory Animal Center

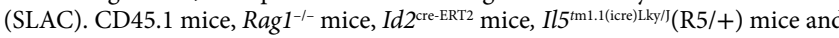

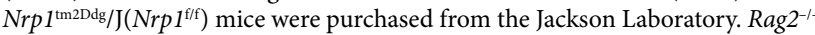
Il $\mathrm{rg}^{-1-}$ mice were purchased from Taconic Biosciences. All mice were bred and maintained at accredited animal facilities under specific-pathogen-free conditions in individually ventilated cages on a strict 12-hour day-night cycle with a regular chow diet. Mice used in this study were 6-8 weeks old and sex-matched, unless otherwise indicated in the text. Six-week-old $I d 2^{\text {cre-ERT2 }} N r p 1^{\mathrm{f} /+}$ and $I d 2^{\text {cre-ERT2 }} N r p 1^{\mathrm{f} / \mathrm{f}}$ mice were injected intraperitoneally with tamoxifen ( $2 \mathrm{mg}$ /day per mouse) for 5 days to knock down Nrp1 expression in the ILC lineage, and other treatments were performed 3 days later. Littermate mice were randomly grouped into control and treatment groups for all experiments in this study. All animal experiments were performed in compliance with the Guide for the Care and Use of Laboratory Animals, approved by Shanghai Jiao Tong University School of Medicine Institutional Animal Care and Use Committees (IACUC) and performed according to guidelines for the use and care of laboratory animals as provided by Shanghai Jiao Tong University School of Medicine Institutional Animal Care and Use Committees (IACUC)

Bleomycin-induced lung fibrosis. Mice were lightly anesthetized with isoflurane gas (4\%) first. Then, bleomycin sulfate $(2 \mathrm{mg} / \mathrm{kg}$ body weight, $30 \mu \mathrm{l}$ per mouse, APExBio) or PBS control was administered intranasally. BALF and lungs were collected 21 days later or at the indicated time points and subjected to downstream analysis. For EG00229 treatment, mice were intraperitoneally $(10 \mathrm{mg} / \mathrm{kg}$ body weight, $100 \mu \mathrm{l} /$ mouse $)$ or intranasally $(2.5 \mathrm{mg} / \mathrm{kg}$ body weight, $20 \mu \mathrm{l} /$ mouse $)$ injected with EG00229 (APExBio) 3 times weekly for 3 weeks after administration of bleomycin. For anti-Thyl.2 antibody treatment, mice were intraperitoneally injected with anti-Thy1.2 (200 $\mu$ g per mouse in $100 \mu$ l of PBS, BioXCell) or IgG1 isotype control antibody every 3 days after administration of bleomycin. To collect BALF, $0.8 \mathrm{ml}$ of ice-cold PBS was injected into the mouse trachea with a 22-G needle. After instillation and withdrawal 3 times, supernatant was collected by centrifugation at $400 \mathrm{~g}$ for 5 minutes at $4^{\circ} \mathrm{C}$.

Nippostrongylus brasiliensis infection. Mice were inoculated subcutaneously with 500 third-stage $N$. brasiliensis larvae (L3). Lung tissues were collected and analyzed on day 5 . The worm burden in small intestine was measured on day 7 .

Cell preparation from tissues. Lung or pancreas was cut into pieces and digested in $5 \mathrm{ml}$ of RPMI 1640 medium (Thermo Fisher Scientific) containing DNase I $(750 \mathrm{~g} / \mathrm{ml}$ for digestion of lung (pancreas was digested without DNase I), Sigma-Aldrich) and collagenase VIII ( $200 \mathrm{U} / \mathrm{ml}$, Sigma-Aldrich) at $37^{\circ} \mathrm{C}$ for 1 hour (for digestion of lung) or 30 minutes (for digestion of pancreas). The digested tissues were passed through a $70-\mu \mathrm{m}$ cell strainer after vigorous shaking. Mononuclear cells were then collected from the interphase of a $40 \%$ and $80 \%$ Percoll (GE Healthcare) gradient following centrifugation at 2,500 r.p.m. for 20 minutes at room temperature.

When fat tissues and Peyer's patches were removed, the large intestine and small intestine were cut into pieces and washed in PBS. Intestines were incubated and shaken at 220 r.p.m. in PBS containing $1 \mathrm{mM}$ dithiothreitol (Sigma) for 10 minutes and then in PBS containing $30 \mathrm{mM}$ EDTA (Sangon Biotech) at $37^{\circ} \mathrm{C}$ for 10 minutes for 2 cycles. Tissues were digested in RPMI 1640 medium containing DNase I $(150 \mu \mathrm{g} / \mathrm{ml})$ and collagenase VIII $(150 \mathrm{U} / \mathrm{ml})$ at $37^{\circ} \mathrm{C}$ in an incubator with $5 \% \mathrm{CO}_{2}$ for 1.5 hour. Intestinal mononuclear cells were collected according to the protocols above.

Bone marrow cells were processed by flushing femur and tibia with a syringe. Red blood cells were removed with $3 \mathrm{ml}$ of ACK Lysing Buffer (Gibco) at $4^{\circ} \mathrm{C}$ for 3 minutes and centrifuged at $400 \mathrm{~g}$ for 5 minutes.

Isolation of mononuclear cells from human blood, BALF, and lung. Samples were collected from people with non-small cell lung cancer (NSCLC) or NSCLC with idiopathic pulmonary fibrosis (IPF). Bronchoalveolar lavage fluid and tumor-adjacent lung tissues were retrieved at the Shanghai Chest Hospital following the study protocol under approval KS(Y)21172. Informed consent was obtained from all individuals. The study was performed in strict compliance with all institutional ethical regulations. Participant characteristics are detailed in Supplementary Table 1

Human peripheral blood was mixed with an equal volume of PBS and then mononuclear cells were collected from the interphase of Lymphoprep (Stem Cell) gradient after spinning at $400 \mathrm{~g}$ for 30 minutes at room temperature.

Human BALF was diluted with PBS and then passed through a $70-\mu \mathrm{m}$ cell strainer. The cell suspension was collected after spinning at $800 \mathrm{~g}$ for 10 minutes at room temperature.

Human lung samples were cut into pieces and digested in RPMI 1640 medium containing DNase I $(150 \mu \mathrm{g} / \mathrm{ml})$ and collagenase VIII $(300 \mathrm{U} / \mathrm{ml})$ at $37^{\circ} \mathrm{C}$ for 1 hour. The digested tissues were passed through a $70-\mu \mathrm{m}$ cell strainer after vigorous shaking. To enrich lung lymphocytes, lung cells were resuspended in $4 \mathrm{ml}$ of RPMI 1640 medium and loaded onto the top of Lymphoprep layer. Human lung mononuclear cells were collected after centrifugation at $400 \mathrm{~g}$ for 30 minutes at room temperature.

Flow cytometry and cell sorting. Dead cells were stained with fixable viability stain 520 (BD Biosciences) and removed. CD16/32 antibody (Biolegend) was used to block nonspecific binding to $\mathrm{Fc}$ receptors before surface staining. For surface staining, cells were resuspended in $100 \mu \mathrm{l}$ of FACS buffer (BD Biosciences) containing antibody cocktails and stained at $4^{\circ} \mathrm{C}$ in the dark for 30 minutes. Antibodies used for flow cytometry are listed in Supplementary Table 2. For intracellular staining, cells were fixed and permeabilized by Foxp3 Fixation/ Permeabilization kit (Thermo Fisher Scientific) at $4^{\circ} \mathrm{C}$ for 45 minutes and stained with $100 \mu \mathrm{l}$ of $1 \times$ permeabilization buffer containing antibody cocktails at $4{ }^{\circ} \mathrm{C}$ in the dark for 45 minutes. For cytokine staining, cells were stimulated with PMA (50 ng/ml, Sigma-Aldrich) and ionomycin ( $500 \mathrm{ng} / \mathrm{ml}$, Sigma-Aldrich) for 4 hours, and brefeldin A ( $2 \mu \mathrm{g} / \mathrm{ml}$, Sigma-Aldrich) was added for the last 2 hours before cells were collected for analysis. For phosphorylation staining, cells were fixed by $1 \times \mathrm{BD}$ Phosflow Lyse/Fix Buffer (BD Biosciences) at $37^{\circ} \mathrm{C}$ for 10 minutes, permeabilized by BD Phosflow Perm Buffer III at $4^{\circ} \mathrm{C}$ for 30 minutes, and then stained with phosphorylated antibodies at room temperature for 30 minutes. For cell sorting, ILC2s from lung, pancreas, large intestine were sorted as lin $^{-}\left(\mathrm{CD}^{-} \mathrm{B} 220^{-} \mathrm{CD}^{-} \mathrm{CD} 11 \mathrm{~b}^{-} \mathrm{CD} 11 \mathrm{c}^{-}\right) \mathrm{CD} 5^{+} \mathrm{ST} 2^{+} \mathrm{CD} 25^{+}$live cells or $\mathrm{lin}^{-} \mathrm{CD} 45^{+} \mathrm{ST} 2^{+} \mathrm{R}^{+}$live cells (in R5/+ mice and R5/+Nrp1 $1^{\mathrm{fff}}$ mice), and ILC2s from small intestine were sorted as $\operatorname{lin}^{-} \mathrm{CD} 45^{\text {hi }} \mathrm{CD} 90^{\mathrm{lo}} \mathrm{KLRG} 1^{+}$live cells. Human ILC2s were sorted as lin $^{-}\left(\mathrm{CD} 1 \mathrm{a}^{-} \mathrm{CD} 3^{-} \mathrm{CD} 5^{-} \mathrm{CD} 11 \mathrm{~b}^{-} \mathrm{CD} 11 \mathrm{c}^{-} \mathrm{CD} 14^{-} \mathrm{CD} 15^{-} \mathrm{CD} 16^{-} \mathrm{CD} 19^{-} \mathrm{F}\right.$ ceR1 $\left.1^{-}\right) \mathrm{CD} 45^{+} \mathrm{CD} 127^{+} \mathrm{CRTH} 2^{+}$live cells.

All flow data were acquired by BD FACSDiva software v8.0.2 and analyzed by FlowJo VX. $t$-SNE analysis parameters included: FJComp-APC-PD-1, FJComp-AF700-CD45, FJComp-FITC- fixable viability stain, FJComp-PerCP eFluor-CCR9, FJComp-BB790-CD69, FJComp-BUV395-GATA3, FJComp-BUV661-CD25, FJComp-PE-KLRG1, FJComp-PECy5-CD127, FJComp-PECy7-Nrp1, FJComp-PECF594-Lin, FJComp-BV421-ST2, FJComp-BV605-IL-17RB, FJComp-BV650-SCA1, FJComp-BV750-MHC II, Iterations-1000, Perplexity-20, Eta(learning rate)-200.

Adoptive transfer. ILC2s from lung, large intestine, and small intestine were sorted by BD FACSAria III. Then, 20,000 ILC2s from each tissue were transferred

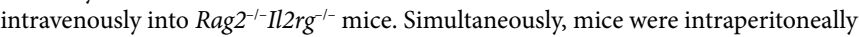
injected with $500 \mathrm{ng}$ of IL-33 for 4 consecutive days to expand ILC2s. Mice were euthanized for analysis after 3 weeks.

To confirm the cell-intrinsic effect of Nrp1 in ILC2s, R5/+ (WT) and $\mathrm{R} 5 /+N r p 1^{\text {fff }}(\mathrm{KO})$ mice were injected with $500 \mathrm{ng}$ of IL-33 for 4 consecutive days to expand ILC2 compartments. Then $2 \times 10^{5}$ ILC2s sorted from WT or KO mice were

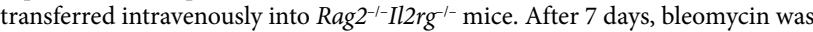
administered intranasally to the recipient mice to induce pulmonary fibrosis.

ILC2 culture. Sorted ILC2s were cultured in 96-well flat-bottom plates in RPMI 1640 complete medium containing 10\% FBS (Gibco), $2 \mathrm{mM}$ glutamine (Gibco), $50 \mu \mathrm{M}$ of $\beta$-mercaptoethanol, $333 \mathrm{U} / \mathrm{ml}$ of penicillin (Gibco), and $333 \mu \mathrm{g} / \mathrm{ml}$ of streptomycin (Gibco), in the presence of $10 \mathrm{ng} / \mathrm{ml} \mathrm{IL-7} \mathrm{(Peprotech).} \mathrm{For}$ stimulation with different ligands, sorted ILC2s from large intestine were cultured for 48 hours in the presence of IL-33 (10 ng/ml), TGF $\beta 1(1 \mathrm{ng} / \mathrm{ml})$, VEGF164 $(100 \mathrm{ng} / \mathrm{ml})$, or Sema3a $(100 \mathrm{ng} / \mathrm{ml})$, respectively. For inhibitor treatment, sorted ILC2s from large intestine were cultured for 48 hours in the presence of TGF $\beta 1$ ( $1 \mathrm{ng} / \mathrm{ml}$ ) with SB431542 (1 $\mu \mathrm{M}$, APExBio), LY2109761 (5 $\mu \mathrm{M}$, Selleck), SIS3 HCl ( $2 \mu \mathrm{M}$, Selleck), or DMSO control. To induce ST2 in WT and KO ILC2s, ILC2s were cultured at 10,000 cells per well with $1 \mathrm{ng} / \mathrm{ml}$ of mouse TGF $\beta 1$ (Biolegend) or with different concentrations of TGF $\beta 1$ for 2 hours or 48 hours, and then cells were collected for flow cytometry analysis. For human ILC2 culture, isolated ILC2s were plated at 5,000 cells per well in RPMI 1640 complete medium containing $20 \mathrm{ng} / \mathrm{ml}$ human IL-7 (Peprotech). For induction of Nrp1 on human ILC2s, blood ILC2s were cultured with $1 \mathrm{ng} / \mathrm{ml}$ of human TGF $\beta 1$ (R\&D Systems) for 48 hours. For in vitro ILC2s activation, mouse ILC2s or human total lung mononuclear cells were pretreated with TGF $\beta 1$ at $1 \mathrm{ng} / \mathrm{ml}$ for 24 hours and then stimulated with IL-33 at $10 \mathrm{ng} / \mathrm{ml}$ for another 24 hours. IL-13 in the supernatants was detected by ELISA. The percentage of IL- $13^{+}$or IL-5 $5^{+}$ILC2s was determined by flow cytometry. For Nrp1 inhibition experiments, human lung mononuclear cells were treated with the small-molecule Nrp1 inhibitor EG00229 at $10 \mu \mathrm{M}$ for 48 hours.

Mononuclear cells co-culture. For mononuclear cells co-culture, mononuclear cells isolated from the indicated tissues identified with separate congenic leukocyte markers were cultured in 48-well plates in RPMI 1640 complete medium for 24 hours or 48 hours. For TGF $\beta 1$ blockade experiments, mononuclear cells were treated with anti-TGF $\beta 1$ antibody $(5 \mu \mathrm{g} / \mathrm{ml}, \mathrm{R} \& \mathrm{D}$ Systems) or isotype control IgG1 antibody ( $5 \mu \mathrm{g} / \mathrm{ml}$, Biolegend) for 48 hours, and then cells were collected for flow cytometry.

qRT-PCR. RNA was isolated with TRIzol reagent (Invitrogen). cDNA was synthesized using PrimeScriptRT master Kit (Takara). qRT-PCR was performed by using SYBRSelect Master Mix (Life-ABI), and reactions were run with 
QuantStudio Real-Time PCR software V1.3 (Thermo Fisher Scientific). The results are displayed as relative expression values normalized to Actb. Primers used for qRT-PCR are listed in Supplementary Table 3

Cytokine measurement. The concentration of bioactive TGF $\beta 1$ in BALF and lung tissue lysates was tested by TGF $\beta 1$ Quantikine ELISA Kit (R\&D System), and the concentration of IL-13 in the cell culture supernatants was tested by IL-13 mouse ELISA Kit (Thermo Fisher Scientific), according to the manufacturer's instructions.

Immunoblot analysis. Tissue was lysed in RIPA buffer (Beyotime) containing protein inhibitors (Thermo Fisher Scientific). Protein concentration was determined by BCA protein assay (Beijing Boling Kewei Biotechnology). Proteins were mixed with SDS Page loading buffer (Beyotime) and incubated at $100^{\circ} \mathrm{C}$ for 10 minutes. Then, $40 \mu \mathrm{g}$ of protein lysate per lane was run through $10 \%$ Tris-Glycine Gels and transferred to Immobilon-P Transfer Membrane (Merck Millipore). The membrane was blocked for 1 hour in $5 \%$ nonfat dried milk in Tris-buffered saline containing $0.1 \%$ Tween 20 (TBST) and incubated overnight with primary antibody at $4{ }^{\circ} \mathrm{C}$. The membrane was then washed 3 times in TBST and incubated with an HRP-conjugated secondary antibody for 1 hour at room temperature. Detection was performed with ECL western blotting detection reagents (GE Healthcare). Antibodies used in the study are listed in Supplementary Table 2.

Histology. The largest left lung lobe was excised, fixed in 4\% paraformaldehyde (Sangon Biotech), and embedded in paraffin. Sections were stained with H\&E or Gomori Rapid One-Step Trichrome Stain for collagen and then were examined by light microscopy. Images were acquired using OLYMPUS BX53 system and cellSens Standard software.

RNA sequencing. Total RNA from sorted lung ILC2s of R5/+ (WT) and $\mathrm{R} 5 /+\operatorname{Nrp} 1^{\mathrm{f} / \mathrm{f}}(\mathrm{KO})$ mice treated with bleomycin was extracted by TRIzol reagent. Each sample was pooled from 3-4 mice. RNA quality was examined with an Agilent Bioanalyzer 2100 (Agilent). The concentration of RNA was from $7 \mathrm{ng} / \mu \mathrm{l}$ to $14 \mathrm{ng} / \mu \mathrm{l}$. The SMART-Seq HT Kit (Takara Bio USA) was used to generate high-quality, full-length cDNA. Libraries were generated using TruePrep DNA Library Prep Kit V2 for Illumina (Vazyme) following the manufacturer's protocol The obtained libraries were sequenced on Illumina Hiseq X Ten system. The raw reads were aligned to the mouse reference genome (version mm10) by using Hisat2 RNA-seq alignment software. Quantitated relative mRNA expression levels (FPKM) were calculated on the basis of exon regions using Cufflinks and the $\mathrm{mm} 10$ reference genome annotations. Protein-coding genes were displayed in volcano plots and used for Gene Set Enrichment Analysis (Broad Institute). Differentially expressed genes identified by DESeq2 (fold change $>1.2, P<0.05$ ) were highlighted in the volcano plot and used for Gene Ontology (GO) analysis. Heat maps were generated from the $Z$-score by Hemi software. scRNA-seq data and human bulk RNA-seq data were obtained from Gene Expression Omnibus (GEO) and analyzed according to the reported paper.

Statistical analysis. For quantifications that were done with FACS, blinding was performed by labeling the test tubes numerically without prior knowledge of the genotype/treatment of the sample. The other samples were not blinded to the authors, since all measurements were objective and the conclusions were based on multiple independent experiments, technical replicates, or statistical significance. No data points were excluded from the analysis. Information about statistical details and methods is included in the figure legends. Unless otherwise noted, statistical analysis was performed with the two-tailed unpaired Student's $t$ test on individual biological samples using GraphPad Prism 8.0 program. Data from such experiments are presented as mean values \pm s.e.m. $P$ values are noted in each figure.

Reporting Summary. Further information on research design is available in the Nature Research Reporting Summary linked to this article.

\section{Data availability}

RNA-sequencing data have been deposited in GEO under the primary accession code GSE168809. Published ILC2 scRNA-seq datasets were accessed in GEO under the accession code GSE117568. Published ILC2 bulk RNA-seq dataset were accessed in GEO under the accession code GSE126107. The raw reads of RNA-seq were aligned to the mouse reference genome (version mm10). All other data are available in the article and supplementary files or from the corresponding authors upon reasonable request. Source data are provided with this paper.

\section{Acknowledgements}

We thank members of L. Shen's laboratory for their help and suggestions on this project. We thank the National Institute of Parasitic Diseases at Chinese Center for Disease Control and Prevention for help with $N$. brasiliensis infection experiments. We thank the Flow Cytometry Facility and Animal Facility at Shanghai Jiao Tong University School of Medicine for service and assistance. We thank J. Zhou and T. Hong for helping with human samples collection. We thank E. Engleman for editing the manuscript. This study was supported by grant 2020YFA0509200 (to L.S.) and 2020YFA0509103 (to J.Q.) from the Ministry of Science and Technology of China, grant 81971487 (to L.S.), 32022027 and 31970860 (to J.Q.) from the National Natural Science Foundation of China, grant 20ZR1430200, 20142202300 (to L.S.) and 20ZR1466900 (to J.Q.) from Science and Technology Commission of Shanghai Municipality, and Shanghai Jiao Tong University School of Medicine Innovation Team on Pediatric Research (to L.S.).

\section{Author contributions}

J.Z. and J.X.Q. performed the experiments and analyzed the data. W.Z. and X.H. contributed to human samples collection and experiment design. J.C. supervised worm infection experiments. W.M. and B.S. contributed to discussion. J.Z. performed bioinformatics analyses. L.S., J.Q., and J.Z. wrote the manuscript. L.S., J.Q., and B.H. conceived, designed, and supervised the project.

\section{Competing interests}

The authors declare no competing interests.

\section{Additional information}

Extended data is available for this paper at https://doi.org/10.1038/s41590-021-01097-8.

Supplementary information The online version contains supplementary material available at https://doi.org/10.1038/s41590-021-01097-8.

Correspondence and requests for materials should be addressed to Bin $\mathrm{He}$, Ju Qiu or Lei Shen.

Peer review information Nature Immunology thanks Ralph Stadhouders and the other, anonymous, reviewer(s) for their contribution to the peer review of this work. $\mathrm{N}$. Bernard was the primary editor on this article and managed its editorial process and peer review in collaboration with the rest of the editorial team.

Reprints and permissions information is available at www.nature.com/reprints. 
a

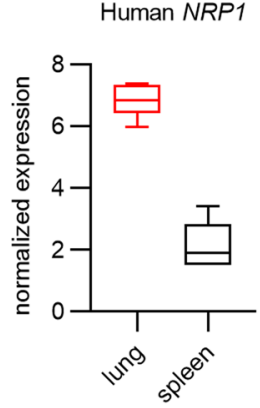

b

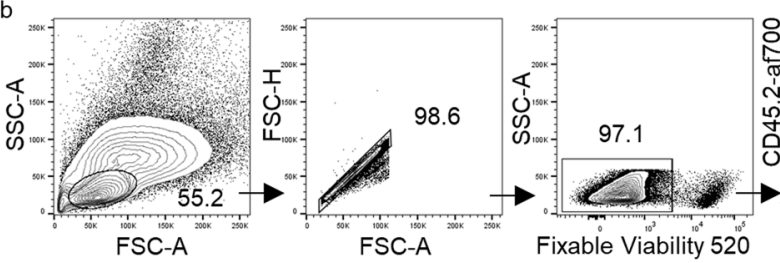

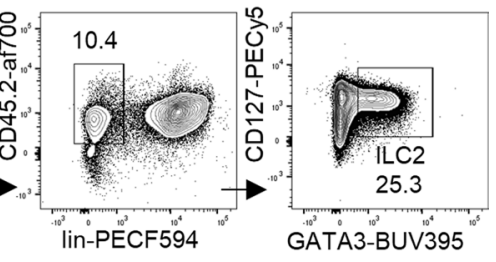

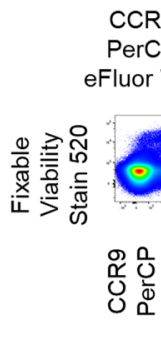

d

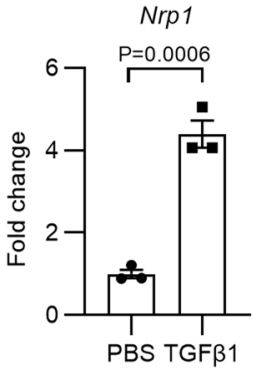

GATA3 CD25 lin

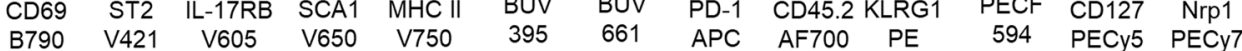
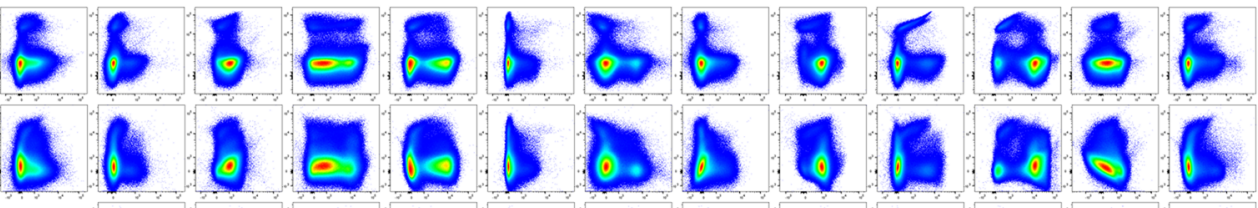

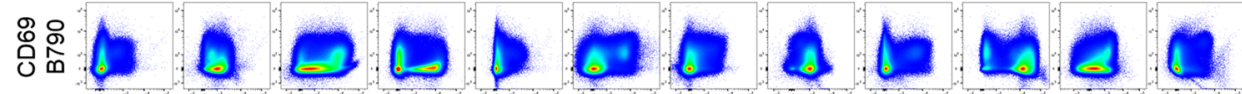
$\stackrel{\sim}{\stackrel{⿰}{*}}$

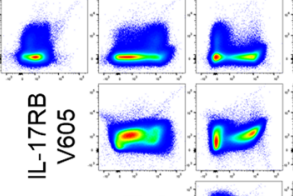

通员

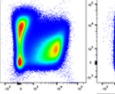

온용
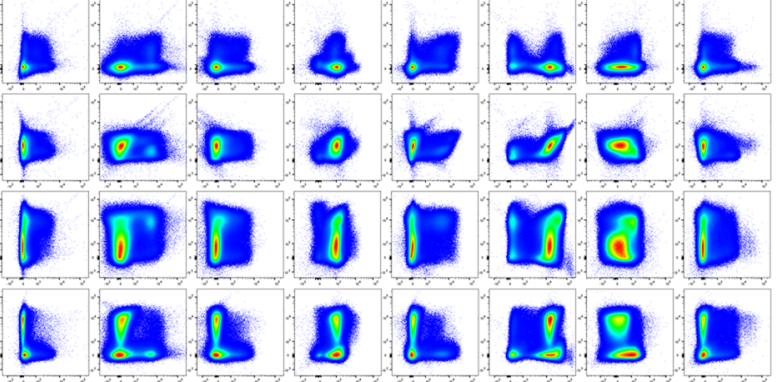

造》吕
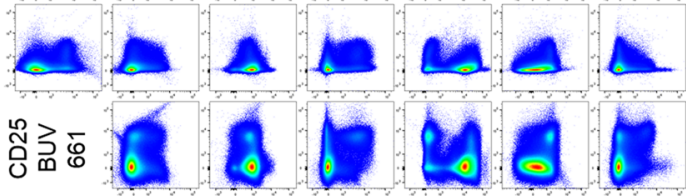

它虽

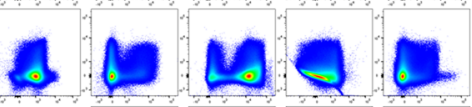

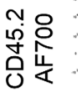

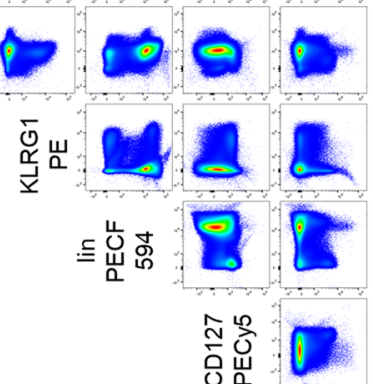

Extended Data Fig. 1 | Nrp1 is a tissue-specific marker of lung ILC2s. (a) Box plot compared the expression of NRP1 in human ILC2s from lung ( $n=6$ ) and spleen $(n=5)$. The bulk RNA-seq data were obtained from Gene Expression Omnibus (GEO) under accession code GSE126107. The center line, lower whisker, and upper whisker stand for median, minima, and maxima, respectively. Bounds of box are 25-75th percentile. (b) Gating strategy of ILC2s. ILC2s were gated on live and single cells with lin-(CD3-B220-CD11b-CD11c-CD5-) CD45+CD127+GATA3 ${ }^{+}$. (c) The compensation of 15-color (including 19 markers) flow cytometric panel was shown by $14 \times 14$ view. Cells were gated on single lymphocytes. (d) ILC2s sorted from large intestine were cultured with TGF $\beta 1$ at $1 \mathrm{ng} / \mathrm{ml}$ for $48 \mathrm{~h}$. mRNA expression level of Nrp1 was analyzed by quantitative RT-PCR ( $\mathrm{n}=3 / \mathrm{group}$ ). Each symbol represents an individual mouse (d). Data are representative of 3 independent experiments. Two-tailed unpaired Student's $t$ test. 

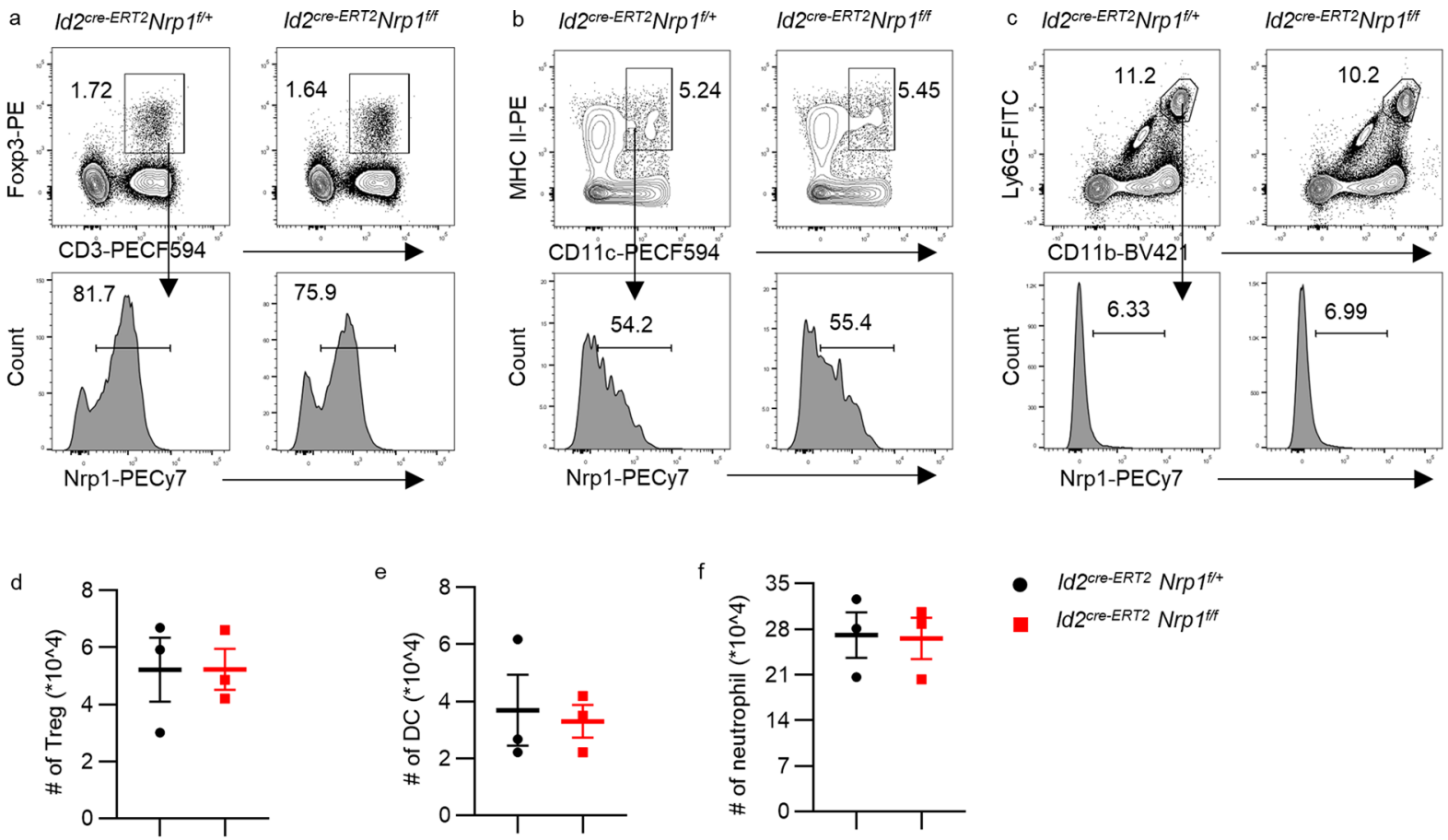

- Id $2^{\text {cre-ERT2 }} \mathrm{Nrp}^{\mathrm{ff+}}$
- Id $2^{\text {cre-ERT2 }} \mathrm{Nrp} 1^{f / f}$
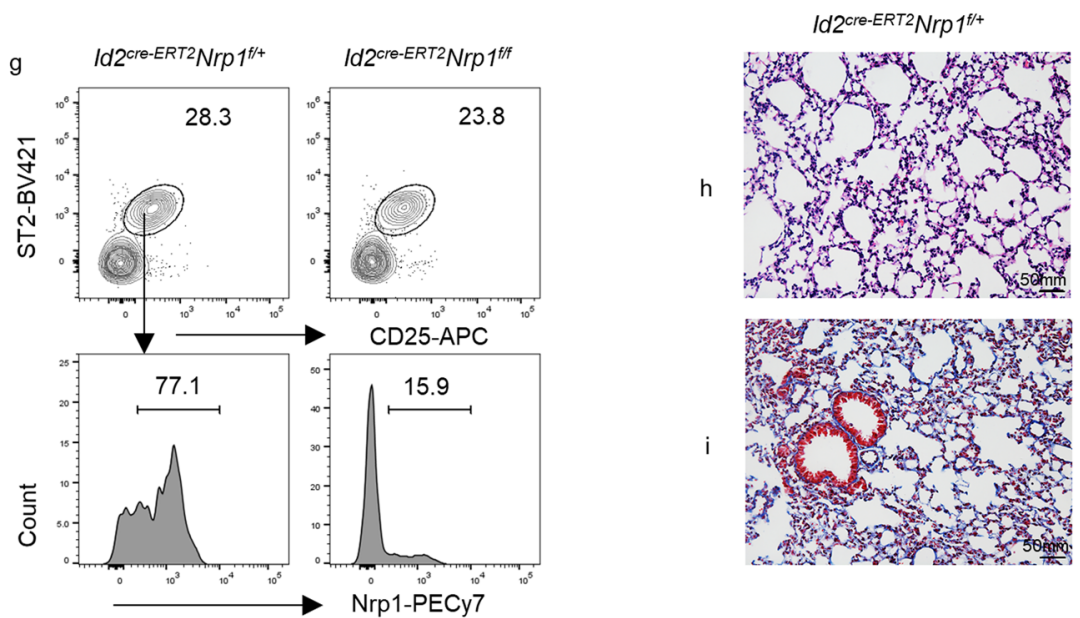

Id $2^{\text {cre-ERT2 }} \mathrm{Nrp}^{\text {fff }}$

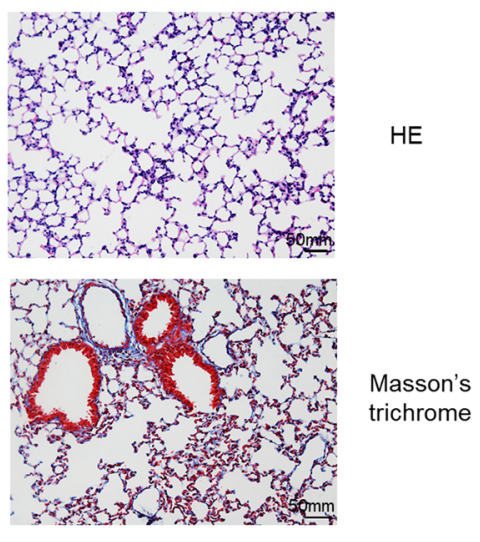

- Id $2^{\text {cre-ERT2 }} \mathrm{Nrp1} 1^{\mathrm{f} /+}$

- Id $2^{\text {cre-ERT2 }} \mathrm{Nrp} 1^{\mathrm{fit}}$

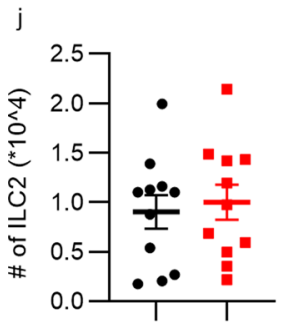

k
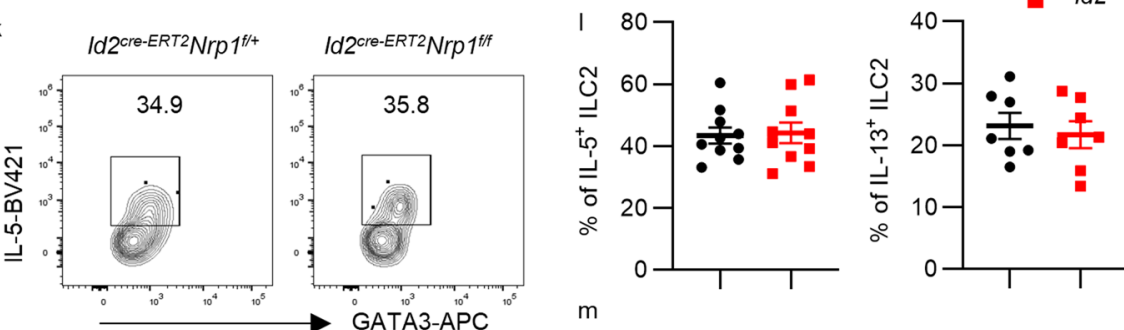

- Id $2^{\text {cre-ERT2 }} \mathrm{Nrp1} 1^{\mathrm{f} / \mathrm{+}}$
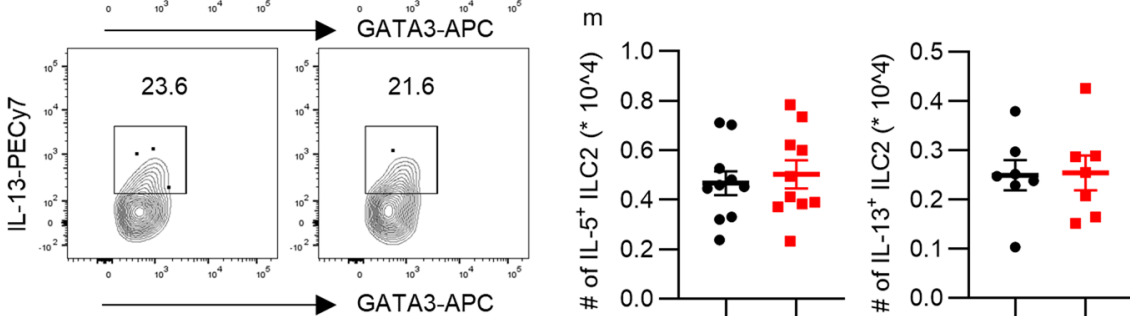

Extended Data Fig. 2 | See next page for caption. 


\section{NATURE IMMUNOLOGY}

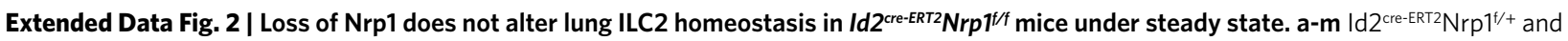
Id $2^{\text {cre-ERT2 }} \mathrm{Nrp}^{1 / f}$ mice were injected intraperitoneally with tamoxifen ( $2 \mathrm{mg} /$ day per mouse) for 5 days to knock out Nrp1 expression in the ILC lineage. (a-c) The expression level of Nrp1 on Treg, DCs, and neutrophils from lung were analyzed by flow cytometry. (a) The top row was gated on CD45+ live cells. (b) The top row was gated on CD45+CD11b+F4/80-Ly6G- live cells. (c) The top row was gated on CD45+ live cells. (d-f) Absolute cell counts of lung Treg, DCs, and neutrophils ( $n=3 /$ group). (g) The expression level of Nrp1 on lung ILC2s were analyzed by flow cytometry. Cells were gated on lin-CD45+CD127+ lymphocytes. (h, i) H\&E staining and Masson's trichrome staining of lung tissue. (j) Absolute cell counts of lung ILC2s ( $n=11 / g r o u p)$. (k) Percentage of IL-5+ ILC2s and IL-13+ ILC2s. Cells were gated on total ILC2s. (I, m) Frequency and total number of IL-5+ ILC2s $(n=10 / g r o u p)$ and $\mathrm{IL}-13^{+} \mathrm{ILC} 2 \mathrm{~s}(\mathrm{n}=7 /$ group) Each symbol represents an individual mouse ( $\mathbf{d}-\mathbf{f}, \mathbf{j}, \mathbf{l}$, and $\mathbf{m})$. Data are representative of 3-4 independent experiments and are presented as mean \pm SEM. 
a $\quad$ Id $2^{\text {cre-ERT2 }} \mathrm{Nrp} 1^{f /+}$

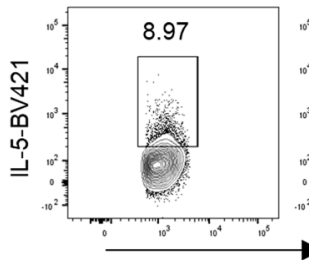

Id $2^{\text {cre-ERT2 }} \mathrm{Nrp} 1^{\text {fff }}$

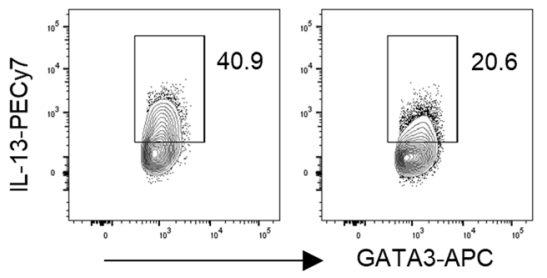

b

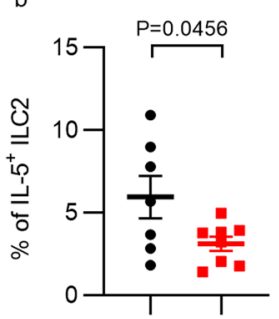

d

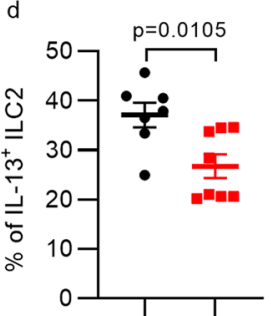

c
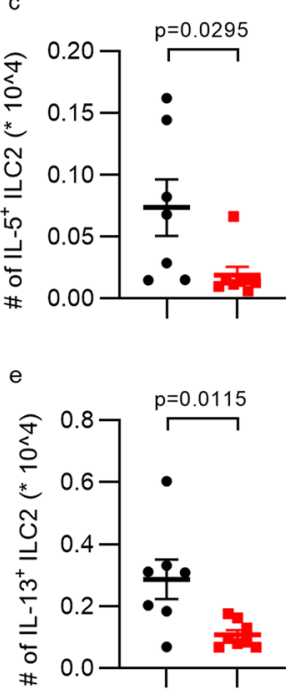

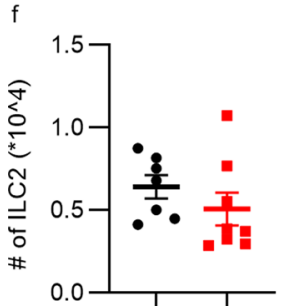

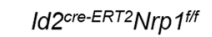

- Id $2^{\text {cre-ERT2 }} \mathrm{Nrp} 1^{\mathrm{f} / \mathrm{t}}$

- Id $2^{\text {cre-ERT2 }} \mathrm{Nrp} 1^{\mathrm{fff}}$
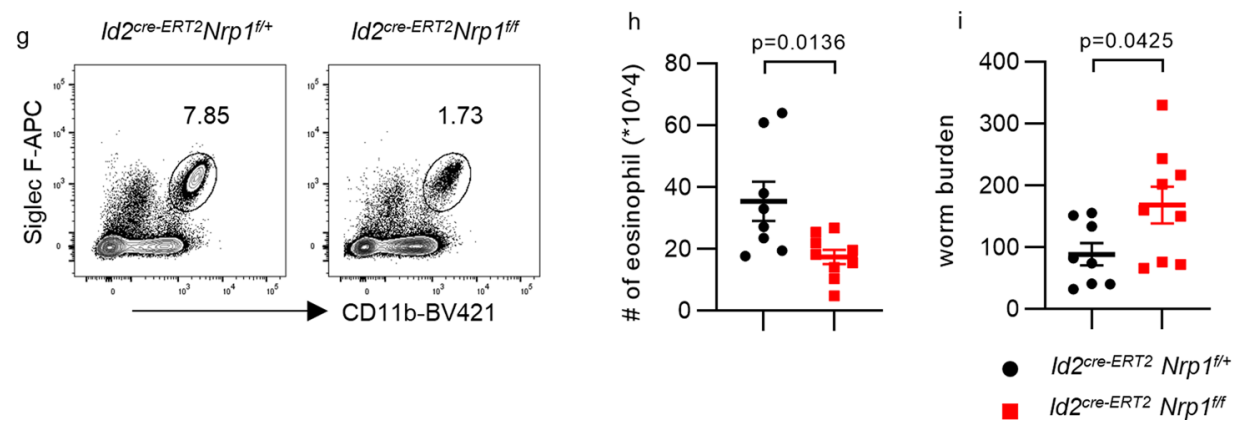

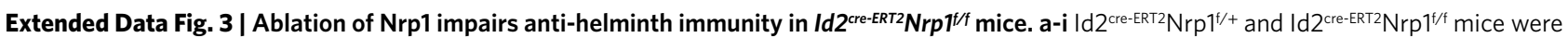
injected intraperitoneally with tamoxifen ( $2 \mathrm{mg} /$ day per mouse) for 5 days to knock out Nrp1 expression in the ILC lineage. 3 days later, these mice were infected with 500 L3 N brasiliensis. Lung tissues were collected and analyzed 5 days after worm infection. (a) Intracellular staining of IL-5 and IL-13 in lung ILC2s. (b, c) Frequency and absolute account of IL-5+ ILC2s in lung. (d, e) Frequency and absolute account of IL-13+ ILC2s in lung. (f) Total number of lung ILC2s. ( $n=7$ for black group and $n=8$ for red group in $b-f)(\mathbf{g})$ Flow cytometric analysis of eosinophils (CD45+Ly6G ${ }^{-} C^{2} 11 b^{+}$SiglecF ${ }^{+}$) in lung. Cells were gated on CD45+'Ly6G- live single cells. (h) Absolute count of lung eosinophils. (i) Worm burden in the small intestine collected on day 7 post infection. ( $n=8$ for black group and $n=9$ for red group in $\mathbf{h}-\mathbf{i}$ ) Each symbol represents an individual mouse ( $\mathbf{b}-\mathbf{f}, \mathbf{h}$, and $\mathbf{i}$ ). Data are representative of 3 independent experiments and are presented as mean \pm SEM. Two-tailed unpaired Student's $t$ test. 
a
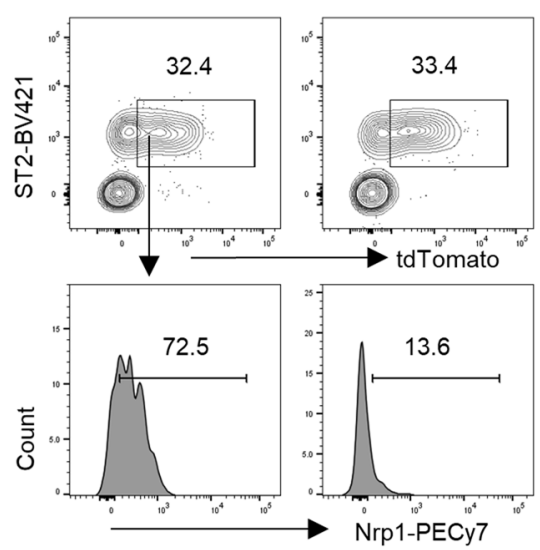

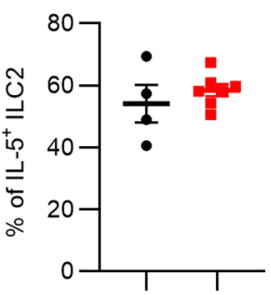

d

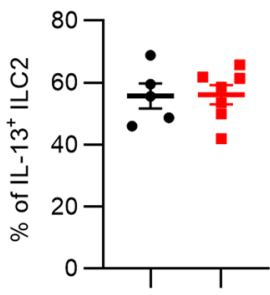

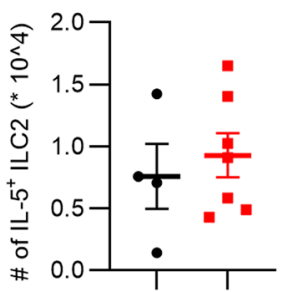

$\mathrm{e}$

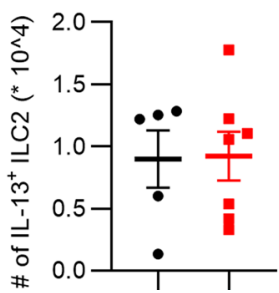

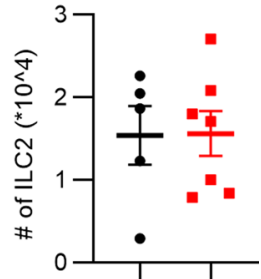

- $\mathrm{R} 5 /+$

- $\mathrm{R} 5 /+N r p 1^{f / f}$

Extended Data Fig. 4 | Nrp1 deficiency does not affect ILC2 compartment in R5/ + Nrp 1/f $^{\text {mice }}$ under steady state. (a)The expression of Nrp1 on lung ILC2s in R5/ + and R5/ + Nrp1//f mice was analyzed by flow cytometry. Cells was gated on lin-CD45 + CD127 + lymphocytes. (b, c) Frequency and absolute number of IL-5+ILC2s in lung ( $n=4$ for black group and $n=7$ for red group). (d, e) Frequency and absolute number of IL-13+ILC2s in lung. (f) Absolute count of total ILC2s in lung. ( $n=5$ for black group and $n=7$ for red group in $\mathbf{d - f}$ ) Each symbol represents an individual mouse (b-f). Data are representative of 3 independent experiments and are presented as mean \pm SEM. 

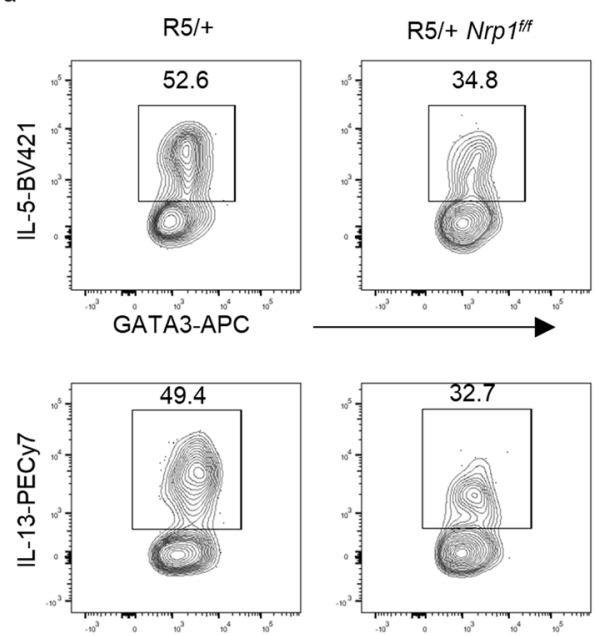

GATA3-APC

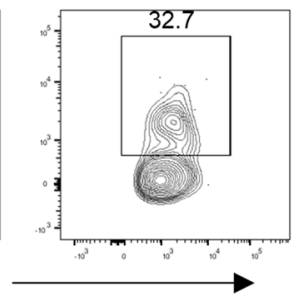

b
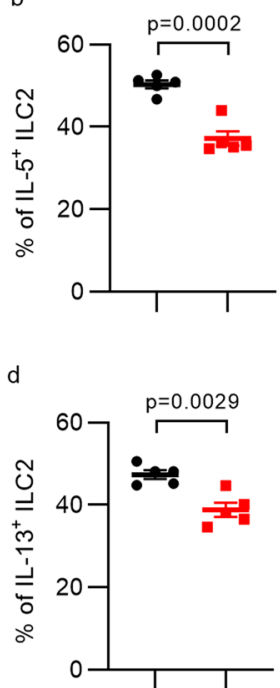

c

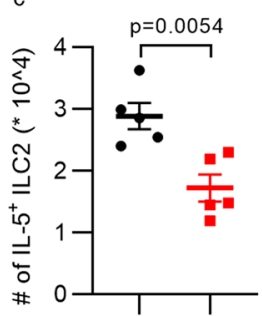

e

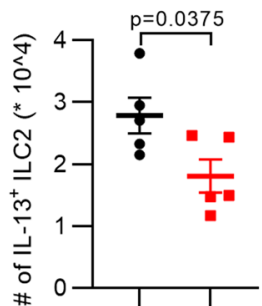

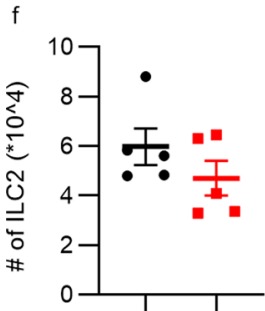

- $\mathrm{R} 5 /+$

- R5/+Nrp1 $1^{\text {f/i }}$
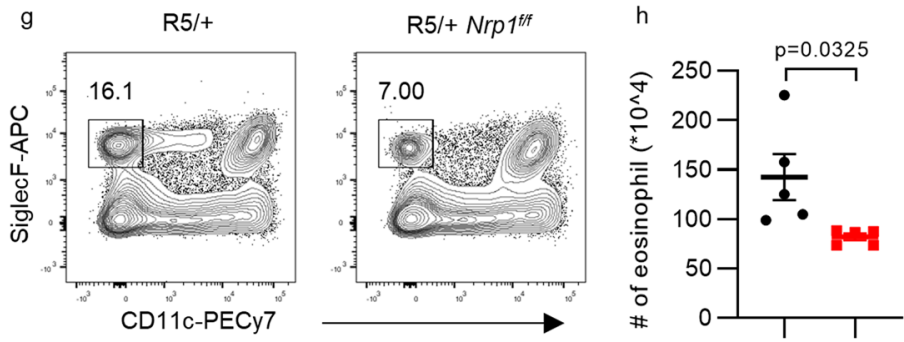

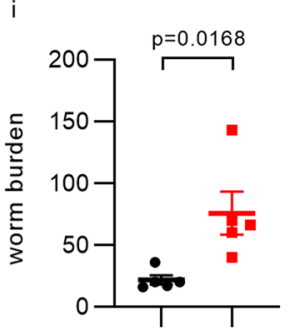

- $\mathrm{R} 5 /+$

- $\mathrm{R} 5 /+\mathrm{Nrp} 1^{\mathrm{f} / \mathrm{f}}$

Extended Data Fig. 5 | Loss of Nrp1 reduces ILC2 function and anti-helminth immunity in R5/ + Nrp1/f/f mice. a-i R5/ + and R5/ + Nrp ${ }^{1 / f}$ mice were infected with 500 L3 N brasiliensis subcutaneously. Lung tissues were collected and analyzed 5 days after worm infection. (a) Intracellular staining of IL-5 and IL-13 in lung ILC2s. (b, c) Frequency and absolute account of IL- $5^{+}$ILC2s in lung ( $n=5 /$ group). (d, e) Frequency and absolute account of IL-13+ ILC2s in lung ( $n=5 /$ group). (f) Total number of lung ILC2s $(n=5 / g r o u p)$. (g) Flow cytometric analysis of eosinophils in lung. Cells were gated on CD45+ CD11b+Ly6G- live single cells. (h) Absolute count of lung eosinophils ( $n=5 / g r o u p)$. (i) Worm burden in the small intestine collected on day 7 post infection ( $n=5 /$ group). Each symbol represents an individual mouse ( $\mathbf{b}-\mathbf{f}, \mathbf{h}$, and $\mathbf{i})$. Data are representative of 3 independent experiments and are presented as mean \pm SEM. Two-tailed unpaired Student's t test. 
PBS

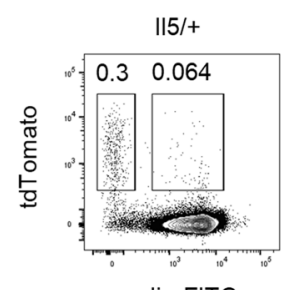

lin-FITC
$115 /+N r p 1^{f / f}$
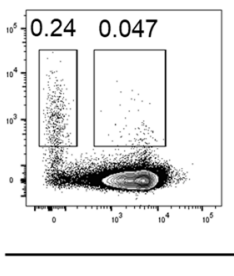

BLM

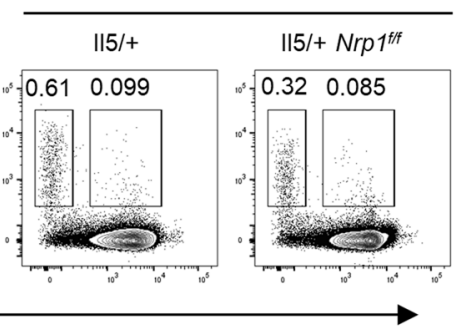

b

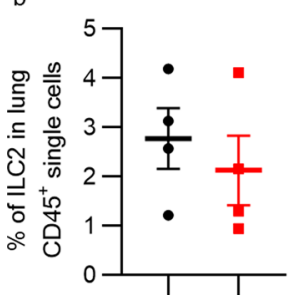

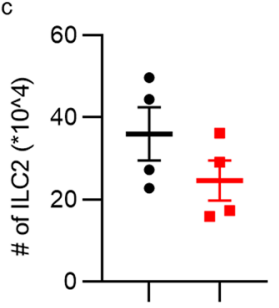

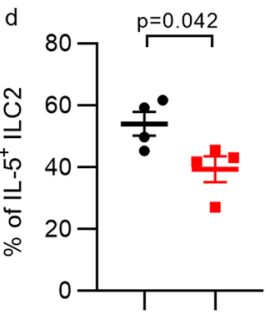

- Rag2 $\% / 12$ rg $\%+$ WTILC2s

- Rag $^{-\kappa} / 12 \mathrm{rg}^{--}+\mathrm{KO}$ ILC2s

Extended Data Fig. 6 | Nrp1 deficiency leads to reduced ILC2 function during pulmonary fibrosis. (a) R5/ + and R5/ + Nrp1//f mice were challenged with bleomycin to induce pulmonary fibrosis. Mice were sacrificed on day 21 and lung tissues were collected. Flow cytometric analysis of IL-5+ lymphocytes. Cells were gated on live CD45+ lymphocytes. b-e ILC2s isolated from lung and large intestine of R5/ + or R5/ + Nrp $1^{\text {t/f }}$ mice were transferred intravenously

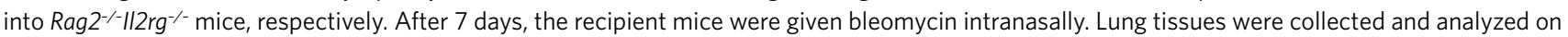
day 21 of fibrosis. (b, c) Frequency and absolute number of lung ILC2s $(n=4 / g r o u p)$. (d, e) Frequency and absolute number of IL-5+ ILC2s in lung ( $n=4 /$ group). Each symbol represents an individual mouse (b-e). Data are representative of 2-3 independent experiments and are presented as mean \pm SEM. Two-tailed unpaired Student's t test. 
DMSO

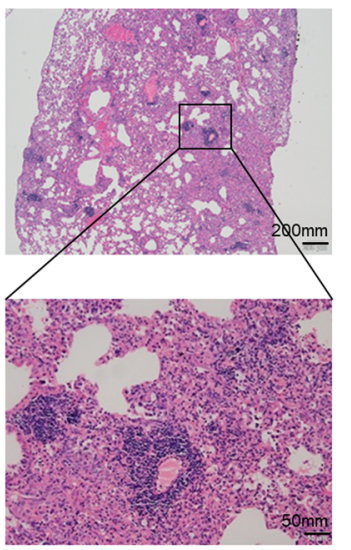

EG00229
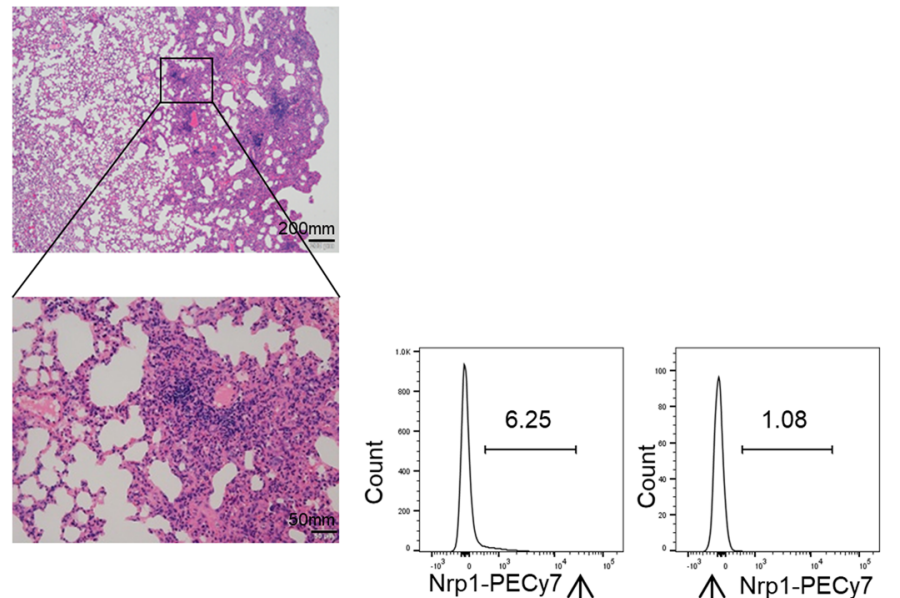

b
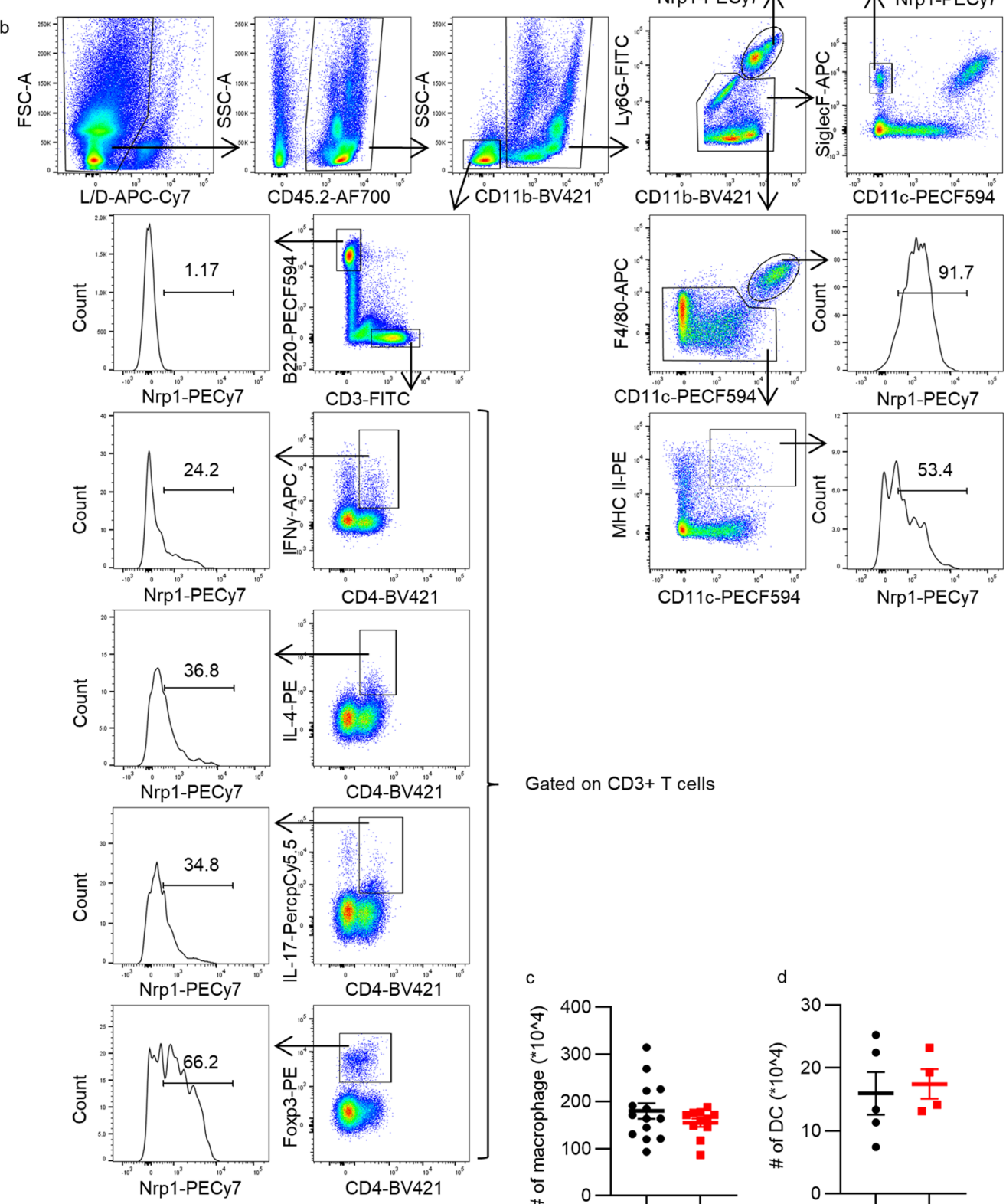

Gated on CD3+ T cells
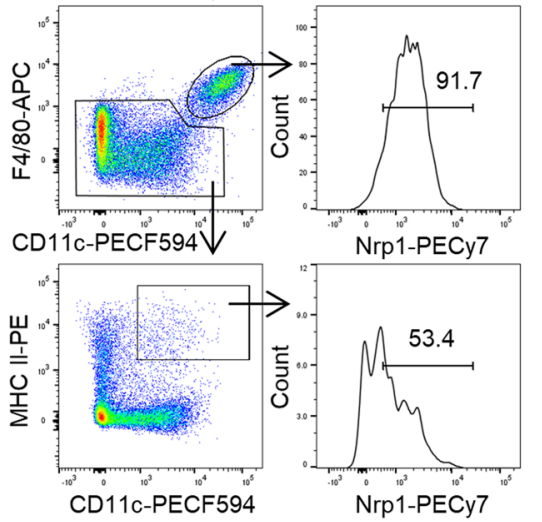

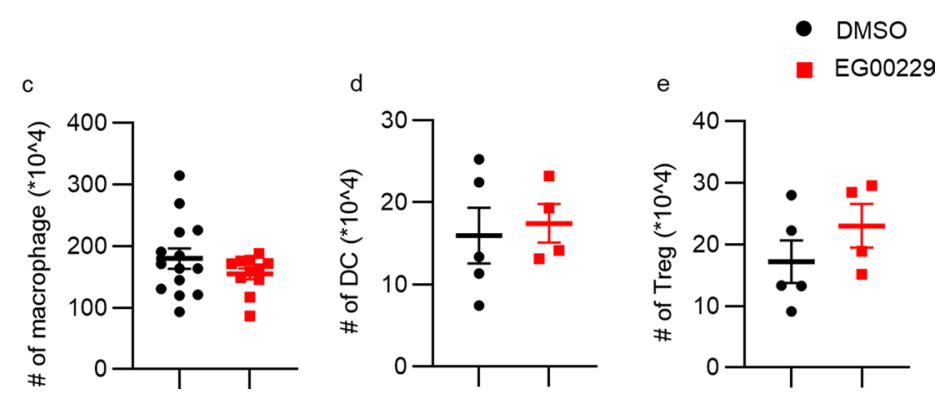

Extended Data Fig. 7 | See next page for caption. 
Extended Data Fig. 7 | The expression profile of Nrp1 in lung immune cells. a, c-e Wild-type mice were challenged with bleomycin (2 mg/kg body weight) intranasally to induce fibrosis, and subsequently were treated with EG00229 or DMSO control for three weeks. (a) H\&E staining of the representative lung tissue. (b) Nrp1 expression in lung immune cells from WT mice was analyzed by flow cytometry. (c-e) Absolute numbers of lung macrophages ( $\mathrm{n}=14$ for DMSO, $n=11$ for EG00229 in c), DCs and Treg cells ( $n=5$ for DMSO, $n=4$ for EG00229 in d-e) in each group of mice. Each symbol represents an individual mouse (c-e). Data are representative of at least 3 independent experiments and are presented as mean \pm SEM. 
a
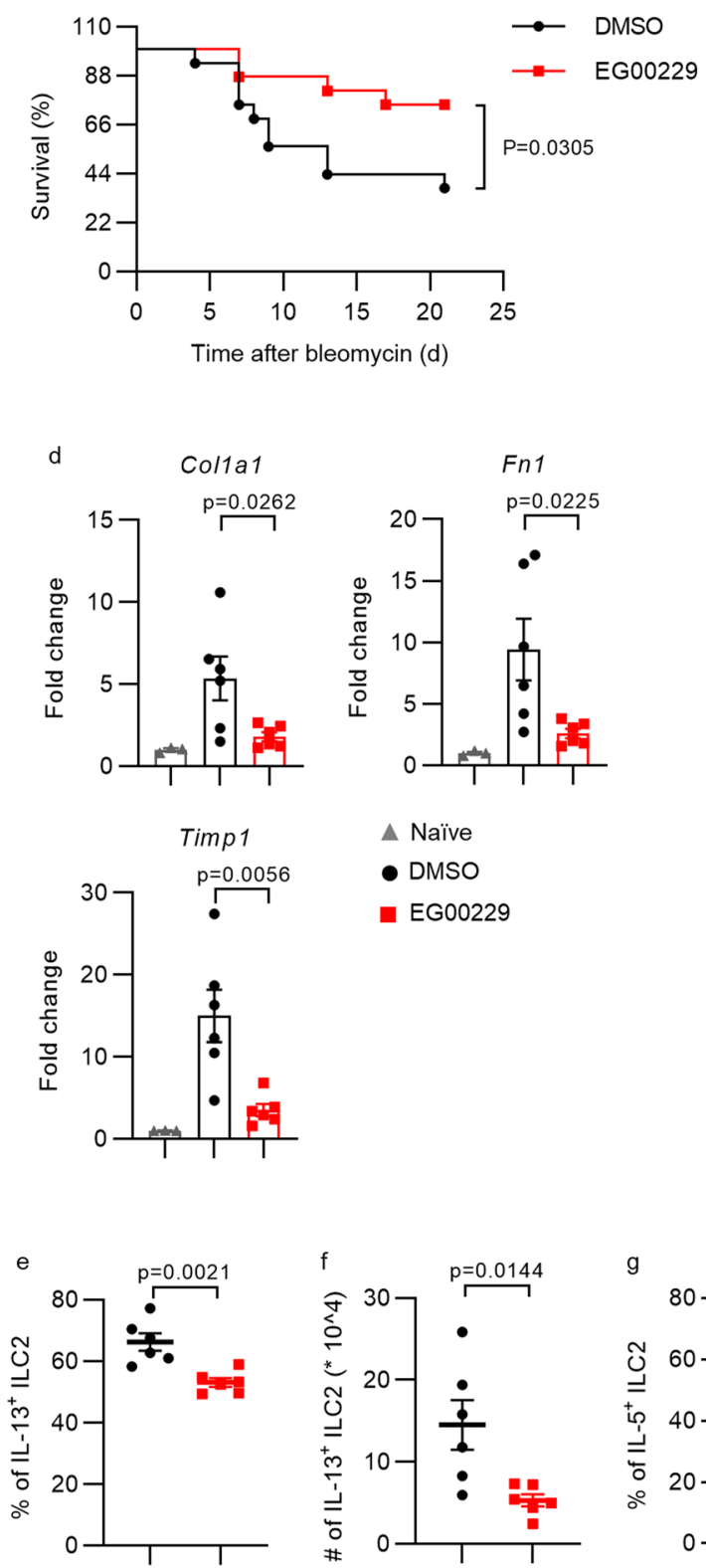
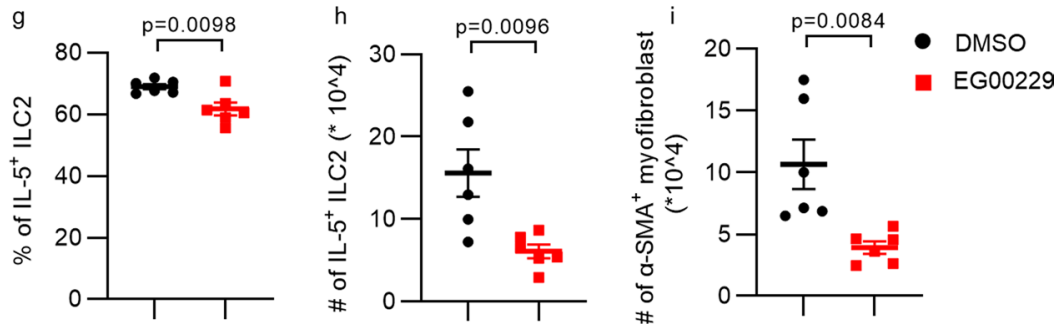

Extended Data Fig. 8 | Treatment with EG00229 intranasally alleviates pulmonary fibrosis. a-i Bleomycin-challenged mice were treated with EG00229 $(2.5 \mathrm{mg} / \mathrm{kg}$ body weight in $20 \mu \mathrm{l}$ PBS) or DMSO control intranasally three times weekly for three weeks. (a) The survival rate of mice treated with EG00229 or DMSO control was observed for a period of 21 days ( $n=16 /$ group). (b, c) H\&E staining and Masson's trichrome staining of lung tissues. (d) mRNA expression of the fibrotic genes in lung ( $n=3,6,6$ for PBS, DMSO, and EG00229 group). (e, f) Frequency and absolute count of IL-13+ ILC2s ( $n=6 /$ group). ( $(\mathbf{g}, \mathbf{h})$ Frequency and absolute count of IL-5+ ILC2s ( $n=6 /$ group). (i) Total number of $\alpha$-SMA+ myofibroblast $(n=6 / g r o u p)$. Each symbol represents an individual mouse (d-i). Data are representative of at least 3 independent experiments and are presented as mean \pm SEM. (a) Log-rank test. (d-i) Two-tailed unpaired Student's t test. 
$\lg G 1+D M S O$

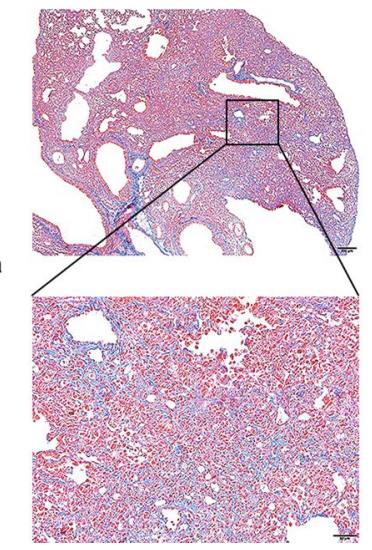

$\lg 1+E G 00229$

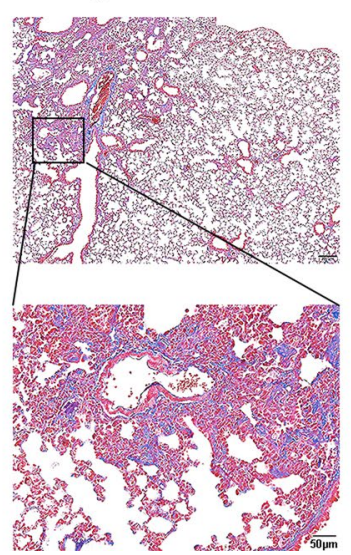

aThy1.2+DMSO

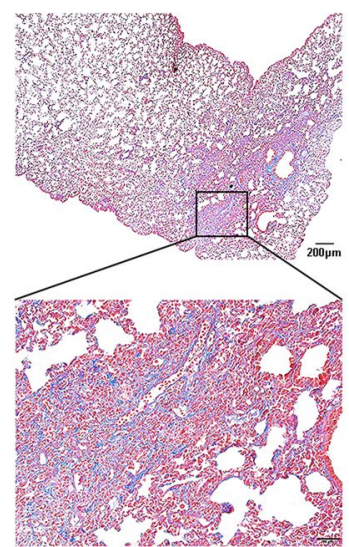

aThy1.2+EG00229

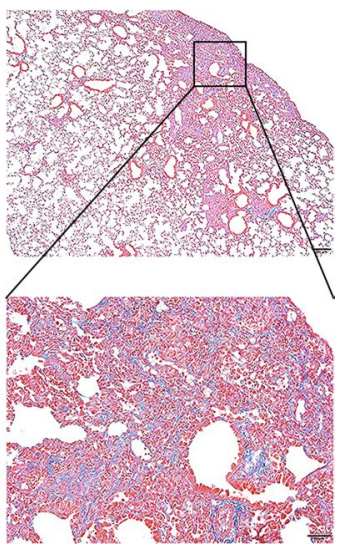

Extended Data Fig. 9 | The therapeutic effect of EG00229 on pulmonary fibrosis is dependent on lung ILC2s. (a) Bleomycin-challenged Rag1 ${ }^{1 /-}$ mice were treated with EG00229 (10 mg/kg body weight) or DMSO control intraperitoneally for three weeks. Each group of mice were also received 200 $\mu \mathrm{g}$ of anti-Thy1.2 or isotype control antibodies every 3 days throughout the treatment. Masson's trichrome staining for each group was shown. Data are representative of 3 independent experiments. 


\section{Reporting Summary}

Nature Portfolio wishes to improve the reproducibility of the work that we publish. This form provides structure for consistency and transparency in reporting. For further information on Nature Portfolio policies, see our Editorial Policies and the Editorial Policy Checklist.

\section{Statistics}

For all statistical analyses, confirm that the following items are present in the figure legend, table legend, main text, or Methods section.

$\mathrm{n} / \mathrm{a} \mid$ Confirmed

$\bigotimes$ The exact sample size $(n)$ for each experimental group/condition, given as a discrete number and unit of measurement

\ A statement on whether measurements were taken from distinct samples or whether the same sample was measured repeatedly

The statistical test(s) used AND whether they are one- or two-sided

Only common tests should be described solely by name; describe more complex techniques in the Methods section.

Х $\square$ A description of all covariates tested

\A description of any assumptions or corrections, such as tests of normality and adjustment for multiple comparisons

$\triangle$ A full description of the statistical parameters including central tendency (e.g. means) or other basic estimates (e.g. regression coefficient)

AND variation (e.g. standard deviation) or associated estimates of uncertainty (e.g. confidence intervals)

For null hypothesis testing, the test statistic (e.g. $F, t, r$ ) with confidence intervals, effect sizes, degrees of freedom and $P$ value noted Give $P$ values as exact values whenever suitable.

Х $\square$ For Bayesian analysis, information on the choice of priors and Markov chain Monte Carlo settings

Х $\square$ For hierarchical and complex designs, identification of the appropriate level for tests and full reporting of outcomes

$\bigotimes \square$ Estimates of effect sizes (e.g. Cohen's $d$, Pearson's $r$ ), indicating how they were calculated

\section{Our web collection on statistics for biologists contains articles on many of the points above.}

\section{Software and code}

Policy information about availability of computer code

Data collection Flow cytometry data were acquired with BD FACSDiva Software v8.0.2., qRT-PCR data were acquired with QuantStudio Real-Time PCR Software v1.3, RNA sequencing data were acquired with Illumina Hiseq X Ten system, histologic images were acquired using OLYMPUS BX53 system and cellSens Standard software.

Data analysis Flow cytometry data were analyzed with FlowJo Version 10. The parameters in t-SNE analysis included: FJComp-APC-PD1, FJComp-AF700CD45.2, FJComp-FITC- fixable viability stain, FJComp-PerCP efluor-CCR9, FJComp-BB790-CD69, FJComp-BUV395-GATA3, FJComp-BUV661CD25, FJComp-PE-KLRG1, FJComp-PECy5-CD127, FJComp-PECy7-Nrp1, FJComp-PECF594-Lin, FJComp-BV421-ST2, FJComp-BV605-IL-17RB, FJComp-BV650-SCA1, FJComp-BV750-MHC II, Iterations-1000, Perplexity-20, Eta(learning rate)-200. Statistical analyses were performed by Graphpad Prism 8.

The raw reads in RNA-seq were aligned to the mouse reference genome (version mm10) by using Hisat2 RNASeq alignment software. RNASeq differential expression analysis was performed by DESeq 2.

Heatmap was generated with software Heml 1.0.

For manuscripts utilizing custom algorithms or software that are central to the research but not yet described in published literature, software must be made available to editors and

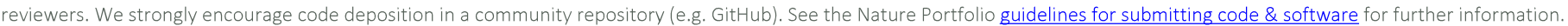


Policy information about availability of data

All manuscripts must include a data availability statement. This statement should provide the following information, where applicable:

- Accession codes, unique identifiers, or web links for publicly available datasets

- A description of any restrictions on data availability

- For clinical datasets or third party data, please ensure that the statement adheres to our policy

The accession number for the RNA-sequencing data files reported in this paper is: GSE168809. ILC2 sCRNA-seq dataset published in previous study was GSE117568. ILC2 bulk RNA-seq dataset published in previous study was GSE126107. The raw reads of RNA-seq were aligned to the mouse reference genome (version mm10).

\section{Field-specific reporting}

Please select the one below that is the best fit for your research. If you are not sure, read the appropriate sections before making your selection.

$\bigotimes$ Life sciences $\quad \square$ Behavioural \& social sciences $\quad \square$ Ecological, evolutionary \& environmental sciences

For a reference copy of the document with all sections, see nature.com/documents/nr-reporting-summary-flat.pdf

\section{Life sciences study design}

All studies must disclose on these points even when the disclosure is negative.

Sample size $\quad$ Sample size was chosen based on prior knowledge from previous experiments. Key experiments were repeated by 2 independent researchers. The lung tissues from at least 3 animals per genotype in this study were analyzed to ensure that differences were reproducible. Variability in the ex vivo and in vitro assays used in this study tends to be low, so $n>=3$ is the accepted norm in the field.

Data exclusions No data were excluded in this study.

Replication Both in vitro and in vivo experiments were performed with at least 3 biological replicates.

Randomization Littermate mice were randomly grouped into control and treatment groups for all experiments in this study.

Blinding $\quad$ For quantifications that were done with FACS, blinding was performed by labeling the test tubes numerically without prior knowledge of the genotype/treatment of the sample. The other samples were not blinded to the authors, since all measurements were objective and the conclusions were based on multiple independent experiments, technical replicates and statistical significance.

\section{Reporting for specific materials, systems and methods}

We require information from authors about some types of materials, experimental systems and methods used in many studies. Here, indicate whether each material, system or method listed is relevant to your study. If you are not sure if a list item applies to your research, read the appropriate section before selecting a response.

\begin{tabular}{l|l} 
Materials \& experimental systems \\
\hline$n / a$ & Involved in the study \\
\hline & $\bigotimes$ Antibodies \\
$\searrow$ & $\square$ Eukaryotic cell lines \\
$\square$ & $\square$ Palaeontology and archaeology \\
$\square$ & $\bigotimes$ Animals and other organisms \\
$\square$ & $\bigotimes$ Human research participants \\
$\searrow$ & $\square$ Clinical data \\
$\searrow$ & $\square$ Dual use research of concern
\end{tabular}

\begin{tabular}{l|l} 
Methods \\
\hline n/a & Involved in the study \\
$\square$ & $\square$ ChIP-seq \\
$\square$ & $\bigotimes$ Flow cytometry \\
$\searrow$ & $\square$ MRI-based neuroimaging
\end{tabular}

\section{Antibodies}

Antibodies used

FACSymphonyA3 flow-cytometric panel:

Fixable Viability Stain 520 BD Biosciences 564407 (1:1000)

CD199 (CCR9) (eBioCW-1.2 (CW-1.2)), PerCP-eFluor 710 Termo Fisher Scientific 46-1991-82 (1:200)

BB790-P Hamster Anti-Mouse CD69 BD Biosciences 624296 (1:200)

Brilliant Violet 421 anti-mouse IL-33R $\alpha$ (IL1RL1, ST2) Biolegend 145309 (1:200)

BV605 Rat Anti-Mouse IL-17 Receptor B BD Biosciences 743817 (1:200)

BV650 Rat Anti-Mouse Ly-6A/E (SCA1) BD Biosciences 744325 (1:200)

BV750 Rat Anti-Mouse I-A/I-E (MHC II) BD Biosciences 624380 (1:200) 
BUV395 Mouse Anti-GATA3 BD Biosciences 565448 (1:100)

BUV661 Rat Anti-Mouse CD25 BD Biosciences 624285 (1:200)

APC Hamster Anti-Mouse CD279 (PD-1) BD Biosciences 562671 (1:200)

Alexa Fluor 700 anti-mouse CD45.2 Biolegend 109822 (1:200)

PE Hamster Anti-Mouse KLRG1 BD Biosciences 561621 (1:200)

PE-CF594 Hamster Anti-Mouse CD3e BD Biosciences 562286 (1:200)

PE-CF594 Rat Anti-Mouse CD45R BD Biosciences 562290 (1:200)

PE-CF594 Rat Anti-Mouse Ly-6G and Ly-6C BD Biosciences 562710 (1:200)

PE-CF594 Rat Anti-Mouse CD11b BD Biosciences 562287 (1:200)

PE-CF594 Hamster Anti-Mouse CD11c BD Biosciences 562454 (1:200)

PE-CF594 Rat Anti-Mouse CD5 BD Biosciences 624352 (1:200)

PE/Cyanine5 anti-mouse CD127 (IL-7Ra) Antibody Biolegend 135015 (1:200)

CD304 (Neuropilin-1) Monoclonal Antibody (3DS304M), PE-Cyanine7 Termo Fisher Scientific 25-3041-80 (1:200)

Mouse flow cytometry antibodies:

TruStain FCX (anti-mouse CD16/32) Antibody Biolegend 101320 (1:100)

Fixable Viability Stain 620 BD Biosciences 564996 (1:1000)

LIVE/DEAD ${ }^{\text {TM }}$ Fixable Near-IR Dead Cell Stain Kit TermoFisher Scientific L10119 (1:1000)

Anti-Mouse CD3e FITC (145-2C11) TermoFisher Scientific 11-0031-85 (1:200)

Anti-Mouse CD11b FITC (M1/70) TermoFisher Scientific 11-0112-81 (1:200)

Anti-Mouse CD11c FITC (N418) TermoFisher Scientific 11-0114-81 (1:200)

Anti-Mouse CD45R (B220) FITC (RA3-6B2) TermoFisher Scientific 11-0452-82 (1:200)

Anti-Mouse CD45.1 FITC (clone A20) Biolegend 110706 (1:200)

Alexa Fluor ${ }^{\circledast} 647$ Rat Anti-Mouse Siglec-F BD Biosciences 562680 (1:200)

FITC Rat anti-Mouse Ly-6G BD Biosciences 551460 (1:200)

PE Rat Anti-Mouse I-A/I-E BD Biosciences 562010 (1:200)

APC Rat Anti-Mouse F4/80 BD Biosciences 566787 (1:200)

BV421 Rat Anti-CD11b BD Biosciences 562605 (1:200)

APC Rat Anti-Mouse IFN- $\gamma$ BD Biosciences 554413 (1:100)

PE Rat Anti-Mouse IL-4 BD Biosciences 562044 (1:100)

Pacific Blue ${ }^{\mathrm{TM}}$ Rat Anti-Mouse CD4 BD Biosciences 558107 (1:200)

PE Rat anti-Mouse Foxp3 BD Biosciences 560408 (1:100)

PE/Cy7 anti-mouse CD45 Biolegend 103113 (1:200)

Brilliant Violet 421 anti-mouse CD127 (IL-7R $\alpha$ ) Biolegend 135027 (1:200)

FITC anti-mouse CD31 Biolegend 102405 (1:200)

APC anti-mouse CD326 (Ep-CAM) Biolegend 118213 (1:100)

Brilliant Violet 421 Goat anti-mouse IgG (minimal x-reactivity) Antibody Biolegend 405317 (1:1000)

Brilliant Violet 421 anti-mouse/human IL-5 Antibody Biolegend 504311 (1:100)

PE Mouse anti-Smad2 (pS465/pS467)/Smad3 (pS423/pS425) BD Biosciences 562586 (1:50)

CD25 Monoclonal Antibody (PC61.5), APC Termo Fisher Scientific 17-0251-82 (1:200)

Gata-3 Monoclonal Antibody (TWAJ), eFluor 660 Termo Fisher Scientific 50-9966-42 (1:100)

IL-13 Monoclonal Antibody (eBio13A), PE-Cyanine7 Termo Fisher Scientific 25-7133-80 (1:100)

IL-17A Monoclonal Antibody (eBio17B7), PerCP-Cyanine5.5 Termo Fisher Scientific 45-7177-80 (1:100)

CD90.2 (Thy-1.2) Monoclonal Antibody (53-2.1), PE-Cyanine7 Termo Fisher Scientific 25-0902-82 (1:200)

Human flow cytometry antibodies:

Human TruStain FcX (Fc Receptor Blocking Solution) Biolegend 422302 (1:100)

FITC anti-human CD3 Biolegend 300452 (1:100)

FITC anti-human CD5 Biolegend 300606 (1:100)

FITC anti-human CD14 Biolegend 301804 (1:100)

FITC anti-human CD19 Biolegend 302256 (1:100)

CD1a Monoclonal Antibody (HI149), FITC Termo Fisher Scientific 11-0019-41 (1:100)

CD11b (activation epitope) Monoclonal Antibody (CBRM1/5), FITC Termo Fisher Scientific 11-0113-42 (1:100)

CD11c Monoclonal Antibody (3.9), FITC Termo Fisher Scientific 11-0116-42 (1:100)

CD15 Monoclonal Antibody (HI98), FITC Termo Fisher Scientific 11-0159-42 (1:100)

CD16 Monoclonal Antibody (eBioCB16 (CB16)), FITC Termo Fisher Scientific 11-0168-42 (1:100)

FceR1 alpha Monoclonal Antibody (AER-37 (CRA1)), FITC Termo Fisher Scientific 11-5899-42 (1:100)

CD127 Monoclonal Antibody (eBioRDR5), PE Termo Fisher Scientific 12-1278-42 (1:100)

Alexa Fluor 700 anti-human CD45 Biolegend 304024 (1:100)

Brilliant Violet 421 anti-human CD117 (c-kit) Biolegend 313216 (1:100)

PE/Dazzle ${ }^{\text {TM }} 594$ anti-human CD294 (CRTH2) Antibody Biolegend 350125 (1:50)

Alexa Fluor ${ }^{\circledR} 647$ Mouse Anti-Human Neuropilin-1 (CD304) BD Biosciences 566048 (1:100)

WB antibodies:

Human/Mouse/Rat alpha-Smooth Muscle Actin Antibody R\&D systems MAB1420-SP (1:100 for flow, 1 : 2500 for WB)

GAPDH (14C10) Rabbit mAb \#2118 Cell Signaling Technology 2118S (1:1000)

Anti-mouse IgG, HRP-linked Antibody \#7076 Cell Signaling Technology 7076S (1:3000)

Anti-rabbit IgG, HRP-linked Antibody \#7074 Cell Signaling Technology 7074S (1:3000)

Validation

Fixable Viability Stain 520 BD Biosciences Cat\#564407 (1:1000)

(App: FC React: Mammalian cells)

PerCP-eFluor 710 Mouse Anti-Mouse CD199 (CCR9) (Clone eBioCW-1.2 (CW-1.2)) Thermo Fisher Scientific Cat\#46-1991-82 (1:200)

(App: FC React: Mouse)

BB790-P Hamster Anti-Mouse CD69 (Clone H1.2F3) BD Biosciences Cat\#624296 (1:200) 


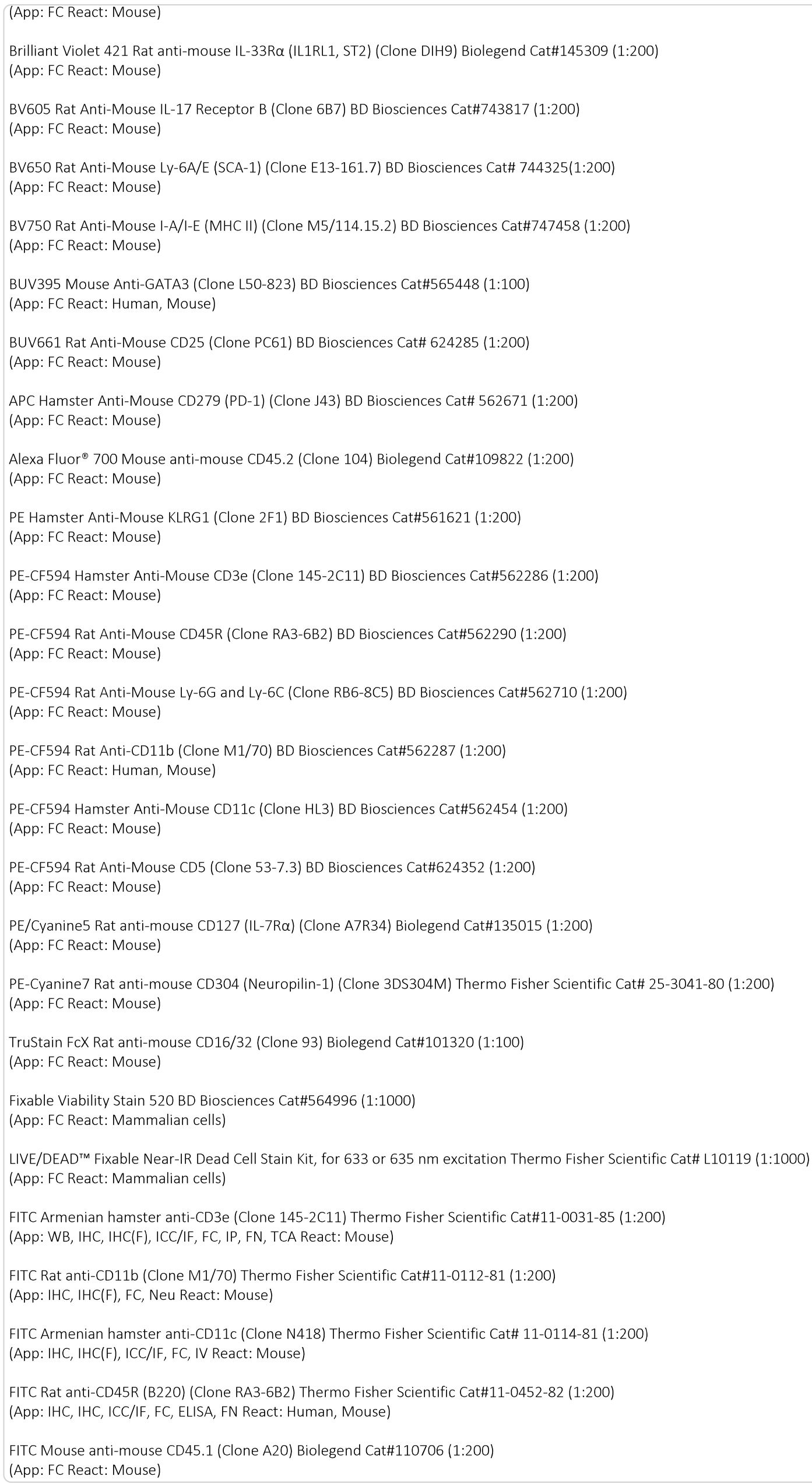




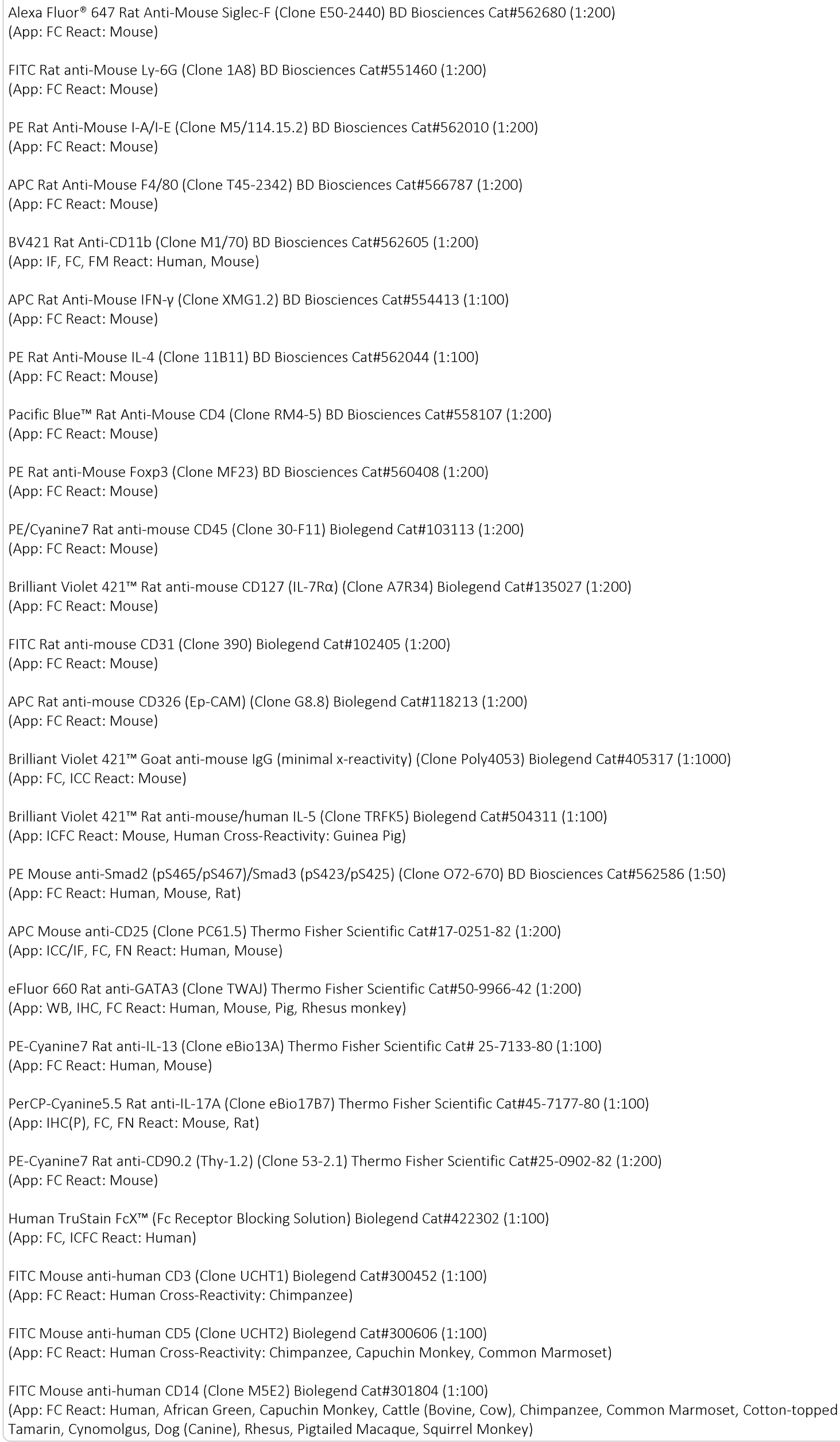


FITC Mouse anti-human CD19 (Clone HIB19) Biolegend Cat\#302256 (1:100)

(App: FC React: Human, Chimpanzee, Rhesus)

FITC Mouse anti-human CD1a (Clone HI149) Thermo Fisher Scientific Cat\#11-0019-41 (1:100)

(App: FC React: Human)

FITC Mouse anti-human CD11b (activation epitope) (Clone CBRM1/5) Thermo Fisher Scientific Cat\#11-0113-42 (1:100)

(App: FC React: Human)

FITC Mouse anti-human CD11c (Clone 3.9) Thermo Fisher Scientific Cat\#11-0116-42(1:100)

(App: FC React: Human)

FITC Mouse anti-human CD15 (Clone HI98) Thermo Fisher Scientific Cat\#11-0159-42 (1:100)

(App: WB, IHC, ICC/IF, FC React: Human)

FITC Mouse anti-human CD16 (Clone eBioCB16 (CB16)) Thermo Fisher Scientific Cat\#11-0168-42 (1:100)

(App: ICC/IF, FC, TCA React: Human)

FITC Mouse anti-human FceR1 alpha (Clone AER-37 (CRA1)) Thermo Fisher Scientific Cat\# 11-5899-42 (1:100)

(App: FC React: Human)

PE Mouse anti-human CD127 (Clone eBioRDR5) Thermo Fisher Scientific Cat\# 12-1278-42 (1:100)

(App: IHC(F), FC React: Human)

Alexa Fluor ${ }^{\circledast} 700$ Mouse anti-human CD45 (Clone HI30) Biolegend Cat\# 304024 (1:100)

(App: FC React: Human)

Brilliant Violet 421 $1^{\text {TM }}$ Mouse anti-human CD117 (c-kit) (Clone 104D2) Biolegend Cat\# 313216 (1:100)

(App: FC React: Human Cross-Reactivity: Cynomolgus, Cattle (Bovine, Cow))

PE/Dazzle ${ }^{\text {TM }} 594$ Rat anti-human CD294 (CRTH2) (Clone BM16) Biolegend Cat\# 350125 (1:100)

(App: FC React: Human)

Alexa Fluor ${ }^{\circledast} 647$ Mouse Anti-Human Neuropilin-1 (CD304) (Clone U21-1283) BD Biosciences Cat\# 566048 (1:100)

(App: FC React: Human)

Mouse anti-Human/Mouse/Rat alpha -Smooth Muscle Actin (Clone 1A4) R\&D systems Cat\# MAB1420-SP (1:100 for flow, 1: 2500 for WB)

(App: FC, WB, SM, IHC, CyTOF, ISH-IHC, ICC React: Human, Mouse, Rat)

Rabbit-GAPDH (Clone 14C10) Cell Signaling Technology Cat\#2118S (1:1000)

(App: WB, IHC, IF, F React: Human, Mouse, Rat, Monkey, Bovine, Pig)

HRP-linked Horse Anti-mouse IgG Cell Signaling Technology Cat\#7076S (1:30000)

(App: WB React: Mouse)

HRP-linked Goat Anti-rabbit IgG Cell Signaling Technology Cat\#7074S (1:3000)

(App: WB React: Rabbit)

\section{Animals and other organisms}

Policy information about studies involving animals; ARRIVE guidelines recommended for reporting animal research

Laboratory animals

Wild-type mice, including C57BL/6, BALB/C, DBA, ICR, and S129 backgrounds were purchased from Shanghai SLAC Laboratory Animal Co.Ltd. CD45.1 mice, Rag1-/- mice, Id2cre-ERT2 mice, II5tm1.1(icre)Lky/J(R5/+) mice and Nrp1tm2Ddg/J(Nrp1f/f) mice were purchased from the Jackson laboratory (Bar Harbor, Me). Rag2-/-II rg-/- mice were purchased from Taconic Biosciences (Rensselaer, NY). All mice were bred and maintained at accredited animal facilities under specific pathogen-free conditions in individually ventilated cages on a strict 12-h day-night cycle with a regular chow diet. Mice used in this study were 6-8 weeks old and sex-matched unless otherwise indicated in the text.

\begin{tabular}{l|l} 
Wild animals $\quad$ The study did not involve wild animals. \\
\hline
\end{tabular}

Field-collected samples The study did not involve samples collected from the field.

Ethics oversight $\quad$ All animal experiments were performed according to local guidelines for the use and care of laboratory animals as provided by Shanghai Jiao Tong University School of Medicine Institutional Animal Care and Use Committees (IACUC)

Note that full information on the approval of the study protocol must also be provided in the manuscript.

\section{Human research participants}

Policy information about studies involving human research participants

Population characteristics

Human blood samples were collected from both female and male healthy donors, with age ranging from 25 to 40 (ethics 
Flow cytometry analysis:

Samples were collected from patients with non-small cell lung cancer (NSCLC) or NSCLC with idiopathic pulmonary fibrosis (IPF). Bronchoalveolar lavage fluid and tumor adjacent lung tissues were retrieved through Shanghai Chest Hospital following the study protocol approved by KS(Y)21172. Informed consent was obtained from all patients. The study was performed in strict compliance with all institutional ethical regulations. Patients' characteristics are detailed in Supplementary Table 1.

Gender: Female $n=7$ (54\%), Male $n=6(46 \%)$

Age at diagnosis: $<60 n=5(38 \%),>60 n=8(62 \%)$

Pathological diagnosis: la $n=3(23 \%)$, la with IPF $n=1(8 \%)$, lb $n=3(23 \%)$, lb with IPF $n=1(8 \%)$, Ila $n=4$ (30\%), lla with IPF $n=1$ $(8 \%)$

Treatment: Vadio assisted thoracic surgery (VATS) pulmonary lobectomy $n=11(85 \%)$, VATS pulmonary segmentectomy $n=2$ $(15 \%)$

Recruitment

Lung cancer patients eligible for surgical reception at Shanghai Chest Hospital were recruited to participate in an Institutional Reviews Board-approved protocol (ethics approval number: KS(Y)21172, Shanghai Chest Hospital). All patients who provided informed consent had samples collected; all study procedures were conducted in strict compliance with all ethical and institutional regulations.

Ethics oversight

KS(Y)21172

Note that full information on the approval of the study protocol must also be provided in the manuscript.

\section{Flow Cytometry}

Plots

Confirm that:

\The axis labels state the marker and fluorochrome used (e.g. CD4-FITC).

\The axis scales are clearly visible. Include numbers along axes only for bottom left plot of group (a 'group' is an analysis of identical markers).

\All plots are contour plots with outliers or pseudocolor plots.

\A numerical value for number of cells or percentage (with statistics) is provided.

\section{Methodology}

Sample preparation

Instrument

Software

Cell population abundance

Gating strategy

\Tick this box to confirm that a figure exemplifying the gating strategy is provided in the Supplementary Information.

\begin{abstract}
excluded by using Fixable Viability Stain 520. Immune cells were gated on CD45+ and then lymphoid and myeloid cells populations were identified as described in Figures or Figure legends. Positive and negative cells were identified based on clear boudaries between the two populations. For markers with no clear boundaries between positive and negative populatioins, FMO was used as a control.

Lung or pancrea was cut into pieces and digested in $5 \mathrm{ml}$ of RPMI 1640 medium (Thermo Fisher Scientific) containing DNase I $(750 \mathrm{~g} / \mathrm{ml}$ for digestion of lung, pancreas was digested without DNasel, Sigma-Aldrich) and collagenase VIII (200U/ml, SigmaAldrich) at $37^{\circ} \mathrm{C}$ for $1 \mathrm{~h}$ (for digestion of lung) or 30 minutes (for digestion of pancreas). The digested tissues were passed through a $70 \mu \mathrm{m}$ cell strainer after vigorous shaking. Mononuclear cells were then harvested from the interphase of a $40 \%$ and $80 \%$ Percoll (GE Healthcare) gradient after a spin at 2500 rpm for 20 minutes at room temperature. When fat tissues and payer's patches were removed, the large intestine and small intestine were cut into pieces and washed in PBS. Intestines were incubated with shaking at 220rpm in PBS containing $1 \mathrm{mM}$ dithiothreitol (Sigma) for 10 minutes and then in PBS containing 30mM ethylenediaminetetraacetic acid (EDTA, Sangon Biotech) at $37^{\circ} \mathrm{C}$ for 10 minutes for 2 cycles. Tissues were digested in RPMI 1640 medium containing DNase I $(150 \mu \mathrm{g} / \mathrm{ml})$ and collagenase VIII $(150 \mathrm{U} / \mathrm{ml})$ at $37^{\circ} \mathrm{C}$ in an incubator with $5 \% \mathrm{CO} 2$ for $1.5 \mathrm{~h}$. Intestinal mononuclear cells were harvested according to the protocols above. Bone marrow cells were processed by flushing femur and tibia by using a syringe. Red blood cells were removed with $3 \mathrm{ml}$ of ACK Lysing Buffer (Gibco) at $4^{\circ} \mathrm{C}$ for 3 minutes and centrifuged at $400 \mathrm{~g}$ for 5 minutes.

BD LSRFortessa ${ }^{\mathrm{TM}}$ X-20 Flow Cytometer; BD FACSymphony ${ }^{\mathrm{TM}}$ A3 Flow Cytometer; BD FACSAria ${ }^{\mathrm{TM}}$ III Cell Sorter

BD FACSDiva software was used for collecting flow data and Flowjo_V10 software was used for analyzing flow data.

About 0.2 million ILC2s were got from the lung from 5-10 mice, and the purity of the samples was about $98 \%$ as determined by flow cytometry.

In all experiments, populations were gated on FSC/SSC and single cells were retained using FSC-A/FSC-H. Dead cells were
excluded by using Fixable Viability Stain 520 . Immune cells were gated on CD45+ and then lymphoid and myeloid cells
populations were identified as described in Figures or Figure legends. Positive and negative cells were identified based on
clear boudaries between the two populations. For markers with no clear boundaries between positive and negative
populatioins, FMO was used as a control.
\end{abstract}

